\title{
ON A ROUGH PERTURBATION OF THE NAVIER-STOKES SYSTEM AND ITS VORTICITY FORMULATION
}

\author{
By Martina Hofmanová ${ }^{1}$, JAMES-MiChaEl LeAHY ${ }^{2}$ And TORSTEIN NilsSEN ${ }^{3}$ \\ ${ }^{1}$ Fakultät für Mathematik, Universität Bielefeld, hofmanova@math.uni-bielefeld.de \\ ${ }^{2}$ Department of Mathematics, Imperial College London, jleahy1@gmail.com \\ ${ }^{3}$ Institute of Mathematics, University of Agder, torstein.nilssen@uia.no
}

We introduce a rough perturbation of the Navier-Stokes system and justify its physical relevance from balance of momentum and conservation of circulation in the inviscid limit. We present a framework for a well-posedness analysis of the system. In particular, we define an intrinsic notion of strong solution based on ideas from the rough path theory and study the system in an equivalent vorticity formulation. In two space dimensions, we prove that well-posedness and enstrophy balance holds. Moreover, we derive rough path continuity of the equation, which yields a Wong-Zakai result for Brownian driving paths, and show that for a large class of driving signals, the system generates a continuous random dynamical system. In dimension three, the noise is not enstrophy balanced, and we establish the existence of local in time solutions.

\section{Introduction.}

1.1. General motivation. In this paper, we investigate well-posedness and stability of a rough-path perturbation of the Navier-Stokes system. The deterministic Navier-Stokes equations are a system of nonlinear partial differential equations that govern the velocity field $u$ and pressure $p$ of an incompressible homogeneous viscous fluid moving in some domain $\mathcal{D} \subseteq \mathbf{R}^{d}$ :

$$
\begin{aligned}
\partial_{t} u+(u \cdot \nabla) u & =-\nabla p+\vartheta \Delta u, \quad(t, x) \in(0, T) \times \mathcal{D}, \\
\nabla \cdot u & =0,\left.\quad u\right|_{t=0}=u_{0},
\end{aligned}
$$

where $\vartheta$ is the kinematic viscosity, $u_{0}$ is a given initial velocity and additional boundary conditions are needed depending on the domain $\mathcal{D}$. The system (1.1) can be derived from the basic physical principles by assuming conservation of mass and momentum in integral form, homogeneity, incompressibility (or conservation of kinetic energy) and viscous stress forces, and using Reynold's transport theorem. At least formally, the time-dependent vector field $u$ generates a time-homogeneous two-parameter flow $\eta_{s, t}$ on $\mathcal{D}$ :

$$
\dot{\eta}_{s, t}(x)=u_{t}\left(\eta_{s, t}(x)\right), \quad \eta_{s, s}(x)=x, \quad s \leq t, x \in \mathcal{D} .
$$

That is, a particle initially at a point $x \in \mathcal{D}$ at time $s$ moves to the point $\eta_{s, t}(x) \in \mathcal{D}$ at time $t$ in such way that at each $t^{\prime} \in(s, t)$, the instantaneous velocity is given by $u_{t^{\prime}}\left(\eta_{s, t^{\prime}}(x)\right)$.

In practice, solutions of the Navier-Stokes system are numerically approximated. Due to limited computational resources, there are usually subgrid scales that cannot be resolved by a direct numerical simulation. The nonlinear term $(u \cdot \nabla) u$ mixes the subgrid and grid scales. As such, accurate forecasts of turbulent fluid regimes are only possible at the moment if substantial computational resources are invested, which is not a luxury practitioners can 
afford in real-time applications where data is to be assimilated. Lewis Fry Richardson has said: "Big whirls have little whirls that feed on their velocity, and little whirls have lesser whirls and so on to viscosity." Here, Richardson is describing the direct energy cascade in 3D turbulence, in which energy is transferred from larger eddies (modes) to smaller eddies to the minimum scale at which the energy is dissipated by viscosity. In fluid dynamics and turbulence modeling especially, the search for tractable models for subgrid-scale dynamics that are closable, parameterizable, and preserve physical laws is ongoing (see, e.g., [26] for one such example). While all parameterization schemes are designed to improve the quality of forecasts, stochastic parameterization schemes have the additional advantage of providing a natural mechanism to quantify uncertainty in prediction.

An important property of a parameterized dynamical system is the stability of the dynamics with respect to the parameters. In order to define stability, one must specify a set of input parameters and an output set (of the dynamical system), and endow the corresponding sets with a topology. For a parameterized stochastic dynamical system, there are two main types of stability, which we will briefly explain. Let $S$ denote the output of a parameterized stochastic dynamical system, which takes values in a space $\mathcal{N}$ and depends on time $t \in \mathbf{R}_{+}$, space $x \in \mathcal{M}$, a set of parameters $\Theta$, and a sample space outcome $\omega \in \Omega$ (where $(\Omega, \mathcal{F}, \mathbf{P}$ ) is a probability space). Probabilistic stability usually means continuity of the map

$$
S: \Theta \rightarrow O \subset L^{0}\left(\Omega \times \mathbf{R}_{+} \times \mathcal{M} ; \mathcal{N}\right),
$$

where $O$ is a metric space contained in $L^{0}\left(\Omega \times \mathbf{R}_{+} \times \mathcal{M} ; \mathcal{N}\right)$, the space of measurable random variables from $\Omega \times \mathbf{R}_{+} \times \mathcal{M}$ to $\mathcal{N}$. Pathwise stability, on the other hand, means continuity of the map

$$
S: \Omega \times \Theta \rightarrow \tilde{O} \subset L^{0}\left(\mathbf{R}_{+} \times \mathcal{M} ; \mathcal{N}\right),
$$

where $\Omega$ shall be endowed with certain topology.

To study stability in this sense, a solution map needs to be constructed for each $\omega$; in other words, $S(\omega)$ is the outcome of a deterministic dynamical system. If $(\Omega, \mathcal{F}, \mathbf{P})$ is the canonical probability space for a multi-dimensional Wiener process and the model contains a stochastic integral, then, in general, there is no separable Banach space contained in the space of continuous functions $\Omega$ that contains the trajectories of the Wiener process almost-surely and for which the solution map $S$ is pathwise stable. The key idea of rough paths is to consider an enriched set $\boldsymbol{\Omega}$ of rough paths (i.e., an appropriate feature set for the Brownian paths) that contains additional information beyond the path itself, namely the iterated integrals of the path $\omega$, which one can construct by probabilistic methods. The map $S$ is then factorized as follows:

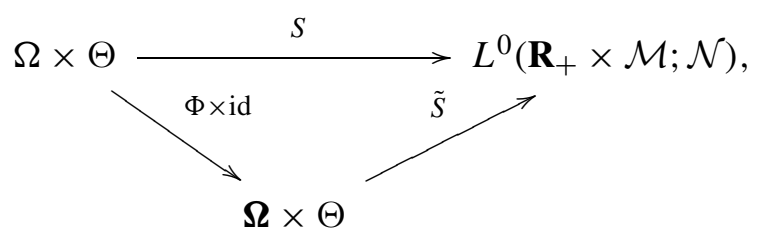

where $\Phi$ is a measurable feature map which "lifts" the path to a rough path and $\tilde{S}$ is a continuous (Lipschitz in some cases) "path-by-path" solution map. The construction of $\tilde{S}$ allows Brownian paths to be treated as a parameter belonging to the set of rough paths, which puts the stochastic and deterministic parameterization schemes on equal footing as far as stability is concerned.

As mentioned above, stochastic parameterization schemes offer a natural mechanism to forecast uncertainty. That is, an ensemble of solutions can be generated. By constructing a 
path-by-path solution map $\tilde{S}$, any element of the enriched space $\boldsymbol{\Omega}$ is an admissible driving path. For example, non-Markovian processes such as fractional Brownian motion have rough path lifts to $\boldsymbol{\Omega}$. Thus, a highly flexible stochastic modeling framework is permissible once the pathwise solution map $\tilde{S}$ is constructed. Recent work on the statistics of 2D fluid turbulence suggests that the subgrid (or fast scales) dynamics of fluids are non-Markovian and nonGaussian [14, 23]. In fact, even piecewise linearly interpolated data from observations could serve as a driving path.

The system of rough partial differential equations we consider in this paper arise from perturbing the advecting vector field in (1.1) by a time-dependent vector field that is rough in time and smooth in space. More precisely, we re-write (1.1) in covariant form, and then perturb the advecting vector field. The perturbation can be understood as a parameterization of the subgrid dynamics of the fluid velocity field. Therefore, the well-posedness and stability results we establish clear the way for the development of a rich and robust modeling framework for fluids.

1.2. Derivation of the equation. In this section we present a heuristic derivation of our main equation and discuss its physical relevance. However, this is not essential for reading and understanding our results in the remainder of the paper and, as such, may be skipped during the first reading.

The Navier-Stokes system (1.1) is the differential form of the momentum balance principle under the additional assumption that the fluid is homogeneous (constant density) and incompressible. The momentum balance in integral-form and in standard coordinates reads

$$
\frac{d}{d t} \int_{\eta_{s, t}(W)} u_{t}^{i} \rho d x=\int_{\eta_{s, t}(W)} v \Delta u_{t}^{i} d x-\int_{\eta_{s, t}(W)} p n^{i} d A=\int_{\eta_{s, t}(W)}\left(v \Delta u_{t}^{i}-\partial_{x^{i}} p_{t}\right) d x,
$$

for all nice regions $W \subset \mathcal{D}$, where we have written the coordinates to emphasize the fact that the momentum balance principle, as stated, is coordinate dependent.

It is a worthwhile endeavor to derive an equation for the momentum balance that is invariant under a change of the coordinate system (see, e.g., for [30] for motivation and [1] or [31] for more details). The language of differential geometry provides the tools to do so, while also providing a natural generalization of the fluid equations to a manifold $M$.

One usually considers the fluid velocity $u$ in (1.1) as a vector field, which we write as $u=u^{j} \frac{\partial}{\partial x_{j}}$. where $(x, U)$ is a local coordinate system of $M$ and $\frac{\partial}{\partial x_{j}}$ is the local basis of the tangent bundle $T M$. Here and for the rest of the paper we use the convention of summation over repeated indices. In the inviscid case $\vartheta=0$, that is, for Euler's equations, the momentum balance principle implies conservation of circulation by Reynold's transport theorem:

$$
\oint_{\eta_{s, t}(C)} u_{t}=\oint_{C} u_{0}
$$

for any $s, t$ and any contour $C$. The reader will notice the ambiguity of the above integralsthe contour is a one-dimensional subset of $M$ and as such one should really understand $u$ as a 1 -form. One can obtain a 1 -form from $u$ on a Riemannian manifold $(M, g)$ by setting $u^{b}:=g_{i j} u^{j} d x^{j}$, where $d x^{j}$ is a local basis of the cotangent bundle $T^{*} M$ and $g_{i j}$ is the metric tensor in local coordinates. To simplify our discussion below, we assume the manifold is flat $g_{i j}=\delta_{i j}$ (globally) and boundaryless (e.g., the Torus). Thus, the contour integrals above can be written as line integrals of the one-form $u^{b}$ :

$$
\oint_{\eta_{s, t}(C)} u_{t}^{b}=\oint_{C} u_{0}^{b}
$$


To obtain a coordinate-free expression for $u^{b}$, we first consider the Navier-Stokes equation in standard coordinates:

$$
\partial_{t} u^{i}+u^{j} \frac{\partial}{\partial x_{j}} u^{i}=-\frac{\partial}{\partial x_{i}} p+\vartheta \Delta u^{i}, \quad i \in\{1, \ldots, d\} .
$$

Adding $u^{j} \frac{\partial}{\partial x_{i}} u^{j}$ to both-sides of the equation, we get

$$
\partial_{t} u^{i}+u^{j} \frac{\partial}{\partial x_{j}} u^{i}+u^{j} \frac{\partial}{\partial x_{i}} u^{j}=-\frac{\partial}{\partial x_{i}} \tilde{p}+\vartheta \Delta u^{i},
$$

where $\tilde{p}=p-\frac{1}{2}|u|^{2}$. The reason for adding this term to both sides is that the last two terms on the left-hand side of the equality can be identified with the Lie derivative of the one-form $u^{b}$ along $u$ :

$$
£_{u_{t}} u_{t}^{\mathrm{b}}=\left.\frac{d}{d \tau}\left(\eta_{t, \tau}\right)^{*} u_{t}^{\mathrm{b}}\right|_{\tau=t}=u_{t}^{j} \frac{\partial}{\partial x_{j}} u_{t}^{i} d x^{i}+u_{t}^{j} \frac{\partial}{\partial x_{i}} u_{t}^{j} d x^{i},
$$

where the latter equality is a direct consequence of Cartan's magic formula. Let $\mathbf{d}$ be the exterior differential operator and $\delta$ the co-differential operator. The operator $\mathbf{d} \delta+\delta \mathbf{d}$ is called the Hodge-Laplacian, and is equal to the (Levi-Civita) connection Laplacian on flat space by the Weitzenböck identity. In particular,

$$
\Delta u=\left((\mathbf{d} \delta+\delta \mathbf{d}) u^{b}\right)^{\sharp} \quad \Leftrightarrow \quad \Delta u^{i}=\left((\mathbf{d} \delta+\delta \mathbf{d}) u^{b}\right)_{i}, \quad i \in\{1, \ldots d\},
$$

where $\sharp$ denotes the inverse of the $b$ operator. Putting it all together, the covariant form of the Navier-Stokes equation is given by

$$
\partial_{t} u^{b}+£_{u} u^{b}=-\mathbf{d} \tilde{p}+\vartheta \delta \mathbf{d} u^{b}, \quad \delta u^{b}=0,
$$

where the divergence-free condition is written in terms of the codifferential. The term $\mathfrak{f}_{u} u^{b}$ is the nonlinear Lie-advection of the one-form $u^{b}$ by the vector-field $u$ whose associated flow generates the integral curves $\eta$. As an application of Reynold's transport theorem, we find

$$
\frac{d}{d t} \oint_{\eta_{s, t}(C)} u_{t}^{b}=\oint_{\eta_{s, t}(C)}\left(\partial_{t}+£_{u_{t}}\right) u_{t}^{\mathrm{b}}=\oint_{\eta_{s, t}(C)}\left(\mathbf{d} \tilde{p}+\vartheta(\mathbf{d}+\delta)^{2} u^{b}\right),
$$

which, upon applying Stokes' theorem, gives a convenient proof of circulation conservation when $\vartheta=0$.

In practice, one must approximate solutions of (1.2), and hence effectively ignore the high modes of the solution. That is, one can only compute solutions of

$$
\partial_{t} u^{L, b}+£_{u^{L}} u^{L, b}=-\mathbf{d} \tilde{p}+\vartheta \delta \mathbf{d} u^{L, b}, \quad \delta u^{L, b}=0,
$$

where $u^{L}$ has only modes up to a certain order. A way of improving approximations on a limited computational budget is to parameterize the high-modes $u^{H}$ of $u$ by a vector field $\tilde{u}^{H}$ and compute

$$
\partial_{t} u^{L, b}+£_{u^{L}+\tilde{u}^{H}} u^{L, b}=-\mathbf{d} \tilde{p}+\vartheta \delta \mathbf{d} u^{L, b}, \quad \delta u^{L, b}=0 .
$$

One possible choice of a parameterization of $u^{H}$ is given by $\tilde{u}^{H}=\sigma_{k} \dot{B}_{t}^{k}$, where $\sigma_{k}: M \rightarrow$ $\mathbf{R}^{d}, k \in\{1, \ldots, K\}$, are sufficiently regular divergence-free vector fields and $B^{k}: \mathbf{R}_{+} \rightarrow \mathbf{R}$ are independent Brownian motions. The hope is that $u$ is approximated by a stochastic ensemble of solutions of $u^{L}$. In fact, such an equation can be derived from the theory of stochastic homogenization combined with a variational principle, and we refer the reader to $[8,9,22]$ for more details about the derivation and for verifiable proof that the parameterization is flexible enough to capture the high-modes of $u$. Motivated by the practical success of this approach, we seek to develop a framework for more flexible parameterizations, where instead 
of Brownian motions $B^{k}$, one considers rough paths $z^{k}$, and to develop pathwise stability of the Brownian case, at least in dimension two.

Motivated by this problem, we perturb the advecting vector field $u$ in $\mathfrak{f}_{u} u^{b}$ in (1.2) by a random vector field of the form $\sigma_{k} \dot{z}^{k}$, where $\sigma_{k}: M \rightarrow \mathbf{R}^{d}, k \in\{1, \ldots, K\}$, are sufficiently regular divergence-free vector fields and $z^{k}: \mathbf{R}_{+} \rightarrow \mathbf{R}$ are driving paths, which shall eventually possess only a limited regularity. That is, we replace $£_{u} u^{b}$ with $£_{u+\sigma_{k}} \dot{z}^{k} u^{b}$ and consider

$$
\partial_{t} u^{b}+£_{u+\sigma_{k}} \dot{z}^{k} u^{\mathrm{b}}=\partial_{t} u^{\mathrm{b}}+£_{u} u^{\mathrm{b}}+£_{\sigma_{k}} u^{\mathrm{b}} \dot{z}_{t}^{k}=-\mathbf{d} \tilde{p}+\vartheta \delta \mathbf{d} u^{\mathrm{b}} .
$$

The vector field $u+\sigma_{k} \dot{z}^{k}$ generates the two-parameter flow $\tilde{\eta}$ on $M$ :

$$
\dot{\tilde{\eta}}_{s, t}(x)=u_{t}\left(\tilde{\eta}_{s, t}(x)\right)+\sigma_{k}\left(\tilde{\eta}_{s, t}\right) \dot{z}_{t}^{k}, \quad \tilde{\eta}_{s, s}(x)=x, \quad s \leq t, x \in M .
$$

We understand this on a formal level, since it is not clear how to construct the flow map $\tilde{\eta}$ due to the low regularity. Applying Reynold's transport theorem, we find

$$
\frac{d}{d t} \oint_{\tilde{\eta}_{s, t}(C)} u_{t}^{b}=\oint_{\eta_{s, t}(C)}\left(\partial_{t}+£_{u+\sigma_{k} \dot{z}^{k}}\right) u_{t}^{\mathrm{b}}=\oint_{\eta_{s, t}(C)}\left(\mathbf{d} \tilde{p}+\vartheta(\mathbf{d}+\delta)^{2} u^{\mathrm{b}}\right),
$$

which yields conservation of circulation in the inviscid case $\vartheta=0$ (see also, [10]).

Writing (1.3) in local coordinates, we obtain

$$
\partial_{t} u^{i}+u^{j} \frac{\partial}{\partial x_{j}} u^{i}+\left[\sigma_{k}^{j} \frac{\partial}{\partial x_{j}} u^{i}+u^{j} \frac{\partial}{\partial x_{i}} \sigma_{k}^{j}\right] \dot{z}_{t}^{k}=-\frac{\partial}{\partial x_{i}} p+\vartheta \Delta u^{i},
$$

where we note that we are again writing $p$ (and not $\tilde{p}$ ) which explains that $u^{j} \frac{\partial}{\partial x_{i}} u^{j}$ does not appear in the equation. This is the main equation we study in this paper. In particular, we introduce a formulation of the equation well suited to make sense of the distributional terms $\dot{z}^{k}$ and to study well-posedness (see (3.1)).

However, for technical reasons related to the noise term $\dot{z}^{k}$, the nonlocal nature of the pressure term (which translates to the divergence-free condition) makes it difficult to obtain a priori estimates directly from this formulation. We elaborate on this issue a bit more in Section 1.3.

One way to circumvent dealing with the pressure is to consider the 2 -form $\tilde{\xi}=\mathbf{d} u^{\mathrm{b}}$, called the vorticity. Taking the exterior derivative in (1.3) and using that $\mathbf{d}$ commutes with the Lie derivative, we get

$$
\partial_{t} \tilde{\xi}+£_{u} \tilde{\xi}+£_{\sigma_{k}} \tilde{\xi} \dot{z}_{t}^{k}=\vartheta \mathbf{d} \delta \tilde{\xi}
$$

Let us consider the Hodge star of the vorticity, which we denote by $\xi$, and is equal to the scalar $* \tilde{\xi}$ in dimension two and the vector field $(* \xi)^{\#}$ in dimension three, where $*$ is the Hodge-star operator, which maps 2-forms to $d-2$-forms. It follows that (see, e.g., pages 451 and 566 in [31] or Appendix A.6. of [4] which proves $\left[\sharp \star^{d-2}, \mathfrak{£}_{v}\right]=0$ and recall that we have assumed flatness)

$$
\partial_{t} \xi+£_{u} \xi+£_{\sigma_{k}} \xi \dot{z}_{t}^{k}=\vartheta \Delta \xi
$$

where $£_{\sigma_{k}} \xi=\sigma_{k}(\xi)$ in dimension two since $\xi$ is a scalar and $£_{\sigma_{k}} \xi=\left[\sigma_{k}, \xi\right]$ in dimension three since $\xi$ is a vector field, and we have slightly abused notation in writing the Laplacian on the right-hand-side. In standard coordinates, $\xi$ solves a scalar transport equation in dimension two:

$$
\partial_{t} \xi+u^{j} \frac{\partial}{\partial x_{j}} \xi+\sigma_{k}^{j} \frac{\partial}{\partial x_{j}} \xi \dot{z}_{t}^{k}=\vartheta \Delta \xi
$$


and $\xi$ solves a perturbed version of the usual vorticity equation in dimension three:

$$
\begin{aligned}
\left(\partial_{t} \xi+\left[u+\sigma_{k} \cdot z^{k}, \xi\right]\right)^{i} & =\partial_{t} \xi^{i}+u^{j} \frac{\partial}{\partial x_{j}} \xi^{i}-\xi^{j} \frac{\partial}{\partial x_{j}} u^{i}+\left[\sigma_{k}^{j} \frac{\partial}{\partial x_{j}} \xi^{i}-\xi^{j} \frac{\partial}{\partial x_{j}} \sigma_{k}^{i}\right] \dot{z}_{t}^{k} \\
& =\vartheta \Delta \xi^{i}, \quad i \in\{1,2,3\} .
\end{aligned}
$$

The reader will notice that the difference between $d=2$ and $d=3$ is the presence of the formidable vorticity stretching terms $\xi^{j} \frac{\partial}{\partial x_{j}} u^{i}$ and $\xi^{j} \frac{\partial}{\partial x_{j}} \sigma_{k}^{i}$ in $d=3$, whose presence causes difficulty from the analytic point of view, but interesting dynamics from the modeling point of view. For convenience (and with a slight abuse of notation) we abbreviate the two equations for $\xi$ as

$$
\partial_{t} \xi+(u \cdot \nabla) \xi-\mathbf{1}_{d=3}(\xi \cdot \nabla) u+\left[\left(\sigma_{k} \cdot \nabla\right) \xi-\mathbf{1}_{d=3}(\xi \cdot \nabla) \sigma_{k}\right] \dot{z}_{t}^{k}=\vartheta \Delta \xi
$$

and we note that there is no nonlocality, meaning no pressure term which would influence the noise. In dimension two, by formally testing against $\xi$ and using the fact that the $\sigma_{k}$ are divergence-free, we find

$$
\left|\xi_{t}\right|_{L^{2}}^{2}+2 \vartheta \int_{0}^{t}\left|\nabla \xi_{r}\right|_{L^{2}}^{2} d r=\left|\xi_{0}\right|_{L^{2}}^{2}
$$

which implies that enstrophy is balanced in dimension two.

Equation (1.5) is, of course, still nonlinear due to the presence of $\mathfrak{f}_{u}$, so one needs to write $u$ in terms of $\xi$. This operation, which is the Biot-Savart operator and acts as an inverse of d, can only be done up to additive constants since $\mathbf{d}$ is a derivative (that is, in the Hodge decomposition, there is a Harmonic part). More precisely, on the Torus, one can see that the missing constant is the spatial average of $u$, which by formally integrating (1.4) in space, should satisfy

$$
\partial_{t} \int_{\mathbf{T}^{d}} u_{t}^{i}(x) d x+\int_{\mathbf{T}^{d}} u_{t}^{j}(x) \frac{\partial}{\partial x_{i}} \sigma_{k}^{j}(x) d x \dot{z}_{t}^{k}=0
$$

when we assume $u$ and $\sigma_{k}$ are divergence-free. Notice that there is no geometric ambiguity in the above spatial integrals since we are considering the components of $u$.

Throughout our analysis, it is therefore necessary to preserve the information in (1.6) as it allows us to recover the full velocity. In other words, we solve (1.5) and (1.6) as a system of equations, which is better suited for deriving a priori estimates of (1.5), which from now on will be referred to as enstrophy estimates. In addition, the system (1.5), (1.6) is shown to be equivalent to (1.4) under the condition $\nabla \cdot u=0$. We note that there this issue does not appear in the classical Navier-Stokes equations, that is, in the case $z^{k}=0$. Indeed, equation (1.6) shows that the Navier-Stokes system conserves the spatial average so that one may without loss of generality assume that $\int_{M} u_{0}^{i}(x) d x=0$.

1.3. Related literature and main contributions. The stochastic Navier-Stokes equation has been well studied using Brownian motion as the driving noise. With no ambition at an exhaustive list of references, let us mention [5, 6, 15, 28, 29]. Moreover, a similar multiplicative noise as in the present paper has been studied in [10] and [7]. In the pathwise setting, using regularity structures, the Navier-Stokes system with space-time white noise has been studied in [33].

A problem similar to (1.4), namely,

$$
\begin{aligned}
\partial_{t} u+(u \cdot \nabla) u+\left(\sigma_{k} \cdot \nabla\right) u \dot{z}_{t}^{k} & =-\nabla p+\vartheta \Delta u, \\
\nabla \cdot u=0,\left.\quad u\right|_{t=0} & =u_{0},
\end{aligned}
$$


has been studied by the same authors in [21]. On the surface, the main difference between (1.4) and (1.7) is that the noise in (1.7) is energy conservative for the velocity. However, based on the discussion in Section 1.2, we see that, in general, the perturbation does not conserve circulation in the inviscid case nor enstrophy balance in dimension two. In fact, (1.7) is usually obtain by treating the solution of Navier-Stokes as a collection of scalar equations, thus ignoring the geometry of the problem (i.e., the Lie derivative). Furthermore, there are deep, highly technical and structural reasons why energy conservation for the velocity does not yield satisfactory well-posedness results.

More precisely, as it will become clear in the derivation below in Section 3, applying the Helmholtz projection to the equation entangles a nonlocality into the rough integral term. The only available method ${ }^{1}$ to obtain uniqueness of weak solutions for rough PDEs in the variational setting is the method introduced in [2], based on commutator estimates à la DiPerna and Lions [13]. However, this approach seems to fail under the presence of the Helmholtz projection. Consequently, uniqueness in [21] could only be proved under very restrictive assumptions on the vector fields $\sigma_{k}$, namely the ones for which the rough term commutes with the Helmholtz projection, effectively restricting to constant vector fields.

Leaving this aside, there is also a structural problem with the equation containing the projection, even if one could use the techniques of [13]. Indeed, since the Helmholtz projection is not continuous on $L^{\infty}$, the equation for $u u^{T}$, which is needed for the energy estimates, contains noise that cannot be made sense of in an appropriate Banach space as for instance $\left(L^{\infty}\right)^{*}$ that is dictated by the deterministic part of the equation. To summarize, it is the unfavorable interplay between the energy conservative noise and the deterministic part of the equation reflected through the Helmholtz projection, which makes the problem not easily accessible for a direct pathwise analysis.

In the present paper, we take a different path and develop a model in which the noise conserves circulation in the inviscid limit (which we do not address in this paper) and enstrophy balance in dimension two. Note that the enstrophy corresponding to the $L^{2}$-norm of the vorticity is balanced in two space dimensions and conserved in case $\vartheta=0$ as in the deterministic unforced setting. Enstrophy, however, is not balanced in three dimensions, which leads to a significantly more involved analysis and only local in time solutions. Moreover, since the vorticity formulation eliminates the pressure, the nonlocality of the equation for the vorticity does not influence the noise-term. On the other hand, as discussed above, particular care has to be taken in order to fully recover the velocity from the vorticity formulation on the Torus. This subtlety seems to have been missed in the available literature.

Additionally, we establish pathwise continuity properties which easily enables the study of Wong-Zakai results for the case of Brownian motion with Stratonovich integration in dimension two. Furthermore, the generation of a continuous random dynamical system from the unique solution follows for a large class of driving stochastic processes. This set up could also be used for studying large deviations and support theorems.

Another contribution of this paper is that it develops the theory of unbounded rough drivers as introduced in [2] and further developed in [12] - the unbounded rough driver theory is a method to study PDEs perturbed by an unbounded operator-valued noise term. Still, an abstract variational method in the spirit of [24] for these equations is not available. That we had to tailor the method of unbounded rough drivers to the Navier-Stokes equation in a nontrivial way is an indication why a general theory is not yet available. On the other hand, exactly this fact of being able to tune the method demonstrates its flexibility and suggests

\footnotetext{
${ }^{1}$ An alternative method has been introduced in [20], but it is not clear whether it is applicable to systems or how to encode the divergence-free condition.
} 
that this mentality could be used for studying other equations, and possibly build towards a general theory.

The paper is organized as follows. Section 2 is devoted to notation and definitions. The precise formulation of the problem, derivation of the vorticity formulation and the main results are described in Section 3. Section 4 contains basic a priori estimates. Enstrophy balance, uniqueness as well as the rough path stability and Wong-Zakai result in two space dimensions is presented in Section 5, whereas Section 6 contains the proof of existence. Certain auxiliary results are collected in the Appendix.

2. Preliminaries. In this section, we introduce the notation and collect the basic definitions needed in the sequel.

2.1. Sobolev spaces and vector calculus. We begin by fixing the notation that we use throughout the paper.

For a given $d \in\{2,3\}$, let $\mathbf{T}^{d}=\mathbf{R}^{d} /(2 \pi \mathbf{Z})^{d}$ be the $d$-dimensional flat torus and denote by $d x$ the unnormalized Lebesgue measure on $\mathbf{T}^{d}$. As usual, we blur the distinction between periodic functions defined on the whole space and functions defined on the torus $\mathbf{T}^{d}$. We let $\partial_{i}$ denote the partial derivative $\frac{\partial}{\partial x_{i}}$ in the $i$ th standard direction.

For a given $m \in \mathbf{Z}$ and $d^{\prime} \in \mathbf{N}$, we define the Sobolev spaces $W^{m, 2}\left(\mathbf{T}^{d} ; \mathbf{R}^{d^{\prime}}\right)=(I-$ $\Delta)^{-\frac{m}{2}} L^{2}\left(\mathbf{T}^{d} ; \mathbf{R}^{d^{\prime}}\right)$ and denote by $|\cdot|_{m}$ the corresponding norm. We let $W^{m, 2}=W^{m, 2}\left(\mathbf{T}^{d} ; \mathbf{R}\right)$ and $\mathbf{W}^{m, 2}=W^{m, 2}\left(\mathbf{T}^{d} ; \mathbf{R}^{d}\right)$. For a given $m \in \mathbf{N}$ and $d^{\prime} \in \mathbf{N}$, we denote by $W^{m, \infty}\left(\mathbf{T}^{d} ; \mathbf{R}^{d^{\prime}}\right)$ the Sobolev space of functions whose weak-derivatives up to and including order $m$ are in $L^{\infty}\left(\mathbf{T}^{d} ; \mathbf{R}^{d^{\prime}}\right)$. For $m<0$, we define $W^{m, \infty}\left(\mathbf{T}^{d} ; \mathbf{R}^{d^{\prime}}\right)=\left(W^{-m, \infty}\left(\mathbf{T}^{d} ; \mathbf{R}^{d^{\prime}}\right)\right)^{*}$. We denote the corresponding norms of $W^{m, \infty}\left(\mathbf{T}^{d} ; \mathbf{R}^{d^{\prime}}\right)$ by $|\cdot|_{m, \infty}$. Moreover, for $m \geq 0$, we denote by $\mathbf{W}_{\text {div }}^{m, \infty}$ the functions in $W^{m, \infty}\left(\mathbf{T}^{d} ; \mathbf{R}^{d}\right)$ that are weakly-divergence free.

The Leray projection onto divergence-free vector fields is denoted by $P$ and is defined by $P u:=\mathrm{Id}-\nabla \Delta^{-1}(\nabla \cdot u)$. We let $Q=I-P$ be the orthogonal projection on to gradient vector fields. We set $\mathbf{H}^{m}=P \mathbf{W}^{m, 2}$ and $\mathbf{H}_{\perp}^{m}=Q \mathbf{W}^{m, 2}$ and recall that for all $m \in \mathbf{Z}$ (see Lemma 3.7 in [27]), $\mathbf{W}^{m, 2}=\mathbf{H}^{m} \oplus \mathbf{H}_{\perp}^{m}$, where

$$
\mathbf{H}^{m}=\left\{f \in \mathbf{W}^{m, 2}: \nabla \cdot f=0\right\} \quad \text { and } \quad \mathbf{H}_{\perp}^{m}=\left\{g \in \mathbf{W}^{m, 2}:(f, g)=0, \forall f \in \mathbf{H}^{-m}\right\} .
$$

We denote by $\dot{\mathbf{H}}^{m}$ the subspace of functions in $\mathbf{H}^{m}$ that are mean-free $\bar{f}:=\int_{\mathbf{T}^{d}} f(x) d x=$ 0 . Similarly, we define $\dot{\mathbf{W}}^{m, 2}$ and $\dot{W}^{m, 2}$. However, we often abuse notation and use $\dot{\mathbf{H}}^{m, 2}$ for scalar functions when it is clear from the context (see Remark 2.1).

Let $\sigma: \mathbf{T}^{d} \rightarrow \mathbf{R}^{d}$ and $c: \mathbf{T}^{d} \rightarrow \mathbf{R}^{d \times d}$ be twice differentiable and assume that the derivatives up to order two are bounded uniformly. Define the operators

$$
\mathcal{M} \phi=\sigma \cdot \nabla \phi+c \phi=\sum_{i=1}^{d} \sigma^{i} \partial_{i} \phi+c \phi, \quad \mathcal{M}^{2} \phi=(\sigma \cdot \nabla+c)^{2} \phi .
$$

It follows that there is a constant $N=N\left(|\sigma|_{2, \infty},|c|_{2, \infty}\right)$ such that

$$
\begin{aligned}
&|\mathcal{M}|_{\mathcal{L}\left(\mathbf{W}^{m+1,2}, \mathbf{W}^{m, 2}\right)} \leq N, m=0,1,2, \\
&\left|\mathcal{M}^{2}\right|_{\mathcal{L}\left(\mathbf{W}^{m+2,2}, \mathbf{W}^{m, 2}\right)} \leq N, \quad m=-1,0,1,
\end{aligned}
$$

where $\mathcal{L}\left(V, V^{\prime}\right)$ denotes the Banach space of bounded linear operators between two arbitrary Banach spaces $V$ and $V^{\prime}$. We note that there is also a constant $N=N\left(|\sigma|_{2, \infty},|c|_{2, \infty}\right)$ such that

$$
|\mathcal{M}|_{\mathcal{L}\left(\mathbf{W}^{m+1, \infty}, \mathbf{W}^{m, \infty}\right)} \leq N, \quad m=0,1,2, \quad\left|\mathcal{M}^{2}\right|_{\mathcal{L}\left(\mathbf{W}^{m+2,2}, \mathbf{W}^{m, \infty}\right)} \leq N, \quad m=0,1
$$


Because $P \in \mathcal{L}\left(\mathbf{W}^{m, 2}, \mathbf{H}^{m}\right)$ and $Q \in \mathcal{L}\left(\mathbf{W}^{m, 2}, \mathbf{H}_{\perp}^{m}\right)$ for all $m \in \mathbf{Z}$, both of which have operator norm bounded by 1 , we have

$$
\begin{array}{ll}
\left|P \mathcal{M}^{1}\right|_{\mathcal{L}\left(\mathbf{H}^{m+1}, \mathbf{H}^{m}\right)} \leq N, & m=0,1,2, \\
\left|P \mathcal{M}^{2}\right|_{\mathcal{L}\left(\mathbf{H}^{m+2}, \mathbf{H}^{m}\right)} \leq N, \quad m=-1,0,1, \\
\left|Q \mathcal{M}^{1}\right|_{\mathcal{L}\left(\mathbf{H}_{\perp}^{m+1}, \mathbf{H}_{\perp}^{m}\right)} \leq N, \quad m=0,1,2, \\
\left|Q \mathcal{M}^{2}\right|_{\mathcal{L}\left(\mathbf{H}_{\perp}^{m+2}, \mathbf{H}_{\perp}^{m}\right)} \leq N, \quad m=-1,0,1 .
\end{array}
$$

For a given $u: \mathbf{T}^{d} \rightarrow \mathbf{R}^{d}$ with $d \in\{2,3\}$, the exterior derivative coincides with the curl operator and can be written in standard coordinates as

$$
\begin{aligned}
& \operatorname{curl} u=\nabla \times u=\left(\partial_{2} u^{3}-\partial_{3} u^{2}, \partial_{3} u^{1}-\partial_{1} u^{3}, \partial_{1} u^{2}-\partial_{2} u^{1}\right) \quad \text { if } d=3, \\
& \operatorname{curl} u=\nabla \times u=\partial_{1} u^{2}-\partial_{2} u^{1} \quad \text { if } d=2 .
\end{aligned}
$$

We also note that $\nabla \times P=\nabla \times$.

REMARK 2.1. We could equivalently define $\nabla \times$ only for $d=3$ and then embed twodimensional vector fields into $\mathbf{R}^{3}$ by $\left(u^{1}, u^{2}\right) \mapsto\left(u^{1}, u^{2}, 0\right)$. This also justifies why we abuse notation and write $\nabla \times u \in \dot{\mathbf{H}}^{m}$ even in dimension two because $\nabla \times u$ is divergence free when a two-dimensional vector field is embedded as above.

We define the Biot-Savart operator (i.e., inverse of the curl) of a given mean-free function $f: \mathbf{T}^{2} \rightarrow \mathbf{R}$ by

$$
\mathcal{K} f=\nabla^{\perp}(-\Delta)^{-1} f
$$

where $\nabla^{\perp} f:=\partial_{2} f \mathbf{i}-\partial_{1} f \mathbf{j}$. We define the Biot-Savart operator (i.e., inverse of the curl) of a given mean-free function $f: \mathbf{T}^{3} \rightarrow \mathbf{R}$ by

$$
\mathcal{K} f=\nabla \times(-\Delta)^{-1} f .
$$

It follows that for a given mean-free $f$ :

$$
\begin{aligned}
& \nabla \times(-\Delta)^{-1} \mathcal{K} f=(-\Delta)^{-1} f \quad \text { if } d=2, \\
& \mathcal{K}^{2} f=(-\Delta)^{-1} f \quad \text { if } \nabla \cdot f=0 \text { and } d=3 .
\end{aligned}
$$

For all $n \in \mathbf{N}$, we have

$$
\mathcal{K} \in \mathcal{L}\left(\dot{\mathbf{H}}^{n-1,2}, \dot{\mathbf{H}}^{n}\right), \quad \operatorname{curl} \in \mathcal{L}\left(\mathbf{W}^{n, 2}, \dot{\mathbf{H}}^{n-1}\right),
$$

and

$$
|\nabla \mathcal{K} f|_{n}=|f|_{n} .
$$

Moreover, for all $n \in \mathbf{N}$, curl $\circ \mathcal{K} \in \mathcal{L}\left(\dot{\mathbf{H}}^{n-1,2}, \dot{\mathbf{H}}^{n-1}\right)$ is the identity operator if $d=2$ and restricts to the identity operator on $\dot{\mathbf{H}}^{n-1}$ if $d=3$, and $\mathcal{K} \circ$ curl $\in \mathcal{L}\left(\mathbf{W}^{n, 2}, \dot{\mathbf{H}}^{n}\right)$ restricts to the identity on $\dot{\mathbf{H}}^{n}$ if $d \in\{2,3\}$.

In order to analyze the nonlinear term in (1.4), we employ the classical notation and bounds. Owing to Lemma 2.1 in [32], the trilinear form

$$
b(u, v, w)=\int_{\mathbf{T}^{d}}((u \cdot \nabla) v) \cdot w d x=\sum_{i, j=1}^{d} \int_{\mathbf{T}^{d}} u^{i} \partial_{i} v^{j} w^{j} d x
$$


satisfies the continuity property

$$
|b(u, v, w)| \lesssim m_{1}, m_{2}, m_{3}, d|u|_{m_{1}}|v|_{m_{2}+1}|w|_{m_{3}}, \quad m_{1}+m_{2}+m_{3}>\frac{d}{2}, m_{1}, m_{2}, m_{3} \geq 0 .
$$

Moreover, for all $u \in \mathbf{H}^{m_{1}}$ and $(v, w) \in \mathbf{W}^{m_{2}+1,2} \times \mathbf{W}^{m_{3}, 2}$ such that $m_{1}, m_{2}, m_{3}$ satisfy (2.4), we have

$$
b(u, v, w)=-b(u, w, v) \quad \text { and } \quad b(u, v, v)=0 .
$$

For $m_{1}, m_{2}$, and $m_{3}$ that satisfy (2.4), we obtain a bilinear mapping $B: \mathbf{W}^{m_{1}, 2} \times \mathbf{W}^{m_{2}+1,2} \rightarrow$ $\mathbf{W}^{-m_{3}, 2}$ defined by $(B(u, v), w)=b(u, v, w)$. We define $B_{P}=P B$ and $B_{Q}=Q B$ and giving the continuous bilinear mappings

$$
B_{P}: \mathbf{W}^{m_{1}, 2} \times \mathbf{W}^{m_{2}+1,2} \rightarrow \mathbf{H}^{-m_{3}}, \quad B_{Q}: \mathbf{W}^{m_{1}, 2} \times \mathbf{W}^{m_{2}+1,2} \rightarrow \mathbf{H}_{\perp}^{-m_{3}} .
$$

We set $B(u)=B(u, u)$ and similarly for $B_{P}$ and $B_{Q}$.

2.2. Rough paths. For an interval $I$, we use the notation $\Delta_{I}:=\left\{(s, t) \in I^{2}: s \leq t\right\}$ and $\Delta_{I}^{(2)}:=\left\{(s, \theta, t) \in I^{3}: s \leq \theta \leq t\right\}$. For simplicity, we let $\Delta_{T}:=\Delta_{[0, T]}$ and $\Delta_{T}^{(2)}=\Delta_{[0, T]}^{(2)}$ for $T>0$. Let $E$ be a Banach space with norm $|\cdot|_{E}$. A function $g: \Delta_{I} \rightarrow E$ is said to have finite $p$-variation for some $p>0$ on $I$ if

$$
|g|_{p-\operatorname{var} ; I ; E}:=\sup _{\left(t_{i}\right) \in \mathcal{P}(I)}\left(\sum_{i}\left|g_{t_{i} t_{i+1}}\right|_{E}^{p}\right)^{\frac{1}{p}}<\infty,
$$

where $\mathcal{P}(I)$ is the set of all partitions of $I$. We denote by $C_{2}^{p \text {-var }}(I ; E)$ the set of all continuous functions with finite $p$-variation on $\Delta_{I}$ equipped with the seminorm $|\cdot|_{p \text {-var; } I ; E}$ and by $C^{p \text {-var }}(I ; E)$ the set of all paths $z: I \rightarrow E$ such that $\delta z \in C_{2}^{p \text {-var }}(I ; E)$, where $\delta z_{s t}:=z_{t}-z_{s}$. In this section, we drop the dependence of norms on the space $E$ when convenient.

A continuous mapping $\omega: \Delta_{I} \rightarrow[0, \infty)$ is called a control on $I$ provided $\omega(s, s)=0$ and it is superadditive, namely

$$
\omega(s, \theta)+\omega(\theta, t) \leq \omega(s, t), \quad s \leq \theta \leq t .
$$

If for a given $p>0, g \in C_{2}^{p \text {-var }}(I ; E)$, then it can be shown that the 2-index map $\omega_{g}: \Delta_{I} \rightarrow$ $[0, \infty)$ defined by

$$
\omega_{g}(s, t)=|g|_{p-\operatorname{var} ;[s, t]}^{p}
$$

is a control (see, e.g., Proposition 5.8 in [17]). Moreover, it is straightforward to check that one could equivalently define the semi-norm on $C_{2}^{p \text {-var }}(I ; E)$ by

$$
\begin{aligned}
& |g|_{p \text {-var; }[s, t]} \\
& \quad=\inf \left\{\omega(s, t)^{\frac{1}{p}}: \omega \text { is a control s.t. }\left|g_{u v}\right| \leq \omega(u, v)^{\frac{1}{p}} \text { for all }(u, v) \in \Delta_{[s, t]}\right\} .
\end{aligned}
$$

We shall need the following local version of the $p$-variation spaces.

DEFINITION 2.2. Given an interval $I=[a, b]$, a control $\varpi$ and real number $L>0$, we denote by $C_{2, \varpi, L}^{p \text {-var }}(I ; E)$ the space of continuous two-index maps $g: \Delta_{I} \rightarrow E$ for which there exists a control $\omega$ such that for every $(s, t) \in \Delta_{I}$ with $\varpi(s, t) \leq L$, it holds that $\left|g_{s t}\right|_{E} \leq$ $\omega(s, t)^{\frac{1}{p}}$. We define a semi-norm on this space by

$|g|_{p-\operatorname{var}, \varpi, L ; I}$

$$
=\inf \left\{\omega(a, b)^{\frac{1}{p}}: \omega \text { is a control s.t. }\left|g_{s t}\right| \leq \omega(s, t)^{\frac{1}{p}}, \forall(s, t) \in \Delta_{I} \text { with } \varpi(s, t) \leq L\right\} .
$$


It follows that for $\varpi_{1} \leq \varpi_{2}$ and $L_{2} \leq L_{1}$,

$$
C_{2, \varpi_{1}, L_{1}}^{p \text {-var }}(I ; E) \subset C_{2, \varpi_{2}, L_{2}}^{p-\text { var }}(I ; E) .
$$

Next, we present the definition of a rough path and the reader is referred to [16, 17, 25] for a thorough exposition of the theory of rough paths. For a two-index map $g: \Delta_{I} \rightarrow \mathbf{R}$, we define the second order increment operator

$$
\delta g_{s \theta t}=g_{s t}-g_{\theta t}-g_{s \theta}, \quad(s, \theta, t) \in \Delta_{I}^{(2)} .
$$

Definition 2.3. Let $K \in \mathbf{N}$ and $p \in[2,3)$. A continuous $p$-rough path is a pair

$$
\mathbf{Z}=(Z, \mathbb{Z}) \in C_{2}^{p \text {-var }}\left([0, T] ; \mathbf{R}^{K}\right) \times C_{2}^{\frac{p}{2}-\operatorname{var}}\left([0, T] ; \mathbf{R}^{K \times K}\right)
$$

that satisfies the Chen's relation

$$
\delta Z_{s \theta t}=0, \quad \delta \mathbb{Z}_{s \theta t}=Z_{s \theta} \otimes Z_{\theta t} \quad \forall(s, \theta, t) \in \Delta_{I}^{(2)} .
$$

We will denote by $\omega_{Z}$ the smallest control dominating both $\left|Z_{s t}\right|^{p}$ and $\left|\mathbb{Z}_{s t}\right|^{\frac{p}{2}}$. Given a smooth path $z$, there is a canonical lift to a rough path $(Z, \mathbb{Z})$, where

$$
Z_{s t}=\delta z_{s t}, \quad \mathbb{Z}_{s t}:=\int_{s}^{t} \delta z_{s r} \otimes \dot{z}_{r} d r=\int_{s}^{t} \delta z_{s r} \otimes d z_{r},
$$

for which Chen's relation is readily checked. Above we have used the increment notation $\delta z_{s t}:=z_{t}-z_{s}$ for a one-index map $z$. A continuous $p$-rough path $\mathbf{Z}=(Z, \mathbb{Z})$ is said to be geometric if it can be obtained as the limit in the product topology $C_{2}^{p \text {-var }}\left([0, T] ; \mathbf{R}^{K}\right) \times$ $C_{2}^{\frac{p}{2}-\operatorname{var}}\left([0, T] ; \mathbf{R}^{K \times K}\right)$ of a sequence of rough paths $\left\{\left(Z^{n}, \mathbb{Z}^{n}\right)\right\}_{n=1}^{\infty}$ that are canonical lifts of some smooth paths $z^{n}:[0, T] \rightarrow \mathbf{R}^{K}$. We denote by $\mathcal{C}_{g}^{p \text {-var }}\left([0, T] ; \mathbf{R}^{K}\right)$ the set of geometric $p$-rough paths and endow it with the product topology.

2.3. Unbounded rough drivers. In [11], A.M. Davie made the groundbreaking observation that rough differential equations can be interpreted as an equation in Taylor expansions. The notion of solution is obtained by iterating a rough differential equation into itself and using Taylor's formula to re-expand nonlinearities in terms of the equation itself. The final expression is an increment equation that allows for detailed analysis of the solution in terms of the oscillations of the temporal noise.

Extending this to the framework of PDEs with unbounded perturbations, we are led to iterating the vector fields acting on the solution. In this setting, the oscillations in time are coupled with spatial derivatives. Thus, one needs appropriate function spaces in order to capture the behavior of the involved quantities with respect to the spatial variable.

Consider a quadruple $\left(E^{n},|\cdot|_{n}\right)_{n=0}^{3}$ of Banach spaces such that $E^{n+k}$ is continuously embedded into $E^{n}$ for $k, n \in\{0,1,2,3\}$ such that $n+k \leq 3$. We denote by $E^{-n}$ the topological dual of $E^{n}$, and note that, in general, $E^{-0} \neq E^{0}$. When the norm is clear from the context, we call $\left(E^{n}\right)_{n}$ a scale of spaces, and it is understood that $n \in\{-3,-2,-1,0,1,2,3\}$.

Definition 2.4. Let $p \in[2,3)$ and $T>0$ be given. A continuous unbounded $p$-rough driver with respect to the scale $\left(E^{n}\right)_{n}$, is a pair $\mathbf{A}=\left(A^{1}, A^{2}\right)$ of 2-index maps such that there exists a continuous control $\omega_{A}$ on $[0, T]$ such that for every $(s, t) \in \Delta_{T}$,

$$
\begin{aligned}
& \left|A_{s t}^{1}\right|_{\mathcal{L}\left(E^{-n}, E^{-(n+1)}\right)}^{p} \leq \omega_{A}(s, t) \quad \text { for } n \in\{0,2\}, \\
& \left|A_{s t}^{2}\right|_{\mathcal{L}\left(E^{-n}, E^{-(n+2)}\right)}^{\frac{p}{2}} \leq \omega_{A}(s, t) \text { for } n \in\{0,1\},
\end{aligned}
$$

and Chen's relation holds true,

$$
\delta A_{s \theta t}^{1}=0, \quad \delta A_{s \theta t}^{2}=A_{\theta t}^{1} A_{s \theta}^{1} \quad \forall(s, \theta, t) \in \Delta_{T}^{(2)} .
$$


We shall need a tool that allows us to compare the regularity of the different spaces in the scale $\left(E^{n}\right)_{n}$.

DEFINITION 2.5. A family of smoothing operators $\left(J^{\eta}\right)_{\eta \in(0,1]}$ acting on $\left(E^{n}\right)_{n}$ is a family of operators such that,

$$
\left|\left(I-J^{\eta}\right) f\right|_{n} \lesssim \eta^{k}|f|_{n+k} \quad \text { and } \quad\left|J^{\eta} f\right|_{n+k} \lesssim \eta^{-k}|f|_{n},
$$

for $0 \leq k \leq 2$ and $-2 \leq n \leq 2$ such that $-3 \leq n+k \leq 3$.

In the scale $\left(\mathbf{H}^{n}\right)_{n}$, a family of self-adjoint smoothing operators can be constructed using the frequency cut-off (see, e.g., [21]). In fact, in this case (2.9) is valid for all $k, n \in \mathbb{Z}$. In the case of the $L^{\infty}$-scale $\left(\mathbf{W}^{n, \infty}\right)_{n}$, one may employ convolution with a nonnegative smoothing kernel to obtain (2.9) for $k \in\{0,1,2\}$.

We include here the main a priori estimate from [12], Theorem 2.10. See, however, Lemma 4.1 for a related result.

\section{THEOREM 2.6. Assume}

- $\left(E^{n}\right)_{n}$ is a scale of spaces for which there exists a family of smoothing operators;

- $\mathbf{A}=\left(A^{1}, A^{2}\right)$ is an unbounded p-rough driver on $\left(E^{n}\right)_{n}$ for $p \in[2,3)$;

- $\mu: I \rightarrow E^{-1}$ is of bounded 1-variation (i.e., $\left|\delta \mu_{s t}\right|_{-1} \leq \omega_{\mu}(s, t)$ for some control $\left.\omega_{\mu}\right)$;

- $g: I \rightarrow E^{-0}$ is a bounded path such that

$$
d g_{t}=d \mu_{t}+\mathbf{A}(d t) g_{t}
$$

in the sense that

$$
g_{s t}^{\natural}:=\delta g_{s t}-\delta \mu_{s t}-A_{s t}^{1} g_{s}-A_{s t}^{2} g_{s}
$$

belongs to $C_{2, \omega_{A}, L}^{\frac{p}{3}-v a r}\left(I ; E^{-3}\right)$ for some $L>0$.

Then there is a positive constant $\tilde{L}=\tilde{L}(p)<L$ such that for all $(s, t) \in \Delta_{I}$ with $\omega_{A}(s, t) \leq$ $\tilde{L}$, we have

$$
\left|g_{s t}^{\natural}\right|_{-3} \lesssim p|g|_{L^{\infty}\left(I ; E^{-0}\right)} \omega_{A}(s, t)^{\frac{3}{p}}+\omega_{\mu}(s, t) \omega_{A}(s, t)^{\frac{1}{p}}
$$

3. Formulation of the problem and the main results. As the first step of our analysis, we derive a rough path formulation of (1.4) and (1.5), which will be satisfied by solutions constructed in our main existence result below, Theorem 3.7. For notational convenience, we change the sign of the vector fields $\sigma_{k}$, so that (1.4) becomes

$$
\begin{aligned}
\partial_{t} u_{t}+\left(u_{t} \cdot \nabla\right) u_{t}+\nabla p_{t} & =\vartheta \Delta u_{t}+\left[\left(\sigma_{k} \cdot \nabla\right) u_{t}+\left(\nabla \sigma_{k}\right) u_{t}\right] \dot{z}_{t}^{k}, \\
\nabla \cdot u_{t} & =0,\left.\quad u_{t}\right|_{t=0}=u_{0},
\end{aligned}
$$

for a given initial condition $u_{0}$ and divergence-free vector fields $\sigma_{k}, k \in\{1, \ldots, K\}$. The unknowns in (3.1) the velocity field $u:[0, T] \times \mathbf{T}^{d} \rightarrow \mathbf{R}^{d}$ and the pressure $p:[0, T] \times$ $\mathbf{T}^{d} \rightarrow \mathbf{R}$. We note that the $i$ th component of $\left(\nabla \sigma_{k}\right) u_{t}$ is given by $\partial_{i} \sigma_{k} \cdot u_{t}=\partial_{i} \sigma_{k}^{j} u_{t}^{j}$ so that componentwise $(3.1)$ reads

$$
\partial_{t} u_{t}^{i}+u_{t}^{j} \partial_{j} u_{t}^{i}+\partial_{i} p_{t}=\vartheta \Delta u_{t}^{i}+\left[\sigma_{k}^{j} \partial_{j} u_{t}+\partial_{i} \sigma_{k}^{j} u_{t}^{j}\right] \dot{z}_{t}^{k}, \quad i \in\{1,2, \ldots d\},
$$

where there is an implicit summation over the repeated indices $j, k$. We always consider $d \in\{2,3\}$. 
We study the Navier-Stokes equation in the variational framework by decoupling the velocity field and the pressure into two equations using the Leray projection $P$ defined in Section 2.1. Applying the solenoidal $P: \mathbf{W}^{m, 2} \rightarrow \mathbf{H}^{m}$ and gradient projection $Q: \mathbf{W}^{m, 2} \rightarrow \mathbf{H}_{\perp}^{m}$ separately to (3.1) yields

$$
\begin{aligned}
& \partial_{t} u_{t}+P\left[\left(u_{t} \cdot \nabla\right) u_{t}\right]=\vartheta \Delta u_{t}+P\left[\left(\sigma_{k} \cdot \nabla\right) u_{t}+\left(\nabla \sigma_{k}\right) u_{t}\right] \dot{z}_{t}^{k}, \\
& \nabla p_{t}+Q\left[\left(u_{t} \cdot \nabla\right) u_{t}\right]=Q\left[\left(\sigma_{k} \cdot \nabla\right) u_{t}+\left(\nabla \sigma_{k}\right) u_{t}\right] \dot{z}_{t}^{k},
\end{aligned}
$$

where we note that $P \Delta u_{t}=\Delta P u_{t}=\Delta u_{t}$ when $\nabla \cdot u_{t}=0$. As usual, we study the equation for $u$ and later show that we can give meaning to the equation for $\nabla p$, see Lemma 3.8 and Remark 3.9. To this end, let us assume that $z^{k}$ is smooth and iterate the equation for $u$ into $\dot{z}_{t}^{k}$-term twice to obtain

$$
\delta u_{s t}+\int_{s}^{t} P\left[\left(u_{r} \cdot \nabla\right) u_{r}\right] d r=\int_{s}^{t} \vartheta \Delta u_{r} d r+\left[\mathcal{A}_{s t}^{1}+\mathcal{A}_{s t}^{2}\right] u_{s}+u_{s t}^{\natural},
$$

where we have defined

$$
\begin{aligned}
\mathcal{A}_{s t}^{1} \phi & :=P \tilde{\mathfrak{E}}_{\sigma_{k}} \phi Z_{s t}^{k}, \quad \mathcal{A}_{s t}^{2} \phi:=P \tilde{\mathfrak{£}}_{\sigma_{k}} P \tilde{\mathfrak{£}}_{\sigma_{l}} \phi \mathbb{Z}_{s t}^{l, k}, \quad \tilde{\mathfrak{E}}_{\sigma_{k}} \phi:=\left(\sigma_{k} \cdot \nabla\right) \phi+\left(\nabla \sigma_{k}\right) \phi, \\
u_{s t}^{\natural} & :=\int_{s}^{t} P \tilde{\mathfrak{£}}_{\sigma_{k}} \delta \mu_{s r} d z_{r}^{k}+\int_{s}^{t} P \tilde{\mathfrak{E}}_{\sigma_{k}} \int_{s}^{r} P \tilde{\mathfrak{E}}_{\sigma_{l}}\left(\delta \mu_{s r_{1}}+P \int_{s}^{r_{1}} \tilde{\mathfrak{E}}_{\sigma_{m}} u_{r_{2}} d z_{r_{2}}^{m}\right) d z_{r_{1}}^{l} d z_{r}^{k},
\end{aligned}
$$

and

$$
\mu_{t}=\int_{0}^{t}\left[\vartheta \Delta u_{r}-P\left(u_{r} \cdot \nabla\right) u_{r}\right] d r
$$

In the above, we have used suggestive notation for the operator $\tilde{£}_{\sigma_{k}}$ as a reminder that it related to the Lie derivative. Since $u$ is a vector field, the Lie-derivative of $u$ by $\sigma_{k}$ is given by $\mathfrak{f}_{\sigma_{k}} u:=\left(\sigma_{k} \cdot \nabla\right) \phi-(u \cdot \nabla) \sigma_{k}$, which is not that same as $\tilde{\mathfrak{f}}_{\sigma_{k}} u$ unless $\sigma_{k}$ is constant in space. However, the Lie derivative of the one-form $u^{b}=\delta_{i j} u^{j} d x^{i}$ by $\sigma_{k}$ is given by

$$
£_{\sigma_{k}} u^{b}=\left(\sigma_{k}^{j} \partial_{j} u^{i}+\partial_{i} \sigma_{k}^{j} u^{j}\right) d x^{i}
$$

and hence $\tilde{\mathfrak{E}}_{\sigma_{k}} u=\left(\mathfrak{f}_{\sigma_{k}} u^{b}\right)^{\sharp}$, where we recall that $\sharp$ is the inverse of $b$ as introdued in Section 1.2

Since we are concerned with strong solutions, we expect $u \in L_{T}^{\infty} \mathbf{H}^{1}$ and $\mu \in C^{1-\operatorname{var}}([0, T]$; $\left.\mathbf{H}^{0}\right)$, and hence the remainder $u^{\natural}$ in (3.5) is expected to belong to $C_{2}^{\zeta \text {-var }}\left([0, T] ; \mathbf{H}^{-2}\right)$, for some $\zeta<1$. Assume now that $z^{k}$ is not a smooth path, but we know how to make sense of $\mathbb{Z}$. Then, the only term that lacks a priori meaning in (3.3) is the term $u^{\natural}$. However, from formal power counting of the integrals in (3.5), we still expect this term to be a negligible remainder provided $\sigma \in\left(\mathbf{W}_{\operatorname{div}}^{3, \infty}\right)^{K}$. Thus, equation (3.3) is to be understood in the sense that we define the remainder term $u^{\natural}$ from the solution $u$. This will be made precise in Definition 3.1 below.

The pair $\mathcal{A}=\left(\mathcal{A}^{1}, \mathcal{A}^{2}\right)$ is an unbounded $p$-rough driver in the sense of Definition 2.4 on the scale $\left(\mathbf{H}^{n}\right)_{n}$. Indeed, the existence of a control $\omega_{\mathcal{A}}$ such that (2.7) holds follows from (2.2) and the fact that $(Z, \mathbb{Z})$ is a $p$-rough path in the sense of Definition 2.3, which implies Chen's relation (2.8). We note that control $\omega_{\mathcal{A}}$ can be chosen to satisfy

$$
\omega_{\mathcal{A}}(s, t) \lesssim|\sigma|_{3, \infty} \omega_{Z}(s, t) \quad \forall(s, t) \in \Delta_{T}
$$

In fact, owing to (2.2), we have more than what (2.7) strictly requires. We use these in the proof of Lemma 4.1.

We will now give our first definition of a solution to (3.1). 
DEFINITION 3.1. We say that $u$ is a strong solution of (3.1) up to time $T^{*}$ if $u$ : $\left[0, T^{*}\right] \rightarrow \mathbf{H}^{1}$ is weakly continuous, $u \in L_{T^{*}}^{2} \mathbf{H}^{2} \cap L_{T^{*}}^{\infty} \mathbf{H}^{1}$ and $u^{\natural}: \Delta_{T^{*}} \rightarrow \mathbf{H}^{-2}$ defined by

$$
u_{s t}^{\natural}(\phi):=\delta u_{s t}(\phi)+\int_{s}^{t}\left[-\vartheta\left(\Delta u_{r}, \phi\right)+B_{P}\left(u_{r}\right)(\phi)\right] d r-u_{s}\left(\left[\mathcal{A}_{s t}^{1, *}+\mathcal{A}_{s t}^{2, *}\right] \phi\right),
$$

for all $\phi \in \mathbf{H}^{2}$ satisfies $u^{\natural} \in C_{2, \omega_{Z}, L}^{\frac{p}{3} \text {-var }}\left(\left[0, T^{*}\right] ; \mathbf{H}^{-2}\right)$ for some $L>0$.

REMARK 3.2. By the regularity assumption on $u$, it follows that the $d r$-integral in (3.7) is well defined.

REMARK 3.3. It is possible to formulate a rough version of (3.1) without projecting the equation onto the scale of divergence-free spaces by keeping the pressure in the equation. This formulation was discussed in [21] for the case of an energy conservative noise, but the computations carry over mutatis mutandis to the current case giving an equivalent formulation of (3.3) and (3.14) below.

REMARK 3.4. The expansion $\mathcal{A}_{s t}^{1} u_{s}+\mathcal{A}_{s t}^{2} u_{s}+u_{s t}^{\natural}$ should be thought of as the projection of the rough path integral; that is,

$$
\mathcal{A}_{s t}^{1} u_{s}+\mathcal{A}_{s t}^{2} u_{s}+u_{s t}^{\natural}=P \int_{s}^{t}\left[\left(\sigma_{k} \cdot \nabla\right)+\left(\nabla \sigma_{k}\right)\right] u_{r} d Z_{r}^{k}
$$

Indeed, the expression $\mathcal{A}_{s t}^{1} u_{s}+\mathcal{A}_{s t}^{2} u_{s}$ represents a local expansion of the rough path integral. If $u^{\natural}$ is a remainder, then by the sewing lemma, [21], Lemma B.1, this uniquely defines a path representing the rough integral.

3.1. Vorticity formulation. Applying the curl operator $\nabla \times \cdot$ to (3.1), we obtain

$$
\partial_{t} \xi+(u \cdot \nabla) \xi-\mathbf{1}_{d=3}(\xi \cdot \nabla) u=\vartheta \Delta \xi+\left[\left(\sigma_{k} \cdot \nabla\right) \xi-\mathbf{1}_{d=3}(\xi \cdot \nabla) \sigma_{k}\right] \dot{z}_{t}^{k} .
$$

Let us suppose that there exists a strong solution $u$ of (3.1) on $[0, T]$ as defined by Definition 3.1. In order to find a rough version of the vorticity formulation, we apply the curl operator $\nabla \times$ to both sides of (3.3). Using properties of the curl operator and that $\xi=\nabla \times u$ is a weakly continuous function $\xi:[0, T] \rightarrow \dot{\mathbf{H}}^{0}$ with $\xi \in L_{T}^{2} \dot{\mathbf{H}}^{1} \cap L_{T}^{\infty} \dot{\mathbf{H}}^{0}$, we find that $\xi^{\natural}: \Delta_{T} \rightarrow \dot{\mathbf{H}}^{-3}$ defined for all $\phi \in \dot{\mathbf{H}}^{3}$ and $(s, t) \in \Delta_{T}$ by

$$
\begin{aligned}
\xi_{s t}^{\natural}(\phi)= & \left.\delta \xi_{s t}(\phi)+\int_{s}^{t}\left[\vartheta\left(\nabla \xi_{r}, \nabla \phi\right)+\left(u_{r} \cdot \nabla\right) \xi_{r}\right)(\phi)-\mathbf{1}_{d=3}\left(\left(u_{r} \cdot \nabla\right) \xi_{r}\right)(\phi)\right] d r \\
& -\xi_{s}\left(\left[A_{s t}^{1, *}+A_{s t}^{2, *}\right] \phi\right),
\end{aligned}
$$

satisfies $\xi^{\natural} \in C_{2, \omega_{Z}, L}^{\frac{p}{3} \text {-var }}\left([0, T] ; \dot{\mathbf{H}}^{-3}\right)$ for some $L>0$, where $A^{1}$ and $A^{2}$ are defined by

$$
A_{s t}^{1} \phi=\nabla \times \mathcal{A}_{s t}^{1} \phi=\mathfrak{f}_{\sigma_{k}} \phi Z_{s t}^{k}=\left(\left(\sigma_{k} \cdot \nabla\right) \phi-\mathbf{1}_{d=3}(\phi \cdot \nabla) \sigma_{k}\right) Z_{s t}^{k}
$$

and

$$
\begin{aligned}
A_{s t}^{2} \phi= & \nabla \times \mathcal{A}_{s t}^{2} \phi=£_{\sigma_{k}} \mathfrak{\sigma}_{\sigma_{l}} \phi \mathbb{Z}_{s t}^{l, k} \\
= & \left(\left(\sigma_{k} \cdot \nabla\right)\left(\sigma_{l} \cdot \nabla\right) \phi-\mathbf{1}_{d=3}\left(\sigma_{k} \cdot \nabla\right)\left((\phi \cdot \nabla) \sigma_{l}\right)\right. \\
& \left.-\mathbf{1}_{d=3}\left(\left(\sigma_{l} \cdot \nabla\right) \phi \cdot \nabla\right) \sigma_{k}+\mathbf{1}_{d=3}\left(\left((\phi \cdot \nabla) \sigma_{l}\right) \cdot \nabla\right) \sigma_{k}\right) \mathbb{Z}_{s t}^{l, k} .
\end{aligned}
$$

Indeed, the equalities (3.9) and (3.10) follows from the fact that $\nabla \times P=\nabla \times$ and $\nabla \times \tilde{\mathfrak{E}}_{\sigma_{k}}=$ $\mathfrak{f}_{\sigma_{k}} \nabla \times$ on divergence-free vector fields, which can be checked by direct calculation or by 
appealing to the differential geometry (see page 451 in [31] or Appendix A.6. of [4]). We note that in dimension two, $\xi$ is a mean-free scalar and the equation preserves the mean, which is zero. Moreover, in dimension three, $\xi$ is a mean-free and divergence-free vector and the equation preserves mean and divergence-freeness.

It is clear that $\mathbf{A}=\left(A^{1}, A^{2}\right)$ satisfies (2.7) for the scale $\left(\dot{\mathbf{H}}^{n}\right)_{n}$ with a control $\omega_{A}$ by $(2.1)$. The control of $\omega_{A}$ can be chosen so that

$$
\omega_{A}(s, t) \lesssim|\sigma|_{3, \infty} \omega_{Z}(s, t) \quad \forall(s, t) \in \Delta_{T} .
$$

The inverse of the curl (i.e., the Biot-Savart operator $\mathcal{K}$ ) can only recover the mean-free part of a vector field. Thus, in order to be able to recover $u$ from $\xi$, we need to control the mean of $u$ in terms of $\xi$. We denote by $\bar{u}$ the spatial mean of $u$; that is, $\bar{u}$ is the $d$-dimensional vector with the $m$ th component given by $\bar{u}^{m}:=\left(u, e_{m}\right)$ where $e_{m}$ is the $m$ th basis vector of $\mathbf{R}^{d}$. Furthermore, let $v=u-\bar{u}$ be the mean-free part. The remainder of the mean satisfies

$$
\begin{aligned}
\bar{u}_{s t}^{\natural, m} & :=u_{s t}^{\natural}\left(e_{m}\right)=\left(\delta u_{s t}, e_{m}\right)-\left(\left[\mathcal{A}_{s t}^{1}+\mathcal{A}_{s t}^{2}\right] u_{s}, e_{m}\right) \\
& =\delta \bar{u}_{s t}^{m}-\left(\left[\mathcal{A}_{s t}^{1}+\mathcal{A}_{s t}^{2}\right] \bar{u}_{s}, e_{m}\right)-\left(\left[\mathcal{A}_{s t}^{1}+\mathcal{A}_{s t}^{2}\right] v_{s}, e_{m}\right) .
\end{aligned}
$$

Because $\bar{u}_{s}$ is constant in space, we find that $\left(\mathcal{A}_{s t}^{i} \bar{u}_{s}, e_{m}\right)=0$. Moreover, using that all vector fields under consideration are divergence free, we obtain

$$
\left(\mathcal{A}_{s t}^{1} v_{s}, e_{m}\right)=\left(P\left[\left(\sigma_{k} \cdot \nabla\right) v_{s}\right], e_{m}\right) Z_{s t}^{k}+\left(P\left[\left(\nabla \sigma_{k}\right) v_{s}\right], e_{m}\right) Z_{s t}^{k}=\left(v_{s}^{l}, \partial_{m} \sigma_{k}^{l}\right) Z_{s t}^{k}
$$

and

$$
\begin{aligned}
\left(\mathcal{A}_{s t}^{2} v_{s}, e_{m}\right)= & \left(P\left(\left(\sigma_{k} \cdot \nabla\right) P\left[\sigma_{j} \cdot \nabla v_{s}\right]\right), e_{m}\right) \mathbb{Z}_{s t}^{j, k}+\left(P\left(\left(\sigma_{k} \cdot \nabla\right) P\left[\left(\nabla \sigma_{j}\right) v_{s}\right]\right), e_{m}\right) \mathbb{Z}_{s t}^{j, k} \\
& \left.\left.+\left(P\left(\left(\nabla \sigma_{k}\right) P\left[\left(\sigma_{j} \cdot \nabla\right) v_{s}\right]\right)\right), e_{m}\right) \mathbb{Z}_{s t}^{j, k}+\left(P\left(\left(\nabla \sigma_{k}\right) P\left[\left(\nabla \sigma_{j}\right) v_{s}\right]\right)\right), e_{m}\right) \mathbb{Z}_{s t}^{j, k} \\
= & \left.\left.\left(P\left(\left(\nabla \sigma_{k}\right) P\left[\left(\sigma_{j} \cdot \nabla\right) v_{s}\right]\right)\right), e_{m}\right) \mathbb{Z}_{s t}^{j, k}+\left(P\left(\left(\nabla \sigma_{k}\right) P\left[\left(\nabla \sigma_{j}\right) v_{s}\right]\right)\right), e_{m}\right) \mathbb{Z}_{s t}^{j, k} .
\end{aligned}
$$

For the remaining terms, we write

$$
\begin{aligned}
\left(P\left(\left(\nabla \sigma_{k}\right) P\left[\left(\sigma_{j} \cdot \nabla\right) v_{s}\right]\right), e_{m}\right) & =\left(P\left[\left(\sigma_{j} \cdot \nabla\right) v_{s}\right], \partial_{m} \sigma_{k}\right) \\
& =\left(\left(\sigma_{j} \cdot \nabla\right) v_{s}, \partial_{m} \sigma_{k}\right)=-\left(v_{s}^{l}, \partial_{i}\left(\sigma_{j}^{i} \partial_{m} \sigma_{k}^{l}\right)\right)
\end{aligned}
$$

and

$$
\left(P\left(\left(\nabla \sigma_{k}\right) P\left[\nabla \sigma_{j} v_{s}\right]\right), e_{m}\right)=\left(P\left[\nabla \sigma_{j} v_{s}\right], \partial_{m} \sigma_{k}\right)=\left(\nabla \sigma_{j} v_{s}, \partial_{m} \sigma_{k}\right) .
$$

Consequently, we get that

$$
\bar{u}_{s t}^{\natural}=\delta \bar{u}_{s t}-L_{s t}^{1}\left(v_{s}\right)-L_{s t}^{2}\left(v_{s}\right),
$$

where the $m$ th component of $L_{s t}^{1}\left(v_{s}\right)$ and $L_{s t}^{2}\left(v_{s}\right)$ is given by

$$
\left(v_{s}^{l}, \partial_{m} \sigma_{k}^{l}\right) Z_{s t}^{k} \quad \text { and } \quad\left(v_{s}^{l}, \partial_{n} \sigma_{j}^{l} \partial_{m} \sigma_{k}^{n}-\sigma_{j}^{n} \partial_{n} \partial_{m} \sigma_{k}^{l}\right) \mathbb{Z}_{s t}^{j, k}
$$

respectively. It follows that

$$
\left|L_{s t}^{1}\left(v_{s}\right)\right| \lesssim|\sigma|_{3, \infty}\left|v_{s}\right|_{0} \omega_{Z}(s, t)^{\frac{1}{p}} \quad \text { and } \quad\left|L_{s t}^{2}\left(v_{s}\right)\right| \lesssim|\sigma|_{3, \infty}\left|v_{s}\right|_{0} \omega_{Z}(s, t)^{\frac{2}{p}}
$$

DEFINITION 3.5. We say that a pair $(\xi, \bar{u})$ is a weak solution of (3.8) up to time $T^{*}$ if $(\xi, \bar{u}):\left[0, T^{*}\right] \rightarrow \dot{\mathbf{H}}^{0} \times \mathbf{R}^{d}$ is weakly continuous, $\xi \in L_{T^{*}}^{2} \dot{\mathbf{H}}^{1} \cap L_{T^{*}}^{\infty} \dot{\mathbf{H}}^{0}$, and $\bar{u} \curvearrowleft: \Delta_{T^{*}} \rightarrow \mathbf{R}^{d}$ and $\xi^{\natural}: \Delta_{T^{*}} \rightarrow \dot{\mathbf{H}}^{-3}$ defined by

$$
\begin{aligned}
\bar{u}_{s t}^{\natural}:= & \delta \bar{u}_{s t}-\left[L_{s t}^{1}+L_{s t}^{2}\right]\left(\mathcal{K} \xi_{s}\right), \\
\xi_{s t}^{\natural}(\phi):= & \delta \xi_{s t}(\phi)+\int_{s}^{t}\left[\vartheta\left(\nabla \xi_{r}, \nabla \phi\right)+\left(\left[\left(\mathcal{K} \xi_{r}+\bar{u}_{r}\right) \cdot \nabla\right] \xi_{r}, \phi\right)\right. \\
& \left.\quad-\mathbf{1}_{d=3}\left(\left[\xi_{r} \cdot \nabla\right] \mathcal{K} \xi_{r}, \phi\right)\right] d r-\xi_{s}\left(\left[A_{s t}^{1, *}+A_{s t}^{2, *}\right] \phi\right),
\end{aligned}
$$


for all $\phi \in \dot{\mathbf{H}}^{3}$ satisfy $\xi^{\natural} \in C_{2, \omega_{Z}, L}^{\frac{p}{3} \text {-var }}\left(\left[0, T^{*}\right] ; \dot{\mathbf{H}}^{-3}\right)$ and $\bar{u}^{\natural} \in C_{2, \omega_{Z}, L}^{\frac{p}{3} \text {-var }}\left(\left[0, T^{*}\right] ; \mathbf{R}^{d}\right)$ for some $L>0$.

LEMMA 3.6. There is a one-to-one correspondence between the solutions defined in Definitions 3.1 and 3.5 .

PROOF. If $u$ is a strong solution of (3.1), then by defining

$$
\xi=\nabla \times u, \quad \xi^{\natural}=\nabla \times u^{\natural}, \quad \bar{u}^{\natural}=\left(u^{\natural}\left(e_{m}\right)\right)_{m=1}^{d},
$$

we obtain a solution in the sense of Definition 3.5. For the reverse direction, define

$$
v=\mathcal{K} \xi, \quad v^{\natural}=\mathcal{K} \xi^{\natural}, \quad u=\bar{u}+v, \quad u^{\natural}=v^{\natural}+\bar{u}^{\natural} .
$$

Because $\mathcal{K}$ is linear and commutes with derivatives, we have

$$
\delta v=\mathcal{K} \delta \xi, \quad \Delta v=\mathcal{K} \Delta \xi, \quad(\bar{u} \cdot \nabla) v=\mathcal{K}[(\bar{u} \cdot \nabla) \xi]
$$

By direct calculation we obtain

$$
\operatorname{curl}(P[(v \cdot \nabla) v])=v \cdot \nabla \xi-\mathbf{1}_{d=3}(\nabla v) \xi
$$

and

$$
\operatorname{curl}\left(\left[\mathcal{A}_{s t}^{1}+\mathcal{A}_{s t}^{2}\right] u-\left[L_{s t}^{1}+L_{s t}^{2}\right](v)\right)=\left[A_{s t}^{1}+A_{s t}^{2}\right] \xi
$$

where we emphasize that both arguments of the curl are divergence and mean-free. Thus, using that $\mathcal{K}$ o curl is the identity on the space of divergence and mean-free test functions, we get

$$
\begin{aligned}
& P[(v \cdot \nabla) v]=\mathcal{K}\left[(v \cdot \nabla) \xi-\mathbf{1}_{d=3}(\nabla v) \xi\right] \\
& {\left[\mathcal{A}_{s t}^{1}+\mathcal{A}_{s t}^{2}\right] u-\left[L_{s t}^{1}+L_{s t}^{2}\right](v)=\mathcal{K}\left(\left[A_{s t}^{1}+A_{s t}^{2}\right] \xi\right)}
\end{aligned}
$$

Applying $\mathcal{K}$ to the vorticity equation (3.12), we find that for all $\phi \in \mathbf{H}^{3}$ and $(s, t) \in \Delta_{T}$,

$$
\begin{aligned}
v_{s t}^{\natural}(\phi):= & \delta v_{s t}(\phi)+\int_{s}^{t}\left[\vartheta\left(\nabla v_{r}, \nabla \phi\right)+B_{P}\left(v_{r}+\bar{u}_{r}, v_{r}\right)(\phi)\right] d r \\
& -u_{s}\left(\left[\mathcal{A}_{s t}^{1, *}+\mathcal{A}_{s t}^{2, *}\right] \phi\right)+\left[L_{s t}^{1}+L_{s t}^{2}\right]\left(v_{s}\right)(\phi) .
\end{aligned}
$$

Therefore, for all $\phi \in \mathbf{H}^{3}$ and $(s, t) \in \Delta_{T}$,

$$
u_{s t}^{\natural}(\phi)=\delta u_{s t}(\phi)+\int_{s}^{t}\left[\vartheta\left(\nabla u_{r}, \nabla \phi\right)+B_{P}\left(u_{r}, u_{r}\right)(\phi)\right] d r-u_{s}\left(\left[\mathcal{A}_{s t}^{1, *}+\mathcal{A}_{s t}^{2, *}\right] \phi\right),
$$

which completes the proof.

3.2. Main results. Our main results concern existence and uniqueness of strong solutions, stability with respect to the given data including the driving signal, and the existence of a random dynamical system generated by the solution. Let us begin with the precise formulation of the existence result. 


\subsubsection{Existence and uniqueness.}

TheOREM 3.7. Let $d \in\{2,3\}$. For a given $u_{0} \in \mathbf{H}^{1}, \mathbf{Z} \in \mathcal{C}_{g}^{p \text {-var }}\left([0, T] ; \mathbf{R}^{K}\right)$, and $\sigma \in$ $\left(\mathbf{W}_{\mathrm{div}}^{3, \infty}\right)^{K}$, there exists a time $T^{*}$ depending only on $p, \omega_{Z},|\sigma|_{3, \infty}$ and $\left|u_{0}\right|_{1}$, and a strong solution up to time $T^{*}$ of (3.1) satisfying the energy inequality

$$
\sup _{t \in\left[0, T^{*}\right]}\left|u_{t}\right|_{1}^{2}+\int_{0}^{T^{*}}\left|\nabla^{2} u_{r}\right|_{0}^{2} d r \leq F\left(\left|u_{0}\right|_{1}\right),
$$

for some continuous function $F: \mathbf{R}_{+} \rightarrow \mathbf{R}_{+}$. Moreover, $u \in C^{p-\operatorname{var}}\left(\left[0, T^{*}\right] ; \mathbf{H}^{0}\right)$, and if $d=2$, the final time $T^{*}$ can be taken to be $T$.

The proof of existence of a solution is a consequence of the stronger statement in Theorem 6.1 presented in Section 6. The theorem is based on a suitable Galerkin approximation, combined with an approximation of the driving signal $z$ by smooth paths. The fact that $u \in C^{p \text {-var }}\left(\left[0, T^{*}\right] ; \mathbf{H}^{0}\right)$ follows from the a priori result in Lemma 4.3.

The next result shows how to construct the pressure from the velocity field. The proof of this statement can be found in Section 4.

LEMMA 3.8. Working under the same assumptions as Theorem 3.7, if $u$ is a strong solution of (3.1) up to time $T^{*}$, the pressure $\pi$ can be recovered. More precisely, there exists $\pi \in C^{p-\operatorname{var}}\left(\left[0, T^{*}\right] ; \mathbf{H}_{\perp}^{-2}\right)$ satisfying

$$
\left.\delta \pi_{s t}+\int_{s}^{t} Q\left[\left(u_{r} \cdot \nabla\right) u_{r}\right)\right] d r=\left[\mathcal{A}_{s t}^{Q, 1}+\mathcal{A}_{s t}^{Q, 2}\right] u_{s}+u_{s t}^{Q, \natural},
$$

where

$$
\begin{aligned}
\mathcal{A}_{s t}^{Q, 1} \phi & :=Q\left[\left(\sigma_{k} \cdot \nabla+\nabla \sigma_{k}\right) \phi\right] Z_{s t}^{k}, \\
\mathcal{A}_{s t}^{Q, 2} \phi & :=Q\left[\left(\sigma_{k} \cdot \nabla+\nabla \sigma_{k}\right) P\left[\left(\sigma_{l} \cdot \nabla+\nabla \sigma_{l}\right) \phi\right]\right] \mathbb{Z}_{s t}^{l, k},
\end{aligned}
$$

and $u^{Q, \natural} \in C_{2, \varpi, \tilde{L}}^{\frac{3}{p}-v a r}\left(\left[0, T^{*}\right] ; \mathbf{H}_{\perp}^{-2}\right)$ for $\varpi(s, t)=t-s+\omega_{Z}(s, t)$ and some $\tilde{L}>0$.

REMARK 3.9. In the lemma above, as in Remark 3.4, we note that $\left[\mathcal{A}_{s t}^{Q, 1}+\mathcal{A}_{s t}^{Q, 2}\right] u_{s}+$ $u_{s t}^{Q, \natural}$ is a rough integral $Q \int_{s}^{t}\left(\sigma_{k} \cdot \nabla+\nabla \sigma_{k}\right) u_{r} d z_{r}^{k}$. Thus, adding $u$ and $\pi$ and using that $P+Q=I$ gives that

$$
\delta u_{s t}+\delta \pi_{s t}=\int_{s}^{t}\left[\vartheta \Delta u_{r}-\left(u_{r} \cdot \nabla\right) u_{r}\right] d r+\int_{s}^{t}\left(\sigma_{k} \cdot \nabla+\nabla \sigma_{k}\right) u_{r} d z_{r}^{k}
$$

We also remark that the pair $\left(\mathcal{A}^{Q, 1}, \mathcal{A}^{Q, 2}\right)$ is, in general, not an unbounded rough driver on the scale $\left(\mathbf{H}_{\perp}^{n}\right)_{n}$, because it fails to satisfy Chen's relation (2.8). Nevertheless, we have

$$
\delta \mathcal{A}_{s \theta t}^{Q, 2}=\mathcal{A}_{\theta t}^{Q, 1} \mathcal{A}_{s \theta}^{1} \quad \text { for all }(s, \theta, t) \in \Delta_{T}^{(2)},
$$

which is the correct Chen's relation for the system of equations (3.3) and (3.14) needed to recover the pressure from $u$ (see the proof of Lemma 3.8).

In dimension two, we obtain classical enstrophy balance and uniqueness. 
THEOREM 3.10. In dimension two, for a given $u_{0} \in \mathbf{H}^{1}$ and $\mathbf{Z} \in \mathcal{C}_{g}^{p \text {-var }}\left([0, T] ; \mathbf{R}^{K}\right)$ and $\sigma \in\left(\mathbf{W}_{\text {div }}^{3, \infty}\right)^{K}$, there is at most one strong solution to (3.1). Moreover, the velocity $u$ belongs to $C_{T} \mathbf{H}^{1}$, the following enstrophy balance holds

$$
\sup _{t \in[0, T]}\left|\xi_{t}\right|_{0}^{2}+2 \vartheta \int_{0}^{T}\left|\nabla \xi_{r}\right|_{0}^{2} d r=\left|\xi_{0}\right|_{0}^{2}
$$

and the velocity satisfies the energy estimate (3.13) for any $T^{*} \leq T$.

Theorem 3.10 will follow from Theorem 5.6 and Theorem 5.10 presented in Section 5.

REMARK 3.11. Except in the case when the $\sigma_{k}, k \in\{1, \ldots, K\}$ are all constant in space, there is no reason to believe that one could obtain energy equality for the velocity $u$, since the multiplicative term $\left(\nabla \sigma_{k}\right) u \dot{z}^{k}$ will add or subtract energy to the system.

3.2.2. Stability. Owing to Theorem 3.7 and Theorem 3.10, in dimension two, there exists a solution map $\Gamma$ that maps every initial condition $u_{0} \in \mathbf{H}^{1}$, continuous geometric $p$-rough path $\mathbf{Z}=(Z, \mathbb{Z})$, and family of divergence-free vector fields $\sigma \in\left(\mathbf{W}_{\text {div }}^{3, \infty}\right)^{K}$, to a unique strong solution $u$ of (3.1). Let us denote by $\mathbf{H}_{w}^{1}$ the space $\mathbf{H}^{1}$ equipped with its weak topology. The following stability result is proved in Section 5.3.

COROLlary 3.12. In dimension two, the solution map

$$
\begin{gathered}
\Gamma: \mathbf{H}^{1} \times\left(\mathbf{W}_{\mathrm{div}}^{3, \infty}\right)^{K} \times \mathcal{C}_{g}^{p-\operatorname{var}}\left([0, T] ; \mathbf{R}^{K}\right) \rightarrow L_{T}^{2} \mathbf{H}^{1} \cap C_{T} \mathbf{H}_{w}^{1} \cap C_{T} \mathbf{H}^{0} \\
\left(u_{0}, \sigma, \mathbf{Z}\right) \mapsto u
\end{gathered}
$$

is continuous.

In particular, the following Wong-Zakai result holds true. Let $\left\{B^{n}\right\}$ be a piecewise linear interpolation of a Brownian motion $B$, and for each $n$, denote by $u^{n}$ the unique strong solution of (3.1) with $\dot{z}$ is replaced by $\dot{B}^{n}$, existence of which is guaranteed by Theorem 3.10 and Theorem 3.7. Then $\left\{u^{n}\right\}$ converges almost surely to $u$ in $L_{T}^{2} \mathbf{H}^{1} \cap C_{T} \mathbf{H}_{w}^{1} \cap C_{T} \mathbf{H}^{0}$ where $u$ is the strong probabilistic, pathwise unique solution of

$$
\begin{aligned}
d u_{t}+\nabla d p_{t} & =\left[\vartheta \Delta u_{t}-\left(u_{t} \cdot \nabla\right) u_{t}\right] d t+\left[\left(\sigma_{k} \cdot \nabla\right)+\left(\nabla \sigma_{k}\right)\right] u_{t} \circ d B_{t}^{k}, \\
\nabla \cdot u_{t} & =0,\left.\quad u_{t}\right|_{t=0}=u_{0} \in \mathbf{H}^{1}
\end{aligned}
$$

constructed in [29], Theorem 2.1, for the more general case of $u_{0} \in \mathbf{H}^{0}$, where $d p=\tilde{p} d t+$ $q_{k} \circ d B_{t}^{k}$ is the pressure semimartingale.

Moreover, the energy estimate (3.13) is satisfied for solutions corresponding to almost all sample paths of the Brownian motion.

REMARK 3.13. By applying the curl operator to $u$, we also obtain continuity of the mapping

$$
\begin{aligned}
\Gamma: \mathbf{H}^{1} \times\left(\mathbf{W}_{\mathrm{div}}^{3, \infty}\right)^{K} \times \mathcal{C}_{g}^{p-\operatorname{var}}\left([0, T] ; \mathbf{R}^{K}\right) & \rightarrow L_{T}^{2} \dot{L}^{2} \cap C_{T} \dot{L}_{w}^{2} \cap C_{T} \dot{W}^{-1,2}, \\
\left(u_{0}, \sigma, \mathbf{Z}\right) & \mapsto \xi .
\end{aligned}
$$


3.2.3. Random dynamical system. Based on our well-posedness and stability result in dimension two, under suitable assumptions on the driving rough path, we are able to construct a continuous random dynamical system corresponding to the Navier-Stokes equations (3.1).

Let us first introduce the necessary definitions. Let $(\Omega, \mathcal{F})$ and $(X, \mathcal{B})$ be measurable spaces. A family $\theta=\left(\theta_{t}\right)_{t \geq 0}$ of maps from $\Omega$ to itself is a measurable dynamical system provided:

1. $(t, \omega) \mapsto \theta_{t} \omega$ is measurable w.r.t. the $\sigma$-algebras $\mathcal{B}([0, \infty)) \otimes \mathcal{F}$ and $(\Omega, \mathcal{F})$,

2. $\theta_{0}=\mathrm{Id}$,

3. $\theta_{s+t}=\theta_{t} \circ \theta_{s}$ for all $s, t \geq 0$.

If $\mathbb{P}$ is a probability measure on $(\Omega, \mathcal{F})$ that is invariant under $\theta$, that is, $\mathbb{P} \circ \theta_{t}^{-1}=\mathbb{P}$ for all $t \geq 0$, we call $(\Omega, \mathcal{F}, \mathbb{P}, \theta)$ a measurable metric dynamical system. A measurable random dynamical system on $(X, \mathcal{B})$ is a measurable metric dynamical system $(\Omega, \mathcal{F}, \mathbb{P}, \theta)$ together with a measurable map $\varphi:[0, \infty) \times \Omega \times X \rightarrow X$ satisfying the cocycle property; that is, $\varphi_{0}(\omega)=\operatorname{Id}_{X}$ and

$$
\varphi_{s+t}(\omega)=\varphi_{t}\left(\theta_{s} \omega\right) \circ \varphi_{s}(\omega)
$$

for all $s, t \in[0, \infty)$ and $\omega \in \Omega$. If, in addition, $X$ is a topological space and the map $\varphi(\cdot, \omega, \cdot):[0, \infty) \times X \rightarrow X$ is continuous for every $\omega \in \Omega$, then it is called a continuous random dynamical system.

Under suitable assumptions on the coefficients, rough path driven differential equations generate random dynamical systems provided the driving rough path is a rough path cocycle [3]. To be more precise, if $p \in[2,3)$ and $(\Omega, \mathcal{F}, \mathbb{P}, \theta)$ is a measurable metric dynamical system, then we say that

$$
\mathbf{Z}=(Z, \mathbb{Z}): \Omega \rightarrow C_{2, \operatorname{loc}}^{p-\operatorname{var}}\left([0, \infty) ; \mathbf{R}^{K}\right) \times C_{2, \operatorname{loc}}^{p-\operatorname{var}}\left([0, \infty) ; \mathbf{R}^{K \times K}\right)
$$

is a continuous $p$-rough path cocycle provided $\left.\mathbf{Z}(\omega)\right|_{[0, T]}$ is a continuous $p$-rough path for every $T>0$ and $\omega \in \Omega$ and the following cocycle property

$$
Z_{s, s+t}(\omega)=Z_{t}\left(\theta_{s} \omega\right), \quad \mathbb{Z}_{s, s+t}(\omega)=\mathbb{Z}_{0, t}\left(\theta_{s} \omega\right),
$$

holds true for every $s, t \geq 0$ and $\omega \in \Omega$. Similarly, one may define a $p$-rough path cocycle for any $p \in[1, \infty)$. It was shown in Section 2 in [3] that rough path lifts of various stochastic processes define cocycles. These include Gaussian processes with stationary increments and independent components under certain assumption on the covariance, satisfied, for instance, by the fractional Brownian motion with Hurst parameter $H>1 / 4$.

COROLLARY 3.14. If the driving rough path $\mathbf{Z}$ is a continuous p-rough path cocycle for some $p \in[2,3)$, then, in dimension two, the system (3.1) generates a continuous random dynamical system on $\mathbf{H}^{1}$.

Proof. Let $\psi:[0, \infty) \times[0, \infty) \times \Omega \times \mathbf{H}^{1} \rightarrow \mathbf{H}^{1}$ be the random flow generated by (3.1); that is, $\psi\left(t, s, \omega, u_{0}\right)$ is the unique solution to (3.1) starting at time $s$ from the initial condition $u_{0}$, driven by $\mathbf{Z}(\omega)$, and evaluated at the time $t$. Then it follows from our definition of the solution and the cocycle property of $\mathbf{Z}$ that $\psi\left(t+h, s+h, \omega, u_{0}\right)=\psi\left(t, s, \theta_{h} \omega, u_{0}\right)$. Consequently, we define $\varphi\left(t, \omega, u_{0}\right)=\psi\left(t, 0, \omega, u_{0}\right)$ and using also the semiflow property of $\psi$, which follows from uniqueness, we deduce that $\varphi$ has the cocycle property. The continuity with respect to time and the initial condition follows from (5.8). 
4. A priori estimates. In this section, we derive a priori estimates of the remainder term

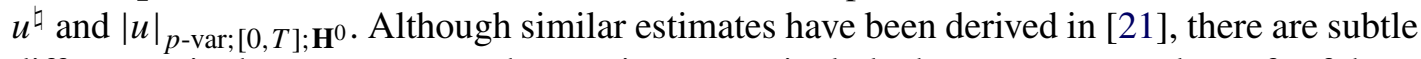
differences in the present paper that motivate us to include the statements and proofs of these estimates. Indeed, in [21], we were interested in weak solutions, and in the present paper, we are concerned with strong solutions. So, at the surface, the a priori estimates involve one extra derivative. However, in [21], we required a scale of fractional Sobolev spaces, and in the present paper, we are able to avoid this due to proved estimates on the nonlinearity, which stem from the fact that we are working with strong solutions.

Let $u$ be a solution of (3.1) up to time $T^{*}$ in the sense of Definition 3.1. We recall the definition of $\mu$ in (3.6) which we restrict to the scale $\left(\mathbf{H}^{n}\right)_{n}$. It follows that for all $(s, t) \in \Delta_{T^{*}}$,

$$
\delta u_{s t}=\delta \mu_{s t}+\mathcal{A}_{s t}^{1} u_{s}+\mathcal{A}_{s t}^{2} u_{s}+u_{s t}^{\natural},
$$

where the equality holds in $\mathbf{H}^{-2}$. Using (2.4) with $m_{1}, m_{3}=1$ and $m_{2}=0$ we obtain $\left|B_{P}\left(u_{r}\right)\right|_{-1} \lesssim\left|u_{r}\right|_{1}^{2}$, and hence for all $(s, t) \in \Delta_{T^{*}}$,

$$
\left|\delta \mu_{s t}\right|_{-1} \lesssim\left(1+|u|_{L_{T^{*}}^{\infty} \mathbf{H}^{1}}\right)^{2}(t-s) .
$$

The following intermediate remainder term will play an important role in the proof of the priori estimates:

$$
u_{s t}^{\sharp}:=\delta u_{s t}-\mathcal{A}_{s t}^{1} u_{s}=\delta \mu_{s t}+\mathcal{A}_{s t}^{2} u_{s}+u_{s t}^{\natural} .
$$

The first expression for the intermediate remainder has low regularity in time, but is not very irregular in space. On the other hand, the second expression has higher regularity in time than the first expression, but is less regular in space. We derive a priori estimates on the remainder $u^{\natural}$ using an interpolation argument with the help of the smoothing operators introduced in Definition 2.5.

In this section, for brevity, we denote $\|\cdot\|_{n, m}=|\cdot|_{\mathcal{L}\left(\mathbf{H}^{n}, \mathbf{H}^{m}\right)}$. The following result is similar as in [21], Lemma 3.1, but with the added spatial regularity of the solution, which allows us to circumvent the use of fractional Sobolev spaces as in [21].

LEMMA 4.1. Assume that $u$ is a solution of (3.1) up to time $T^{*}$. Then there is a positive constant $\tilde{L}=\tilde{L}\left(p,|\sigma|_{3, \infty}\right)<L$ such that for all $(s, t) \in \Delta_{T^{*}}$ with $\omega_{Z}(s, t) \leq \tilde{L}$,

$$
\begin{aligned}
\omega_{\natural}(s, t) & :=\left|u^{\natural}\right|_{\frac{p}{3}-\operatorname{var} ;[s, t] ; \mathbf{H}^{-2}}^{\frac{p}{3}} \\
& \lesssim p,|\sigma|_{3, \infty}|u|_{L_{T^{*}}^{\infty} \mathbf{H}^{1}}^{\frac{p}{3}} \omega_{Z}(s, t)+\left(1+|u|_{L_{T^{*}}^{\infty} \mathbf{H}^{1}}\right)^{\frac{2 p}{3}}(t-s)^{\frac{p}{3}} \omega_{Z}(s, t)^{\frac{1}{3}} .
\end{aligned}
$$

Proof. First, we apply $\delta$ to (3.7) and deduce that for all $\phi \in \mathbf{H}^{2}$ and $(s, \theta, t) \in \Delta_{T^{*}}$,

$$
\delta u_{s \theta t}^{\natural}(\phi)=\delta u_{s \theta}\left(\mathcal{A}_{\theta t}^{2, *} \phi\right)+u_{s \theta}^{\sharp}\left(\mathcal{A}_{\theta t}^{1, *} \phi\right),
$$

where $u_{s \theta}^{\sharp}$ is defined in (4.3). Second, we decompose $\delta u_{s \theta t}^{\natural}(\phi)$ into a smooth (in space) and nonsmooth part using the self-adjoint smoothing operator $J^{\eta}$ :

$$
\delta u_{s \theta t}^{\natural}(\phi)=\left(I-J^{\eta}\right) \delta u_{s \theta t}^{\natural}(\phi)+J^{\eta} \delta u_{s \theta t}^{\natural}(\phi),
$$

where $\eta \in(0,1]$ will be specified at the end of the proof. We will now use an interpolation argument to estimate each term using the two different expressions (4.3), and then invoke the sewing lemma. 
We use the self-adjointness of $J^{\eta}$, the decomposition $u_{s \theta}^{\sharp}=\delta u_{s \theta}-\mathcal{A}_{s \theta}^{1} u_{s}$, (2.2), and (2.9) to estimate the smooth part:

$$
\begin{aligned}
\left|\left(I-J^{\eta}\right) \delta u_{s \theta t}^{\natural}\right|_{-2} \lesssim & |u|_{L_{T^{*}}^{\infty} \mathbf{H}^{1}}\left(\left\|I-J^{\eta}\right\|_{0,-1}\left\|\mathcal{A}_{\theta t}^{2 *}\right\|_{2,0}+\left\|I-J^{\eta}\right\|_{1,-1}\left\|\mathcal{A}_{\theta t}^{1, *}\right\|_{2,1}\right. \\
& \left.+\left\|\mathcal{A}_{s \theta}^{1, *}\right\|_{0,-1}\left\|I-J^{\eta}\right\|_{1,0}\left\|\mathcal{A}_{\theta t}^{1, *}\right\|_{2,1}\right) \\
\lesssim & |u|_{L_{T^{*}}^{\infty} \mathbf{H}^{1}}\left(\omega_{Z}(s, t)^{\frac{2}{p}} \eta+\omega_{Z}(s, t)^{\frac{1}{p}} \eta^{2}\right) .
\end{aligned}
$$

In order to estimate the nonsmooth part, we use the decomposition $u_{s \theta}^{\sharp}=\delta \mu_{s \theta}+\mathcal{A}_{s \theta}^{2} u_{s}+u_{s \theta}^{\natural}$ :

$$
\begin{aligned}
J^{\eta} \delta u_{s \theta t}^{\natural}(\phi)= & \delta \mu_{s \theta}\left(J^{\eta} \mathcal{A}_{\theta t}^{2, *} \phi\right)+u_{s}\left(\mathcal{A}_{s \theta}^{1, *} J^{\eta} \mathcal{A}_{\theta t}^{2, *} \phi\right)+u_{s}\left(\mathcal{A}_{s \theta}^{2, *} J^{\eta} \mathcal{A}_{\theta t}^{2, *} \phi\right)+u_{s \theta}^{\natural}\left(J^{\eta} \mathcal{A}_{\theta t}^{2, *} \phi\right) \\
& +\delta \mu_{s \theta}\left(J^{\eta} \mathcal{A}_{\theta t}^{1, *} \phi\right)+u_{s}\left(\mathcal{A}_{s \theta}^{2, *} J^{\eta} \mathcal{A}_{\theta t}^{1, *} \phi\right)+u_{s \theta}^{\natural}\left(J^{\eta} \mathcal{A}_{\theta t}^{1, *} \phi\right) .
\end{aligned}
$$

For all $(s, \theta, t) \in \Delta_{T^{*}}^{(2)}$ such that $\omega_{Z}(s, t) \leq L$, we have

$$
\begin{aligned}
\left|J^{\eta} \delta u_{s \theta t}^{\natural}\right|_{-2} \leq & \left|\delta \mu_{s \theta}\right|_{-1}\left\|J^{\eta}\right\|_{0,1}\left\|\mathcal{A}_{\theta t}^{2, *}\right\|_{2,0}+|u|_{L_{T^{*}}^{\infty} \mathbf{H}^{1}}\left\|\mathcal{A}_{s \theta}^{1, *}\right\|_{0,-1}\left\|J^{\eta}\right\|_{0,0}\left\|\mathcal{A}_{\theta t}^{2, *}\right\|_{2,0} \\
& +|u|_{L_{T^{*}}^{\infty} \mathbf{H}^{1}}\left\|\mathcal{A}_{s \theta}^{2, *}\right\|_{1,-1}\left\|J^{\eta}\right\|_{0,1}\left\|\mathcal{A}_{\theta t}^{2, *}\right\|_{2,0} \\
& +\omega_{\natural}(s, t)^{\frac{3}{p}}\left\|J^{\eta}\right\|_{0,2}\left\|\mathcal{A}_{\theta t}^{2, *}\right\|_{2,0}+\left\|\delta \mu_{s \theta}\right\|_{-1}\left\|J^{\eta}\right\|_{1,1}\left\|\mathcal{A}_{\theta t}^{1, *}\right\|_{2,1} \\
+ & |u|_{L_{T^{*}}^{\infty} \mathbf{H}^{1}}\left\|\mathcal{A}_{s \theta}^{2, *}\right\|_{1,-1}\left\|J^{\eta}\right\|_{1,1}\left\|\mathcal{A}_{\theta t}^{1, *}\right\|_{2,1}+\omega_{\natural}(s, t)^{\frac{3}{p}}\left\|J^{\eta}\right\|_{1,2}\left\|\mathcal{A}_{\theta t}^{1, *}\right\|_{2,1},
\end{aligned}
$$

and hence using (2.2), (2.9) and (4.2), we find

$$
\begin{aligned}
& \left|J^{\eta} \delta u_{s \theta t}^{\natural}\right|_{-2} \lesssim\left(1+|u|_{L_{T^{*}}^{\infty} \mathbf{H}^{1}}\right)^{2}(t-s) \omega_{Z}(s, t)^{\frac{2}{p}} \eta^{-1} \\
& +|u|_{L_{T^{*}}^{\infty} \mathbf{H}^{1}} \omega_{Z}(s, t)^{\frac{3}{p}}+|u|_{L_{T^{*}}^{\infty} \mathbf{H}^{1} \omega_{Z}(s, t)^{\frac{4}{p}} \eta^{-1}} \\
& +\omega_{\natural}(s, t)^{\frac{3}{p}} \omega_{Z}(s, t)^{\frac{2}{p}} \eta^{-2}+\left(1+|u|_{L_{T^{*}}^{\infty} \mathbf{H}^{1}}\right)^{2}(t-s) \omega_{Z}(s, t)^{\frac{1}{p}} \\
& +|u|_{L_{T^{*}}^{\infty} \mathbf{H}^{1}} \omega_{Z}(s, t)^{\frac{3}{p}}+\omega_{\natural}(s, t)^{\frac{3}{p}} \omega_{Z}(s, t)^{\frac{1}{p}} \eta^{-1} .
\end{aligned}
$$

Setting $\eta=\omega_{Z}(s, t)^{\frac{1}{p}} \lambda$ for some $\lambda>0$ to be determined later and combining the above estimates, we obtain

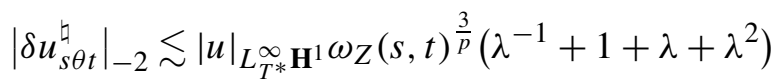

$$
\begin{aligned}
& +\left(1+|u|_{L_{T^{*}}^{\infty} \mathbf{H}^{1}}\right)^{2}(t-s) \omega_{Z}(s, t)^{\frac{1}{p}}\left(\lambda^{-1}+1\right)+\omega_{\natural}(s, t)^{\frac{3}{p}}\left(\lambda^{-1}+\lambda^{-2}\right) \\
& \lesssim\left(|u|_{L_{T^{*}}^{\infty} \mathbf{H}^{1}}^{\frac{p}{3}} \omega_{Z}(s, t)\left(\lambda^{-1}+1+\lambda+\lambda^{2}\right)^{\frac{p}{3}}\right. \\
& \left.+\left(1+|u|_{L_{T^{*}}^{\infty} \mathbf{H}^{1}}\right)^{\frac{2 p}{3}}(t-s)^{\frac{p}{3}} \omega_{Z}(s, t)^{\frac{1}{3}}\left(\lambda^{-1}+1\right)^{\frac{p}{3}}+\omega_{\natural}(s, t)\left(\lambda^{-1}+\lambda^{-2}\right)^{\frac{p}{3}}\right)^{\frac{3}{p}} .
\end{aligned}
$$

Applying the sewing lemma [21], Corollary B.2, we get

$$
\begin{aligned}
\left|u_{s t}^{\natural}\right|_{-2}^{\frac{p}{3}} \lesssim & |u|_{L_{T^{*}}^{\infty} \mathbf{H}^{1}}^{\frac{p}{3}} \omega_{Z}(s, t)\left(\lambda^{-1}+1+\lambda+\lambda^{2}\right)^{\frac{p}{3}} \\
& +\left(1+|u|_{L_{T^{*}}^{\infty} \mathbf{H}^{1}}\right)^{\frac{2 p}{3}}(t-s)^{\frac{p}{3}} \omega_{Z}(s, t)^{\frac{1}{3}}\left(\lambda^{-1}+1\right)^{\frac{p}{3}}+\omega_{\natural}(s, t)\left(\lambda^{-1}+\lambda^{-2}\right)^{\frac{p}{3}} .
\end{aligned}
$$


Since $\omega_{\natural}(s, t)=\left|u^{\natural}\right|_{\frac{p}{3}}^{\frac{p}{3}}-\operatorname{var} ;[s, t] ; \mathbf{H}^{-2}$ is equal to the infimum over all controls satisfying $\left|u_{s t}^{\natural}\right|_{-2} \leq \omega_{\natural}(s, t)^{\frac{3}{p}}$ (see $\left.(2.6)\right)$, there is a constant $C=C\left(p,|\sigma|_{3, \infty}\right)$ such that

$$
\begin{aligned}
\omega_{\natural}(s, t) \leq & C\left(|u|_{L_{T^{*}}^{\infty} \mathbf{H}^{1}}^{\frac{p}{3}} \omega_{Z}(s, t)\left(\lambda^{-1}+1+\lambda+\lambda^{2}\right)^{\frac{p}{3}}\right. \\
& \left.+\left(1+|u|_{L_{T^{*}}^{\infty} \mathbf{H}^{1}}\right)^{\frac{2 p}{3}}(t-s)^{\frac{p}{3}} \omega_{Z}(s, t)^{\frac{1}{3}}\left(\lambda^{-1}+1\right)^{\frac{p}{3}}+\omega_{\natural}(s, t)\left(\lambda^{-1}+\lambda^{-2}\right)^{\frac{p}{3}}\right) .
\end{aligned}
$$

Choosing $\lambda$ such that $C\left(\lambda^{-1}+\lambda^{-2}\right)^{\frac{p}{3}} \leq \frac{1}{2}$ and $\tilde{L}$ such that $\eta=\omega_{Z}(s, t)^{\frac{1}{p}} \lambda \leq \tilde{L} \lambda \leq 1$, we obtain (4.4).

REMARK 4.2. It is worth noting that we use the decomposition

$$
\delta u_{s \theta t}^{\natural}(\phi)=\left(I-J^{\eta}\right) \delta u_{s \theta t}^{\natural}(\phi)+J^{\eta} \delta u_{s \theta t}^{\natural}(\phi)
$$

and not

$$
\delta u_{s \theta t}^{\natural}(\phi)=\delta u_{s \theta t}^{\natural}\left(\left(I-J^{\eta}\right) \phi\right)+\delta u_{s \theta t}^{\natural}\left(J^{\eta} \phi\right)
$$

in the previous proof in order to avoid using an estimate

$$
u_{s \theta}^{\natural}\left(\mathcal{A}_{\theta t}^{2, *} J^{\eta} \phi\right) \leq \omega_{\natural}(s, t)^{\frac{3}{p}}\left\|\mathcal{A}_{\theta t}^{2, *}\right\|_{5,3}\left\|J^{\eta}\right\|_{2,5}|\phi|_{2},
$$

which, in turn, would require us to impose more regularity on $\sigma$.

We will now prove an a priori estimate of the $p$-variation of the solution $u$.

LEMMA 4.3. Assume that $u$ is a solution of (3.1) up to time $T^{*}$. Then $u \in C^{p \text {-var }}\left(\left[0, T^{*}\right]\right.$; $\left.\mathbf{H}^{0}\right)$ and there is a positive constant $\tilde{L}=\tilde{L}\left(p,|\sigma|_{3, \infty}\right)<L$ such that for all $(s, t) \in \Delta_{T^{*}}$ with $(t-s)+\omega_{Z}(s, t) \leq \tilde{L}$, it holds that

$$
|u|_{p \text {-var; }[s, t] ; \mathbf{H}^{0}}^{p} \lesssim p,|\sigma|_{3, \infty}\left(1+|u|_{L_{T^{*}}^{\infty} \mathbf{H}^{1}}\right)^{2 p}\left((t-s)+\omega_{Z}(s, t)+\omega_{\sharp}(s, t)\right) .
$$

Proof. For all $\eta \in(0,1],(s, t) \in \Delta_{T^{*}}$ and $\phi \in \mathbf{H}^{0}$, we have

$$
\delta u_{s t}(\phi)=\delta u_{s t}\left(J^{\eta} \phi\right)+\delta u_{s t}\left(\left(I-J^{\eta}\right) \phi\right) .
$$

By (2.9), we have

$$
\left|\delta u_{s t}\left(\left(I-J^{\eta}\right) \phi\right)\right| \leq 2|u|_{L_{T^{*}}^{\infty} \mathbf{H}^{1}}\left\|I-J^{\eta}\right\|_{0,-1}|\phi|_{0} \lesssim \eta|u|_{L_{T^{*}}^{\infty} \mathbf{H}^{1}}|\phi|_{0} .
$$

In order to estimate the smooth part, we expand $\delta u_{s t}$ using (4.1) and then apply (2.2) and (2.9) to get

$$
\begin{aligned}
& \left|\delta u_{s t}\left(J^{\eta} \phi\right)\right| \leq\left|\delta \mu_{s t}\left(J^{\eta} \phi\right)\right|+\left|u_{s}\left(\mathcal{A}_{s t}^{1, *} J^{\eta} \phi\right)\right|+\left|u_{s}\left(\mathcal{A}_{s t}^{2, *} J^{\eta} \phi\right)\right|+\left|u_{s t}^{\natural}\left(J^{\eta} \phi\right)\right| \\
& \lesssim\left(\left(1+|u|_{L_{T^{*}}^{\infty} \mathbf{H}^{1}}\right)^{2}(t-s)\left\|J^{\eta}\right\|_{0,1}+|u|_{L_{T^{*}}^{\infty} \mathbf{H}^{1}}\left\|\mathcal{A}_{s t}^{1, *}\right\|_{0,-1}\left\|J^{\eta}\right\|_{0,0}\right. \\
& \left.+|u|_{L_{T^{*}}^{\infty} \mathbf{H}^{1}}\left\|\mathcal{A}_{s t}^{2, *}\right\|_{1,-1}\left\|J^{\eta}\right\|_{0,1}+\omega_{\natural}(s, t)^{\frac{3}{p}}\left\|J^{\eta}\right\|_{0,2}\right)|\phi|_{0} \\
& \lesssim\left(\left(1+|u|_{L_{T^{*}}^{\infty} \mathbf{H}^{1}}\right)^{2}(t-s) \eta^{-1}+|u|_{L_{T^{*}}^{\infty} \mathbf{H}^{1}} \omega_{Z}(s, t)^{\frac{1}{p}}\right.
\end{aligned}
$$

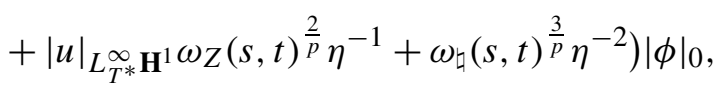


for all $(s, t) \in \Delta_{T^{*}}$ such that $\omega_{Z}(s, t) \leq L$. Setting $\eta=(t-s)^{\frac{1}{p}}+\omega_{Z}(s, t)^{\frac{1}{p}}+\omega_{\natural}(s, t)^{\frac{1}{p}}<1$, we obtain

$$
\left|\delta u_{s t}\right|_{0} \lesssim\left(1+|u|_{L_{T^{*}}^{\infty} \mathbf{H}^{1}}\right)^{2}\left((t-s)+\omega_{Z}(s, t)+\omega_{\natural}(s, t)\right)^{\frac{1}{p}} .
$$

It then follows from [21], Remark 2.3, that $u \in C^{p \text {-var }}\left([0, T] ; \mathbf{H}^{0}\right)$; that is, the local variation spaces agree with the global variation spaces for one-index maps.

The following lemma shows that the solution $u$ is controlled by $\mathcal{A}^{1}$ so that we may construct the rough integral $Q \int_{0}^{\cdot}\left(\sigma_{k} \cdot \nabla+\left(\nabla \sigma_{k}\right)\right) u_{r} d z_{r}^{k}$ needed to recover the pressure.

LEMMA 4.4. Assume that $u$ is a solution of (3.1) up to time $T^{*}$. Then there is a positive constant $\tilde{L}=\tilde{L}\left(p,|\sigma|_{3, \infty}\right)<L$ such that for all $(s, t) \in \Delta_{T^{*}}$ with $\omega_{Z}(s, t) \leq \tilde{L}$, it holds that

$$
\omega_{\sharp}(s, t):=\left|u^{\sharp}\right|_{\frac{p}{2}-\operatorname{var} ;[s, t] ; \mathbf{H}^{-1}}^{\frac{p}{2}} \lesssim_{p,|\sigma|_{3, \infty}}\left(1+|u|_{L_{T^{*}}^{\infty} \mathbf{H}^{1}}\right)^{p}\left((t-s)+\omega_{Z}(s, t)+\omega_{\sharp}(s, t)\right),
$$

where $u^{\sharp}$ is defined by (4.3).

Proof. For all $\eta \in(0,1],(s, t) \in \Delta_{T^{*}}$ and $\phi \in \mathbf{H}^{1}$, we have

$$
u_{s t}^{\sharp}(\phi)=u_{s t}^{\sharp}\left(J^{\eta} \phi\right)+u_{s t}^{\sharp}\left(\left(I-J^{\eta}\right) \phi\right) \text {. }
$$

As explained above, we employ the first formula of (4.3) to estimate the nonsmooth part and the second one to estimate the smooth part. Making use of (2.2) and (2.9), we obtain

$$
\begin{aligned}
\left|u_{s t}^{\sharp}\left(\left(I-J^{\eta}\right) \phi\right)\right| & \leq\left|\delta u_{s t}\left(\left(I-J^{\eta}\right) \phi\right)\right|+\left|u_{s}\left(\mathcal{A}_{s t}^{1, *}\left(I-J^{\eta}\right) \phi\right)\right| \\
& \leq|u|_{L_{T^{*}}^{\infty} \mathbf{H}^{1}}\left(\left\|I-J^{\eta}\right\|_{1,-1}+|u|_{L_{T^{*}}^{\infty} \mathbf{H}^{1}}\left\|\mathcal{A}_{s t}^{1, *}\right\|_{0,-1}\left\|I-J^{\eta}\right\|_{1,0}\right)|\phi|_{1} \\
& \lesssim|u|_{L_{T^{*}}^{\infty} \mathbf{H}^{1}}\left(\eta^{2}+\eta \omega_{Z}(s, t)^{\frac{1}{p}}\right)|\phi|_{1}
\end{aligned}
$$

and

$$
\begin{aligned}
& \left|u_{s t}^{\sharp}\left(J^{\eta} \phi\right)\right| \leq\left|\delta \mu_{s t}\left(J^{\eta} \phi\right)\right|+\left|u_{s}\left(\mathcal{A}_{s t}^{2, *} J^{\eta} \phi\right)\right|+\left|u_{s t}^{\natural}\left(J^{\eta} \phi\right)\right| \\
& \lesssim\left(\left(1+|u|_{L_{T^{*}}^{\infty} \mathbf{H}^{1}}\right)^{2}(t-s)\left\|J^{\eta}\right\|_{1,1}+|u|_{L_{T^{*}}^{\infty} \mathbf{H}^{1}}\left\|\mathcal{A}_{s t}^{2, *}\right\|_{1,-1}\left|J^{\eta}\right|_{1,1}\right. \\
& \left.+\omega_{\natural}(s, t)^{\frac{3}{p}}\left\|J^{\eta}\right\|_{1,2}\right)|\phi|_{1} \\
& \lesssim\left(\left(1+|u|_{L_{T^{*}}^{\infty} \mathbf{H}^{1}}\right)^{2}(t-s)+|u|_{L_{T^{*}}^{\infty} \mathbf{H}^{1}} \omega_{Z}(s, t)^{\frac{2}{p}}+\omega_{\natural}(s, t)^{\frac{3}{p}} \eta^{-1}\right)|\phi|_{1},
\end{aligned}
$$

for all $(s, t) \in \Delta_{T^{*}}$ with $\omega_{Z}(s, t) \leq L$. Setting $\eta=\omega_{Z}(s, t)^{\frac{1}{p}}+\omega_{\natural}(s, t)^{\frac{1}{p}}<1$, we get

$$
\left|u_{s t}^{\sharp}\right|_{-1} \lesssim\left(1+|u|_{L_{T^{*}}^{\infty} \mathbf{H}^{1}}\right)^{2}\left((t-s)^{\frac{p}{2}}+\omega_{Z}(s, t)+\omega_{\sharp}(s, t)\right)^{\frac{2}{p}},
$$

which proves the claim.

Finally, we have all in hand to show how to recover the pressure in the original equation (4.1). The computation in the proof shows why (3.15) is the correct Chen's relation for this system.

PROOF OF LEMMA 3.8. We first show that we can construct the following rough integral using the sewing lemma, [21], Lemma B.1:

$$
I_{t}=Q \int_{0}^{t}\left[\sigma_{k} \cdot \nabla+\left(\nabla \sigma_{k}\right)\right] u_{r} d z_{r}^{k}, \quad I_{0}=0 .
$$


Let $h_{s t}=\mathcal{A}_{s t}^{Q, 1} u_{s}+\mathcal{A}_{s t}^{Q, 2} u_{s}$ for $(s, t) \in \Delta_{T^{*}}$. Owing to (2.2), we have $\left|h_{s t}\right|_{-1} \leq\left\|\mathcal{A}_{s t}^{Q, 1, *}\right\|_{0,-1}|u|_{L_{T^{*}}^{\infty} \mathbf{H}^{1}}+\left\|\mathcal{A}_{s t}^{Q, 2, *}\right\|_{1,-1}|u|_{L_{T^{*}}^{\infty} \mathbf{H}^{1}} \leq|u|_{L_{T^{*}}^{\infty} \mathbf{H}^{1}} \omega_{Z}(s, t)^{\frac{1}{p}}+\omega_{Z}(s, t)^{\frac{2}{p}}$, which implies that $h \in C_{2}^{p \text {-var }}\left([0, T] ; \mathbf{H}_{\perp}^{-1}\right)$. Applying (3.15), we find $\delta h_{s \theta t}=-\mathcal{A}_{\theta t}^{Q, 1} u_{s \theta}^{\sharp}-$ $\mathcal{A}_{\theta t}^{Q, 2} \delta u_{s \theta}$. By virtue of Lemmas 4.3 and 4.4 , there are controls $\omega$ and $\varpi(s, t)=(t-s)+$ $\omega_{Z}(s, t)$ and a positive constant $\tilde{L}<L$ such that for all $(s, \theta, t) \in \Delta_{T^{*}}^{(2)}$ with $\varpi(s, t) \leq \tilde{L}$, we have

$$
\begin{aligned}
\left|\delta h_{s \theta t}\right|_{-2} & \leq\left\|\mathcal{A}_{\theta t}^{Q, 1, *}\right\|_{2,1}\left|u_{s \theta}^{\sharp}\right|_{-1}+\left\|\mathcal{A}_{\theta t}^{Q, 2, *}\right\|_{2,0}\left|\delta u_{s \theta}\right|_{0} \\
& \lesssim\left(\omega_{Z}(s, t)^{\frac{1}{3}} \omega_{\sharp}(s, t)^{\frac{2}{3}}+\omega_{Z}(s, t)^{\frac{2}{3}} \omega_{u}(s, t)^{\frac{1}{3}}\right)^{\frac{3}{p}}=: \omega(s, t)^{\frac{3}{p}} .
\end{aligned}
$$

Therefore, by [21], Lemma B.1 and Remark 2.3, there is a unique path $I \in C^{p \text {-var }}([0, T]$; $\left.\mathbf{H}_{\perp}^{-2}\right)$ and a two-index map $I^{\natural} \in C_{2, \varpi, \tilde{L}}^{p \text {-var }}\left([0, T] ; \mathbf{H}_{\perp}^{-2}\right)$ such that

$$
\delta I_{s t}=\mathcal{A}_{s t}^{Q, 1} u_{s}+\mathcal{A}_{s t}^{Q, 2} u_{s}+I_{s t}^{\natural},
$$

and $\left|I_{s t}^{\natural}\right|_{-2} \lesssim \omega(s, t)^{\frac{3}{p}}$ for all $(s, t) \in \Delta_{T^{*}}$ with $\varpi(s, t) \leq \tilde{L}$. Defining $\pi_{t}:=-\int_{0}^{t} B_{Q}\left(u_{r}\right) d r+$ $I_{t}$, yields (3.14) with $u^{Q, \natural}:=I^{\natural}$. Applying (2.4), we find that $\pi \in C^{p-\operatorname{var}}\left([0, T] ; \mathbf{H}_{\perp}^{-2}\right)$, which completes the proof.

When proving existence using a Galerkin approximation, we will use Definition 3.5 to find estimates as indicated by Theorem 3.10, since $\xi$ satisfies an enstrophy balance. However, using the Biot-Savart operator only yields an estimate on the mean-free part of the velocity $v=u-\bar{u}$. The next lemma shows how to bound the mean, $\bar{u}$, in terms of $v$.

LEMMA 4.5. Assume $(\xi, \bar{u})$ is a solution of (3.8) up to time $T^{*}$. Then there is a positive constant $C=C\left(p,|\sigma|_{3, \infty}, T^{*}, \omega_{Z}\right)$ such that

$$
|\bar{u}|_{L_{T^{*}}^{\infty} \mathbf{R}^{d}} \leq C \exp \left\{C\left(1+|v|_{L_{T^{*}}^{\infty} \mathbf{H}^{1}}\right)^{p}\right\}\left(1+\left|\bar{u}_{0}\right|\right) .
$$

PROOF. Making use of (3.12), the reverse triangle inequality, and (3.11), we find that for all $(s, t) \in \Delta_{T^{*}}$,

$$
\delta(|\bar{u}|)_{s t} \leq\left|\bar{u}_{s t}^{\natural}\right|+\left|\left[L_{s t}^{1}+L_{s t}^{2}\right]\left(v_{s}\right)\right| \lesssim\left|\bar{u}_{s t}^{\natural}\right|+|v|_{L_{T^{*}}^{\infty} \mathbf{H}^{0}}\left(\omega_{Z}(s, t)^{\frac{1}{p}}+\omega_{Z}(s, t)^{\frac{2}{p}}\right) .
$$

By Lemma 3.6, we have $\left|\bar{u}^{\natural}\right| \leq\left|u^{\natural}\right|-2$. Inspecting the proof of Lemma 4.1, we deduce that for all $(s, t) \in \Delta_{T^{*}} \omega_{Z}(s, t) \leq \tilde{L}$,

$$
\left|u_{s t}^{\natural}\right|_{-2} \lesssim|u|_{L^{\infty}\left([s, t] ; \mathbf{H}^{1}\right)} \omega_{Z}(s, t)^{\frac{3}{p}}+\frac{\left|\delta \mu_{s t}\right|_{-1}}{t-s}(t-s) \omega_{Z}(s, t)^{\frac{1}{p}} .
$$

Writing $u=\bar{u}+v$, by the bilinearity of $B_{P}$ and the fact that $\nabla u=\nabla v$, we obtain

$$
\left|B_{P}(u, u)\right|_{-1}=\left|B_{P}(u, v)\right|_{-1} \leq\left|B_{P}(\bar{u}, v)\right|_{-1}+\left|B_{P}(v, v)\right|_{-1} \lesssim|\bar{u}||v|_{1}+|v|_{1}^{2},
$$

where the last inequality follows from setting $m_{1}=m_{3}=1$ and $m_{2}=0$ in (2.4). Thus,

$$
\frac{\left|\delta \mu_{s t}\right|_{-1}}{t-s} \lesssim|\bar{u}|_{L^{\infty}\left([s, t] ; \mathbf{R}^{d}\right)}|v|_{L_{T^{*}}^{\infty} \mathbf{H}^{1}}+|v|_{L_{T^{*}}^{\infty} \mathbf{H}^{1}}^{2},
$$


which is a more refined bound than (4.2) that is linear in $|\bar{u}|_{L^{\infty}([s, t])}$. Combining the above bounds, we get that there is a constant $C=C\left(p,|\sigma|_{3, \infty}, \tilde{L}, T^{*}\right)>0$ such that for all $(s, t) \in$ $\Delta_{T *}$ with $\omega_{Z}(s, t) \leq \tilde{L}$,

$$
\begin{aligned}
\delta(|\bar{u}|)_{s t} \lesssim & |\bar{u}|_{L^{\infty}\left([s, t] ; \mathbf{R}^{d}\right)}\left(\omega_{Z}(s, t)^{\frac{2}{p}}+|v|_{L_{T^{*}}^{\infty} \mathbf{H}^{1}}(t-s)\right) \omega_{Z}(s, t)^{\frac{1}{p}} \\
& +\left(1+|v|_{L_{T^{*}}^{\infty} \mathbf{H}^{1}}\right)^{2}\left(\omega_{Z}(s, t)^{\frac{1}{p}}+\omega_{Z}(s, t)^{\frac{2}{p}}+\omega_{Z}(s, t)^{\frac{3}{p}}+(t-s) \omega_{Z}(s, t)^{\frac{1}{p}}\right) \\
\leq & C\left(|\bar{u}|_{L^{\infty}\left([s, t] ; \mathbf{R}^{d}\right)} \omega_{Z}(s, t)^{\frac{1}{p}}+\left(1+|v|_{L_{T^{*}}^{\infty} \mathbf{H}^{1}}\right)^{2} \omega_{Z}(s, t)^{\frac{1}{p}}\right) .
\end{aligned}
$$

Applying Lemma A.2 with $\omega=C\left(1+|v|_{L_{T^{*}}^{\infty} \mathbf{H}^{1}}\right)^{p} \omega_{Z}$ and $\phi=C\left(1+|v|_{L_{T^{*}}^{\infty} \mathbf{H}^{1}}\right)^{2} \omega_{Z}^{\frac{1}{p}}$, we find

$$
|\bar{u}|_{L_{T^{*}}^{\infty} \mathbf{R}^{d}} \leq 2 \exp \left\{C\left(1+|v|_{L_{T^{*}}^{\infty} \mathbf{H}^{1}}\right)^{p} \omega_{Z}(0, T *)\right\}\left(\left|\bar{u}_{0}\right|+K\left(1+|v|_{L_{T^{*}}^{\infty} \mathbf{H}^{1}}\right)^{2} \omega_{Z}(0, T *)^{\frac{1}{p}}\right) .
$$

5. Enstrophy balance and uniqueness in two spatial dimensions. This section is devoted to the proof of Theorem 3.10 and Corollary 3.12, which we split into three parts. First, we establish the enstrophy balance (3.16) in Section 5.1. Second, we prove uniqueness in Section 5.2. Third, we show stability in Section 5.3.

Throughout this section, we let $d=2$. In particular, the vorticity $\xi$ is scalar valued and consequently the associated function spaces contain functions that are scalar valued. Since the dimension will always be clear from the context, we do not alter the notation introduced in Section 2.1.

5.1. Enstrophy balance. In the classical setting, to show (3.16), one would test (3.8) by the solution $\xi$ and use that $u$ and $\sigma_{k}$ are divergence-free. Since $\xi^{\natural}$ is not expected to be better behaved than a spatial distribution, one cannot directly test the equation by the solution itself. Instead, we employ a standard trick in PDE theory, namely, the doubling of variables technique. Define the tensor $\xi \otimes \zeta(x, y):=\xi(x) \zeta(y)$, the symmetric tensor $\xi \hat{\otimes} \zeta=$ $\frac{1}{2}(\xi \otimes \zeta+\zeta \otimes \xi)$, and the scale of Sobolev spaces $W_{\otimes}^{n, 2}:=W^{n, 2}\left(\mathbf{T}^{2} \times \mathbf{T}^{2}\right)$.

Variations of the following result have already been proved in $[12,18,19,21]$, so we omit the proof.

Proposition 5.1. The mapping $\xi^{\otimes 2}:[0, T] \rightarrow W_{\otimes}^{0,2}$ satisfies the equation

$$
\delta \xi_{s t}^{\otimes 2}=\int_{s}^{t} 2\left[\vartheta \xi_{r} \hat{\otimes} \Delta \xi_{r}-\xi_{r} \hat{\otimes} u_{r} \nabla \xi_{r}\right] d r+\left(\Gamma_{s t}^{1}+\Gamma_{s t}^{2}\right) \xi_{s}^{\otimes 2}+\xi_{s t}^{\otimes 2, \natural}
$$

in $W_{\otimes}^{-3,2}$. Here, $\left(\Gamma^{1}, \Gamma^{2}\right)$ is the unbounded rough driver on $\left(W_{\otimes}^{n, 2}\right)_{n}$ defined by the second quantization

$$
\Gamma^{1}:=2 A^{1} \hat{\otimes} I, \quad \Gamma^{2}:=A^{2} \hat{\otimes} I+A^{1} \otimes A^{1},
$$

and $\xi^{\otimes 2, \natural} \in C_{2, \omega_{Z}, L}^{\frac{p}{3}-v a r}\left([0, T] ; W_{\otimes}^{-3,2}\right)$ for some $L>0$.

The next step is to test $\xi^{\otimes 2}$ against an approximation of $\delta_{x=y}$ so that we can justify the testing of $\xi$ against itself; that is, to justify the evaluation of $\xi \otimes \xi$ at the diagonal $x=y$. As usual, in the framework of unbounded rough drivers, in order to obtain estimates, we shall rewrite the approximation in the standard form (2.10) and use Theorem 2.6. The two ingredients in Theorem 2.6-the scale of spaces and the corresponding family of smoothing operators-will be constructed in this section. 
The approximation of $\delta_{x=y}$ we shall use will increase the support of the solution, which could pose a problem when working, for instance, on a bounded domain other than $\mathbf{T}^{2}$. Here, we recall that we are blurring the distinction between $2 \pi$-periodic functions defined on the whole space and functions defined on the torus $\mathbf{T}^{2}$. However, for $f \in L^{1}\left(\mathbf{T}^{2}\right)$, we have

$$
\int_{B(0,1)+\mathbf{T}^{2}}|f(x)| d x \leq \int_{2 \mathbf{T}^{2}}|f(x)| d x=2 \int_{\mathbf{T}^{2}}|f(x)| d x,
$$

which means that the increase of the support of integration is always a continuous operation on $L^{1}\left(\mathbf{T}^{2}\right)$.

Introduce the coordinates $x_{+}:=\frac{x+y}{2}$ and $x_{-}:=\frac{x-y}{2}$ and denote by $\nabla_{ \pm}:=\nabla_{x} \pm \nabla_{y}$. We consider test functions that are periodic in the $x_{+}$direction and compactly supported in the $x_{-}$direction. More precisely, for $n \in \mathbf{N}$, we define the Banach spaces

$$
\begin{aligned}
\mathcal{E}_{\nabla}^{n}:= & \left\{\Phi \in W^{n, \infty}\left(\mathbf{R}^{2} \times \mathbf{R}^{2}\right): \Phi(x+k 2 \pi, y+k 2 \pi)=\Phi(x, y),\right. \\
& \left.\forall k \in \mathbf{Z}^{2} \text { and }\left|x_{-}\right| \geq 1 \Rightarrow \Phi(x, y)=0\right\}
\end{aligned}
$$

equipped with the norm

$$
|\Phi|_{n, \nabla}:=\max _{k+l \leq n} \operatorname{esssup}\left\{\left|\nabla_{+}^{k} \nabla_{-}^{l} \Phi\left(x_{+}+x_{-}, x_{+}-x_{-}\right)\right|: x_{+} \in \mathbf{T}^{2}, x_{-} \in \mathbf{R}^{2}\right\} .
$$

We then define $\left.\mathcal{E}_{\nabla}^{n}=\left(\mathcal{E}_{\nabla}^{-n}\right)\right)^{*}$ for $n<0$. We also define the duality pairing between $\mathcal{E}_{\nabla}^{-n}$ and $\mathcal{E}_{\nabla}^{n}$ by

$$
\langle\Phi, \Psi\rangle_{\nabla}=\int_{\mathbf{T}^{2}} \int_{\mathbf{R}^{2}} \Phi\left(x_{+}+x_{-}, x_{+}-x_{-}\right) \Psi\left(x_{+}+x_{-}, x_{+}-x_{-}\right) d x_{-} d x_{+},
$$

with the obvious abuse of notation. Notice that the test functions in (5.2) are not periodic in the original variables $x, y$ separately, only in $x_{+}$. In addition, due to the compact support of the test functions in the $x_{-}$variable, the domain of integration in the duality product above can be written in the $(x, y)$-coordinates and is equal to

$$
\Omega=\left\{(x, y): x_{+} \in \mathbf{T}^{2}, x_{-} \in B(0,1)\right\} .
$$

Define the blow-up transformation for $\epsilon \in(0,1)$ by

$$
T_{\epsilon} \Phi(x, y)=\epsilon^{-2} \Phi\left(x_{+}+\frac{x_{-}}{\epsilon}, x_{+}-\frac{x_{-}}{\epsilon}\right) .
$$

The dual of $T_{\epsilon}$ with respect to $\langle\cdot, \cdot\rangle_{\nabla}$ is given by

$$
T_{\epsilon}^{*} \Phi(x, y)=\Phi\left(x_{+}+\epsilon x_{-}, x_{+}-\epsilon x_{-}\right)
$$

and $T_{\epsilon}^{-1}=\epsilon^{2} T_{\epsilon}^{*}$.

We shall require the following uniform estimates.

LEMMA 5.2. For all $f \in L^{2}$ and $g, h \in W^{1,2}$,

$$
\left|T_{\epsilon}^{*}\left(f^{\otimes 2}\right)\right|_{-0, \nabla} \lesssim|f|_{L^{2}\left(\mathbf{T}^{2}\right)}^{2} \quad \text { and } \quad\left|T_{\epsilon}^{*}(g \otimes \Delta h)\right|_{-1, \nabla} \lesssim|g|_{1}|h|_{1} .
$$

Proof. By Hölder's inequality, for all $f \in L^{2}$ we find

$$
\begin{aligned}
\left|\left\langle T_{\epsilon}^{*} f^{\otimes 2}, \Phi\right\rangle_{\nabla}\right| & =\left|\int_{\mathbf{R}^{2}} \int_{\mathbf{T}^{2}} f\left(x_{+}+\epsilon x_{-}\right) f\left(x_{+}-\epsilon x_{-}\right) \Phi\left(x_{+}+x_{-}, x_{+}-x_{-}\right) d x_{+} d x_{-}\right| \\
& \leq \max _{\tau \in\{-1,1\}} \int_{B(0,1)} \int_{\mathbf{T}^{2}}\left|f\left(x_{+}+\tau \epsilon x_{-}\right)\right|^{2} d x_{+} \sup _{x_{+}}\left|\Phi\left(x_{+}+x_{-}, x_{+}-x_{-}\right)\right| d x_{-} .
\end{aligned}
$$


Thus, making the change of variables $z_{\tau}=x_{+}+\tau \epsilon x_{-} \in \mathbf{T}^{2} \pm \epsilon B(0,1) \subset \mathbf{T}^{2}+B(0,1)$ we obtain

$$
\left|\left\langle T_{\epsilon}^{*} f^{\otimes 2}, \Phi\right\rangle_{\nabla}\right| \leq|B(0,1)||\Phi|_{0, \nabla} \int_{\mathbf{T}^{2}+B(0,1)}|f(x)|^{2} d x \lesssim|\Phi|_{0, \nabla}|f|_{L^{2}\left(\mathbf{T}^{2}\right)}^{2},
$$

which proves the first estimate in (5.3).

For all $g, h \in W^{1,2}$, we have

$$
g \otimes \Delta h=\nabla_{y} \cdot(g \otimes \nabla h)=\left(\nabla_{+}-\nabla_{x}\right) \cdot(g \otimes \nabla h)=\nabla_{+} \cdot(g \otimes \nabla h)-\nabla g \otimes \nabla h .
$$

A simple computation shows $\nabla_{+} T_{\epsilon}=T_{\epsilon} \nabla_{+}$. Thus, using that the dual of $\nabla_{+}$is $-\nabla_{+}$, we find

$$
\begin{aligned}
\left\langle T_{\epsilon}^{*}(g \otimes \Delta h), \Phi\right\rangle_{\nabla} & \\
= & -\left\langle T_{\epsilon}^{*}(g \otimes \nabla h), \nabla_{+} \Phi\right\rangle_{\nabla}-\left\langle T_{\epsilon}^{*}(\nabla g \otimes \nabla h), \Phi\right\rangle_{\nabla} \\
= & -\int_{\mathbf{R}^{2}} \int_{\mathbf{T}^{2}} g\left(x_{+}+\epsilon x_{-}\right) \nabla h\left(x_{+}-\epsilon x_{-}\right) \nabla_{+} \Phi\left(x_{+}+x_{-}, x_{+}-x_{-}\right) d x_{+} d x_{-} \\
& -\int_{\mathbf{R}^{2}} \int_{\mathbf{T}^{2}} \nabla g\left(x_{+}+\epsilon x_{-}\right) \nabla h\left(x_{+}-\epsilon x_{-}\right) \Phi\left(x_{+}+x_{-}, x_{+}-x_{-}\right) d x_{+} d x_{-} .
\end{aligned}
$$

Following a similar derivation of the estimate $\left\langle T_{\epsilon}^{*} f^{\otimes 2}, \Phi\right\rangle_{\nabla}$, we get

$$
\begin{aligned}
\left|\left\langle T_{\epsilon}^{*}(g \otimes \Delta h), \Phi\right\rangle_{\nabla}\right| \lesssim & |g|_{L^{2}\left(\mathbf{T}^{2}+B(0,1)\right)}|\nabla h|_{L^{2}\left(\mathbf{T}^{2}+B(0,1)\right)}|\Phi|_{1, \nabla} \\
& +|\nabla g|_{L^{2}\left(\mathbf{T}^{2}+B(0,1)\right)}|\nabla h|_{L^{2}\left(\mathbf{T}^{2}+B(0,1)\right)}|\Phi|_{0, \nabla} \\
\lesssim & |g|_{1}|h|_{1}|\Phi|_{1, \nabla},
\end{aligned}
$$

which proves the second estimate in (5.3).

In order to be able to apply Theorem 2.6, we need to construct a family of smoothing operators on the scale $\left(\mathcal{E}_{\nabla}^{n}\right)_{n}$. Recall that $\Omega \subset\left(2 \mathbf{T}^{2}\right) \times\left(2 \mathbf{T}^{2}\right)$. We may therefore choose a mollifier (in both variables) $\left\{\rho_{\eta}\right\}_{\eta \in(0,1]}$ such that $\operatorname{supp}\left(\rho_{\eta}\right) \subset B(0, \eta) \subset \Omega$ and for all $\Phi \in \mathcal{E}_{\nabla}^{n}$ we have

$$
\begin{aligned}
J^{\eta} \Phi(x, y) & :=\int_{\Omega} \Phi(x+\tilde{x}, y+\tilde{y}) \rho_{\eta}(\tilde{x}, \tilde{y}) d \tilde{x} d \tilde{y} \\
& =\int_{\left(2 \mathbf{T}^{2}\right) \times\left(2 \mathbf{T}^{2}\right)} \Phi(x+\tilde{x}, y+\tilde{y}) \rho_{\eta}(\tilde{x}, \tilde{y}) d \tilde{x} d \tilde{y} .
\end{aligned}
$$

It follows that $J^{\eta}$ acts as a smoothing operator on the scale $W^{n, \infty}\left(\left(2 \mathbf{T}^{2}\right) \times\left(2 \mathbf{T}^{2}\right)\right)$. We could try to restrict to $\mathcal{E}_{\nabla}^{n}$, but the problem is that our test function space is constructed such that $\Phi(x, y)=0$ when $\left|x_{-}\right| \geq 1$, and convolution increases this support. However, the increase cannot be too large because

$$
\operatorname{supp}_{x_{-}}\left(J^{\eta} \Phi\right) \subset \operatorname{supp}_{x_{-}}(\Phi)+\operatorname{supp}\left(\rho_{\eta}\right) \subset B(0,1)+B(0, \eta) \subset B(0,1+\eta),
$$

where $\operatorname{supp}_{x_{-}}$denotes the projection on the support on the $x_{-}$direction. In other words, our smoothing operator is not well defined as a mapping from $\mathcal{E}_{\nabla}^{n}$ into itself. We work around this by introducing a function that decreases the support by $\eta$.

LEMMA 5.3. There exists a family of smoothing operators $\left(\bar{J}^{\eta}\right)_{\eta \in[0,1]}$ on the scale $\left(\mathcal{E}_{\nabla}^{n}\right)_{n}$. 
Proof. Let $\theta_{\eta}: \mathbf{R} \rightarrow[0,1]$ be a smooth function such that

$$
\theta_{\eta}(\zeta)= \begin{cases}1 & \text { if }|\zeta| \leq 1-3 \eta \\ 0 & \text { if }|\zeta| \geq 1-2 \eta\end{cases}
$$

and $\left|\nabla^{k} \theta_{\eta}\right|_{\infty} \lesssim \eta^{-k}$ for $k \in\{1,2\}$. Define $\Theta_{\eta}(x, y)=\theta_{\eta}\left(x_{-}\right)$. In [12], Proposition 5.3, it was shown that

$$
\left|\left(I-\Theta_{\eta}\right) \Phi\right|_{0, \nabla} \lesssim \eta^{k}|\Phi|_{k, \nabla}, \quad\left|\Theta_{\eta} \Phi\right|_{k, \nabla} \lesssim|\Phi|_{k, \nabla}
$$

for $k \in\{0,1,2\}$. Moreover, for every $\Phi \in \mathcal{E}_{\nabla}^{n}$, we have

$$
\operatorname{supp}_{x_{-}}\left(J^{\eta} \Theta_{\eta} \Phi\right) \subset \operatorname{supp}_{x_{-}}\left(\Theta_{\eta} \Phi\right)+B(0, \eta) \subset B(0,1-\eta)+B(0, \eta) \subset B(0,1),
$$

so that $\bar{J}^{\eta}:=J^{\eta} \Theta_{\eta}$ is a well defined operator on $\mathcal{E}_{\nabla}^{n}$. Similarly, we have $\operatorname{supp}_{x_{-}}((I-$ $\left.\left.J^{\eta}\right) \Phi\right) \subset \operatorname{supp}_{x_{-}}(\Phi)+\operatorname{supp}\left(\rho_{\eta}\right)$ and $\operatorname{supp}_{x_{-}}\left(\Theta_{\eta} \Phi\right) \subset B(0,1-2 \eta)$ we have that $(I-$ $\left.J^{\eta}\right) \Theta_{\eta} \Phi \in \mathcal{E}_{\nabla}^{n}$ for every $\Phi \in \mathcal{E}_{\nabla}^{n}$.

It remains to show (2.9). The second estimate is obvious. The first follows from the equality

$$
\left(I-\bar{J}^{\eta}\right) \Phi=\left(I-J^{\eta}\right) \Phi+J^{\eta}\left(I-\Theta_{\eta}\right) \Phi,
$$

together with the estimates in (5.4).

We are now ready to derive the equation for $\xi^{2}$. To do this, we evaluate (5.1) in $T_{\epsilon} \Phi$ for any $\Phi \in \mathcal{E}_{\nabla}^{3}$ to get

$$
\delta \xi_{s t}^{\epsilon, 2}=2 \int_{s}^{t}\left[\vartheta T_{\epsilon}^{*}\left(\xi_{r} \hat{\otimes} \Delta \xi_{r}\right)-T_{\epsilon}^{*}\left(\xi_{r} \hat{\otimes} u_{r} \cdot \nabla \xi_{r}\right)\right] d r+\left(\Gamma_{s t}^{1, \epsilon}+\Gamma_{s t}^{2, \epsilon}\right) \xi_{s}^{\epsilon, 2}+\xi_{s t}^{\epsilon, 2, \sharp}
$$

where we have defined

$$
\xi^{\epsilon, 2}:=T_{\epsilon}^{*} \xi^{\otimes 2}, \quad \Gamma_{s t}^{i, \epsilon}:=T_{\epsilon}^{*} \Gamma^{i} T_{\epsilon}^{*,-1}, \quad \xi^{\epsilon, 2, \natural}:=T_{\epsilon}^{*} \xi^{\otimes 2, \natural} .
$$

Our goal now is to take the limit as $\epsilon \rightarrow 0$. To this end, we shall derive uniform in $\epsilon$ bounds on the unbounded rough driver $\left(\Gamma^{1, \epsilon}, \Gamma^{2, \epsilon}\right)$ as well as the drift. Then we apply Theorem 2.6 to bound the remainder $\xi^{\epsilon, 2, \natural}$ in terms of the drift and the unbounded rough driver. Notice that this is possible since the equation is satisfied on the scale $\mathcal{E}_{\nabla}^{n}$ and we have defined a smoothing operator on this scale.

The first task is to show that the unbounded rough driver and the drift are uniformly bounded in $\epsilon$. For the proof that the unbounded rough driver is uniformly bounded in $\epsilon$, we refer to $[12,18]$ and $[2]$ and state the desired result in Proposition 5.4 below. The uniform bound on the drift will be formulated in Lemma 5.5 below.

Proposition 5.4. Assume that $\sigma \in\left(\mathbf{W}^{3, \infty}\right)^{K}$ and $\mathbf{Z} \in \mathcal{C}_{g}^{p \text {-var }}\left([0, T] ; \mathbf{R}^{K}\right)$. Then $\left(\Gamma^{1, \epsilon}, \Gamma^{2, \epsilon}\right)_{\epsilon}$ is a bounded family of unbounded rough drivers on $\mathcal{E}_{\nabla}^{n}$. Moreover, for $\Phi(x, y)=\psi\left(2 x_{-}\right) \phi\left(x_{+}\right)$, where $\psi$ is nonnegative, smooth, has compact support in $(-1,1)$ and $\int_{\mathbf{R}} \psi=1$, and $\phi \in W^{3, \infty}$, we have

$$
\lim _{\epsilon \downarrow 0}\left(\xi_{s}^{\epsilon, 2}, \Gamma_{s t}^{i, \epsilon, *} \Phi\right)_{\nabla}=\left(\xi^{2}, A_{s t}^{i, *} \phi\right), \quad i \in\{1,2\} .
$$

We now show that the drift is uniformly bounded in $\epsilon$. This allows us to take the limit as $\epsilon \rightarrow 0$ in the approximation of $\delta_{x=y}$. 
LEMMA 5.5. There exists a control $\omega$ such that

$$
\left|\int_{s}^{t}\left[\vartheta T_{\epsilon}^{*}\left(\xi_{r} \hat{\otimes} \Delta \xi_{r}\right)-T_{\epsilon}^{*}\left(\xi_{r} \hat{\otimes} u_{r} \cdot \nabla \xi_{r}\right)\right] d r\right|_{-1, \nabla} \leq \omega(s, t) .
$$

Moreover, for $\Phi(x, y)=\psi\left(2 x_{-}\right) \phi\left(x_{+}\right)$where $\psi$ is nonnegative, smooth, has compact support in $(-1,1)$ and $\int_{\mathbb{R}} \psi=1$, and $\phi \in W^{3, \infty}$, we have

$$
\begin{aligned}
\lim _{\epsilon \downarrow 0} \int_{s}^{t}\left\langle\vartheta T_{\epsilon}^{*}\left(\xi_{r} \hat{\otimes} \Delta \xi_{r}\right)-T_{\epsilon}^{*}\left(\xi_{r} \hat{\otimes} u_{r} \cdot \nabla \xi_{r}\right), \Phi\right\rangle_{\nabla} d r \\
\quad=\int_{s}^{t}\left(\vartheta\left(\xi_{r}^{2}, \Delta \phi\right)-2 \vartheta\left(\left|\nabla \xi_{r}\right|^{2}, \phi\right)+\left(\xi_{r}^{2}, \nabla \cdot\left(u_{r} \phi\right)\right)\right) d r .
\end{aligned}
$$

PROOF. By Lemma 5.2, we have

$$
\left|\int_{s}^{t}\left\langle T_{\epsilon}^{*}\left(\xi_{r} \hat{\otimes} \Delta \xi_{r}\right), \Phi\right\rangle_{\nabla} d r\right| \lesssim \int_{s}^{t}\left|\nabla \xi_{r}\right|_{L^{2}\left(\mathbf{T}^{2}+B(0,1)\right)}^{2} d r|\Phi|_{1, \nabla} \lesssim \int_{s}^{t}\left|\nabla \xi_{r}\right|_{0}^{2} d r|\Phi|_{1, \nabla},
$$

and $\int_{s}^{t}\left|\nabla \xi_{r}\right|_{0}^{2} d r$ may be regarded as a control. It follows that

$$
\begin{aligned}
& \mid\left\langle T_{\epsilon}^{*}\left(\xi_{r} \hat{\otimes} u_{r} \cdot \nabla \xi_{r}\right),\left.\Phi\right|_{\nabla}\right| \\
& \quad \leq|\Phi|_{0, \nabla} \int_{B(0,1)} \int_{\mathbf{T}^{2}}\left|\xi_{r}\left(x_{+}+\epsilon x_{-}\right) u_{r}\left(x_{+}-\epsilon x_{-}\right) \cdot \nabla \xi_{r}\left(x_{+}-\epsilon x_{-}\right)\right| d x_{+} d x_{-} \\
& \quad \leq|\Phi|_{0, \nabla}\left|\xi_{r}\right|_{L^{4}\left(\mathbf{T}^{2}+B(0,1)\right)}\left|u_{r}\right|_{L^{4}\left(\mathbf{T}^{2}+B(0,1)\right)}\left|\nabla \xi_{r}\right|_{L^{2}\left(\mathbf{T}^{2}+B(0,1)\right)} \lesssim|\Phi|_{0, \nabla}\left|\xi_{r}\right|_{1}^{2}\left|u_{r}\right|_{1},
\end{aligned}
$$

where we have used the two-dimensional version of Ladyzhenskaya's inequality $|\phi|_{L^{4}} \lesssim|\phi|_{1}$ and the inequality $|\phi|_{L^{p}\left(\mathbf{T}^{2}+B(0,1)\right)} \lesssim|\phi|_{L^{p}\left(\mathbf{T}^{2}\right)}$. Thus,

$$
\left|\int_{s}^{t} T_{\epsilon}^{*}\left(\xi_{r} \hat{\otimes} u_{r} \cdot \nabla \xi_{r}\right) d r\right|_{-0, \nabla} \lesssim \int_{s}^{t}\left|\xi_{r}\right|_{1}^{2}\left|u_{r}\right|_{1} d r \leq|u|_{L_{T}^{\infty} \mathbf{H}^{1}} \int_{s}^{t}\left|\xi_{r}\right|_{1}^{2} d r
$$

and the right-hand-side may be regarded as a control. This shows the first part of the statement.

The second part follows by noticing that for $\Phi(x, y)=\psi\left(2 x_{-}\right) \phi\left(x_{+}\right)$we have $T_{\epsilon} \Phi(x$, $y)=\psi_{\epsilon}\left(2 x_{-}\right) \phi\left(x_{+}\right)$where $\psi_{\epsilon}$ converges to a Dirac-delta. In particular, standard arguments show that

$$
\lim _{\epsilon \downarrow 0}\left\langle\xi_{r} \otimes \Delta \xi_{r}, \psi_{\epsilon} \phi\right\rangle_{\nabla}=-\left(\left|\nabla \xi_{r}\right|_{0}^{2}, \phi\right)-\left(\left(\xi_{r} \cdot \nabla\right) \xi_{r}, \nabla \phi\right)
$$

and

$$
\lim _{\epsilon \downarrow 0}\left|\xi_{r} \otimes\left(u_{r} \cdot \nabla\right) \xi_{r}, \psi_{\epsilon} \phi\right\rangle_{\nabla}=\left(\left(u_{r} \cdot \nabla\right) \xi_{r}, \xi_{r} \phi\right),
$$

for all $r$ such that $\xi_{r} \in W^{1,2}$.

We are now ready to derive the equation for $\xi^{2}$.

THEOREM 5.6. Assume that $\sigma \in\left(\mathbf{W}_{\mathrm{div}}^{3, \infty}\right)^{K}$ and $\mathbf{Z} \in \mathcal{C}_{g}^{p \text {-var }}\left([0, T] ; \mathbf{R}^{K}\right)$. Then $\xi^{2}$ satisfies for every $\phi \in W^{3, \infty}$,

$$
\begin{aligned}
\delta \xi_{s t}^{2}(\phi)= & -\int_{s}^{t}\left[2 \vartheta\left(\left|\nabla \xi_{r}\right|^{2}, \phi\right)-\vartheta\left(\xi_{r}^{2}, \Delta \phi\right)-2\left(\xi_{r}^{2}, \nabla \cdot\left(u_{r} \phi\right)\right)\right] d r \\
& +\left(\xi_{s}^{2},\left[A_{s t}^{1, *}+A_{s t}^{2, *}\right] \phi\right)+\xi_{s t}^{2, \natural}(\phi),
\end{aligned}
$$

where $\xi^{2, \natural} \in C_{2, \omega_{Z}, \tilde{L}}^{\frac{p}{3}-v a r}\left([0, T] ; W^{-3, \infty}\right)$ for some positive constant $\tilde{L}=\tilde{L}\left(p,|\sigma|_{3, \infty}\right)<L$. In particular, $\xi \in C_{T} \mathbf{H}^{0}$ and the enstrophy balance (3.16) holds. 
PROOF. By Proposition 5.4, Lemma 5.5 and Theorem 2.6, there are controls $\omega_{2, \natural}$ and a positive constant $\tilde{L}=\tilde{L}\left(p,|\sigma|_{3, \infty}\right)<L$ independent of $\epsilon>0$ such that $\left|\xi_{s t}^{\epsilon, 2, \natural}\right|_{-3, \nabla} \leq$ $\omega_{2, \sharp}(s, t)^{\frac{3}{p}}$ for all $(s, t) \in \Delta_{T}$ with $\omega_{Z}(s, t) \leq \tilde{L}$. Testing (5.5) against $\Phi(x, y)=$ $\psi\left(2 x_{-}\right) \phi\left(x_{+}\right)$as in Lemma 5.5 and letting $\epsilon \rightarrow 0$, we get (5.6). Indeed, in view of Proposition 5.4 and Lemma 5.5 the drift and rough driver terms converge. For the remainder, we observe that $\xi_{s, t}^{\epsilon, 2, \natural}(\Phi)$ converges to some $\xi_{s, t}^{2, \natural}(\phi)$ because all of the other terms in (5.5) converge. In addition, the limit $\xi_{s, t}^{2, \natural}$ satisfies for all $\phi \in W^{3, \infty}$,

$$
\left|\xi_{s, t}^{2, \natural}(\phi)\right| \leq \omega_{2, \llbracket}(s, t)^{\frac{3}{p}}|\phi|_{W^{3, \infty}} .
$$

Letting $\phi \equiv 1$ in (5.6) we obtain

$$
\delta\left(|\xi|_{0}^{2}\right)_{s t}+2 \vartheta \int_{s}^{t}\left|\nabla \xi_{r}\right|_{0}^{2} d r=\left(\xi_{s}, A_{s t}^{1, *} 1+A_{s t}^{2, *} 1\right)+\xi_{s t}^{2, \natural}(1) .
$$

Because $\sigma_{k}$ are divergence-free, we deduce that $A^{1, *} 1=A^{2, *} 1=0$, which gives

$$
\delta\left(|\xi|_{0}^{2}\right)_{s t}+2 \vartheta \int_{s}^{t}\left|\nabla \xi_{r}\right|_{0}^{2} d r=\xi_{s t}^{2, \natural}(1)
$$

Summing the above equality over any partition $\pi=\left(t_{i}\right)_{i=1}^{N}$ of $[s, t]$ yields

$$
\begin{aligned}
\xi_{s t}^{2, \natural}(1) & =\delta\left(|\xi|_{0}^{2}\right)_{s t}+2 \vartheta \int_{s}^{t}\left|\nabla \xi_{r}\right|_{0}^{2} d r=\sum_{\left(t_{i}\right) \in \pi}\left(\delta\left(|\xi|_{0}^{2}\right)_{t_{i} t_{i+1}}+2 \vartheta \int_{t_{i}}^{t_{i+1}}\left|\nabla \xi_{r}\right|_{0}^{2} d r\right) \\
& =\sum_{\left(t_{i}\right) \in \pi} \xi_{t_{i-1} t_{i}}^{2, \natural}(1) \leq \sum_{t_{i} \in \pi} \omega_{2, \natural}\left(t_{i-1}, t_{i}\right)^{\frac{3}{p}} \leq \omega_{2, \natural}(s, t) \max _{i} \omega_{2, \natural}\left(t_{i-1}, t_{i}\right)^{\frac{3}{p}-1} .
\end{aligned}
$$

The above right-hand-side converges to 0 as $|\pi| \rightarrow 0$ so that $\xi^{2, \natural} \equiv 0$, proving (3.16). Moreover, this proves the continuity of $t \mapsto\left|\xi_{t}\right|_{0}^{2}$, which combined with the weak continuity $t \mapsto \xi_{t}$ in $\mathbf{H}^{-3}$ yields $\xi \in C_{T} \mathbf{H}^{0}$.

REMARK 5.7. The above shows the enstrophy balance stated in Theorem 3.10. The fact that (3.13) is also satisfied can be proved by an application of the Biot-Savart operator and Lemma 4.5.

REMARK 5.8 (Dimension three). In dimension three, we could apply the interpolation inequality $|\phi|_{L^{4}} \lesssim|\phi|_{L^{2}}^{\frac{1}{4}}|\nabla \phi|_{L^{2}}^{\frac{3}{4}}$ to prove the renormalizability of the drift of $\xi^{\otimes 2} \in$ $W^{n, 2}\left(\mathbf{T}^{3} \times \mathbf{T}^{3} ; \mathbf{R}^{3 \times 3}\right)$, and hence develop an equation for $\xi \xi^{T}$. However, this would not lead to either a global bound or energy equality because of the stretching terms. We refer the reader to the proof of existence in Section 6, where this is done for the Galerkin approximation and we do not need to double the variables.

5.2. Uniqueness. In this section, we prove that in dimension two, strong solutions of (3.1) are unique. The key idea of the proof is to derive a formula for the square of the $L^{2}$-norm of the difference of the vorticity of two arbitrary solutions. Then we show that the mean of the velocity depends continuously on the mean-free part of the velocity and the initial mean. The formula for the square can be derived in an identical fashion to the enstrophy balance in Section 5.1.

We start by showing that the mean of the velocity depends continuously on the mean-free part of the velocity and the initial mean. To see this, let $u^{(i)}, i \in\{1,2\}$, be two strong solutions 
starting from the initial conditions $u_{0}^{(i)} \in W^{1,2}$, respectively. By Remark 5.7, because $u^{(i)}$ are strong solutions, they satisfy the energy inequality

$$
\left|u^{(i)}\right|_{L_{T}^{\infty} W^{1,2}}^{2}+\int_{0}^{T}\left|\nabla^{2} u_{r}^{(i)}\right|_{0}^{2} d r \leq F\left(\left|u_{0}^{(i)}\right|_{1}\right),
$$

for $i \in\{1,2\}$ and a function $F$ as in (3.13).

Formally, $u:=u^{(1)}-u^{(2)}$ solves

$$
\partial_{t} u+\left(u^{(1)} \cdot \nabla u^{(1)}-u^{(2)} \cdot \nabla u^{(2)}\right)=\vartheta \Delta u+\left[\left(\sigma_{k} \cdot \nabla\right) u+\left(\nabla \sigma_{k}\right) u\right] \dot{z}^{k} .
$$

We understand this in the sense of Definition 3.1:

$$
\delta u_{s t}=\delta \mu_{s t}^{\Delta}+\mathcal{A}_{s t}^{1} u_{s}+\mathcal{A}_{s t}^{2} u_{s}+u_{s t}^{\natural},
$$

where for a given $\phi \in W^{1,2}$,

$$
\mu_{t}^{\Delta}(\phi):=-\int_{0}^{t}\left(\vartheta\left(\nabla u_{r}, \nabla \phi\right)+\left(u_{r}^{(1)} \cdot \nabla u_{r}^{(1)}-u_{r}^{(2)} \cdot \nabla u_{r}^{(2)}, \phi\right)\right) d r .
$$

Here, $u^{\natural}:=u^{(1), \natural}-u^{(2), \natural} \in C_{2, \omega_{Z}, L_{1} \wedge L_{2}}^{\frac{p}{3} \text {-var }}\left(\left[0, T^{*}\right] ; \mathbf{R}^{2}\right)$, where $L_{1}$ and $L_{2}$ are the constants associated with the solutions $u^{(1)}$ and $u^{(2)}$.

Denote by $v^{(i)}$ the mean-free part of $u^{(i)}, v=v^{(1)}-v^{(2)}$ and $\bar{u}=\bar{u}^{(1)}-\bar{u}^{(2)}$. We begin by deriving a bound for the mean $\bar{u}$.

LEMMA 5.9. If $u=u^{(1)}-u^{(2)}$ satisfies (5.7) in the sense of Definition 3.1, then

$$
\sup _{r \leq t}\left|\bar{u}_{r}\right| \lesssim\left|\bar{u}_{0}\right|+\sup _{r \leq t}\left|v_{r}\right|_{1}
$$

where the proportionality constant depends on $p,|\sigma|_{3, \infty}, T, \omega_{Z},\left|u_{0}^{(1)}\right|_{1}$, and $\left|u_{0}^{(2)}\right|_{1}$.

ProOF. Using the decomposition

$$
u^{(1)} \cdot \nabla u^{(1)}-u^{(2)} \cdot \nabla u^{(2)}=u^{(1)} \cdot \nabla u+u_{r} \cdot \nabla u^{(2)}=u^{(1)} \cdot \nabla v+u \cdot \nabla v^{(2)},
$$

we estimate the drift as follows:

$$
\begin{aligned}
& \frac{\left|\delta \mu_{s t}^{\Delta}\right|_{-1}}{t}-s \\
& \quad \lesssim|v|_{L^{\infty}\left([s, t] ; \mathbf{H}^{1}\right)}+\left|u^{(1)}\right|_{L^{\infty}\left([s, t] ; \mathbf{H}^{1}\right)}|v|_{L^{\infty}\left([s, t] ; \mathbf{H}^{1}\right)}+|u|_{L^{\infty}\left([s, t] ; W^{1,2)}\right.}\left|v^{(2)}\right|_{L^{\infty}\left([s, t] ; \mathbf{H}^{1}\right)} \\
& \quad \lesssim\left(1+\left|u^{(1)}\right|_{L_{\left([s, t] ; \mathbf{H}^{1}\right)}^{\infty}}+\left|u^{(2)}\right|_{\left.L_{\left([s, t] ; \mathbf{H}^{1}\right)}\right)\left(|v|_{L^{\infty}\left([s, t] ; \mathbf{H}^{1}\right)}+|\bar{u}|_{\left.L_{\left([s, t] ; \mathbf{R}^{2}\right)}\right)}\right.}\right. \\
& \quad \lesssim\left(1+F\left(\left|u_{0}^{(1)}\right|_{1}\right)+F\left(\left|u_{0}^{(2)}\right|_{1}\right)\right)\left(|v|_{L_{\left([s, t] ; \mathbf{H}^{1}\right)}}+|\bar{u}|_{L^{\infty}\left([s, t] ; \mathbf{R}^{2}\right)}\right) .
\end{aligned}
$$

Following the proof of Lemma 4.1, we find that there is a positive constant $\tilde{L}=\tilde{L}(p$, $\left.|\sigma|_{3, \infty}\right)<L_{1} \wedge L_{2}$ such that for all $(s, t) \in \Delta_{T}$ with $\omega_{Z}(s, t) \leq \tilde{L}$

$$
\left|u_{s t}^{\natural}\right|_{-1} \lesssim|u|_{L_{T}^{\infty} \mathbf{H}^{1}} \omega_{Z}(s, t)^{\frac{3}{p}}+\frac{\left|\delta \mu_{s t}^{\Delta}\right|_{-1}}{t-s}(t-s) \omega_{Z}(s, t)^{\frac{1}{3}} .
$$

Noting that

$$
\delta \bar{u}_{s t}=u_{s t}^{\natural}(e)+\left[L_{s t}^{1}+L_{s t}^{2}\right]\left(v_{s}\right),
$$

and applying the same reasoning as in the proof Lemma 4.5, we get

$$
\left|\delta \bar{u}_{s t}\right| \leq C\left(1+F\left(\left|u_{0}^{(1)}\right|_{1}\right)+F\left(\left|u_{0}^{(2)}\right|_{1}\right)\right)|\bar{u}|_{L^{\infty}\left([s, t] ; \mathbf{R}^{2}\right)} \omega_{Z}(s, t)^{\frac{1}{p}}+\phi(s, t),
$$


where we have defined

$$
\phi(s, t)=C\left(1+F\left(\left|u_{0}^{(1)}\right|_{1}\right)+F\left(\left|u_{0}^{(2)}\right|_{1}\right)\right)|v|_{L^{\infty}\left([s, t] ; \mathbf{H}^{1}\right)} \omega_{Z}(s, t)^{\frac{1}{p}} .
$$

We then obtain the desired bound by applying Lemma A.2.

Let $\xi^{(i)}=\nabla \times u^{(i)}, i \in\{1,2\}$. We now derive an estimate of $\left|\xi^{(1)}-\xi^{(2)}\right|_{0}^{2}$.

THEOREM 5.10. There is a constant $C>0$ such that for all $t \in[0, T]$,

$$
\begin{aligned}
& \sup _{\theta \leq t}\left|\xi_{\theta}^{(1)}-\xi_{\theta}^{(2)}\right|_{0}^{2}+2 \vartheta \int_{0}^{t}\left|\nabla\left(\xi_{r}^{(1)}-\xi_{r}^{(2)}\right)\right|_{0}^{2} d r \\
& \quad \leq C\left(\left|\xi_{0}^{(1)}-\xi_{0}^{(2)}\right|_{0}^{2}+\left|\bar{u}_{0}^{(1)}-\bar{u}_{0}^{(2)}\right|^{2}\right) \exp \left\{C\left|\xi_{0}^{(2)}\right|_{0}^{2}\right\} .
\end{aligned}
$$

In particular, strong solutions of (3.1) are unique.

ProOF. Formally, $\xi=\xi_{\theta}^{(1)}-\xi_{\theta}^{(2)}$ solves

$$
\partial_{t} \xi+u^{(1)} \cdot \nabla \xi^{(1)}-u^{(2)} \cdot \nabla \xi^{(2)}=\vartheta \Delta \xi+\sigma_{k} \cdot \nabla \xi \dot{z}^{k} .
$$

In the same way as in Theorem 5.6, we derive

$$
\left|\xi_{t}\right|_{0}^{2}+2 \vartheta \int_{0}^{t}\left|\nabla \xi_{r}\right|_{0}^{2} d r+\int_{0}^{t}\left(u_{r}^{(1)} \cdot \nabla \xi_{r}^{(1)}-u_{r}^{(2)} \cdot \nabla \xi_{r}^{(2)}, \xi_{r}\right) d r=\left|\xi_{0}\right|_{0}^{2}
$$

Because $u$ is divergence free, we find $\left(u_{r}^{(1)} \cdot \nabla \xi_{r}, \xi_{r}\right)=0$, so that

$$
\left(u_{r}^{(1)} \cdot \nabla \xi_{r}^{(1)}-u_{r}^{(2)} \cdot \nabla \xi_{r}^{(2)}, \xi_{r}\right)=\left(u_{r}^{(1)} \cdot \nabla \xi_{r}, \xi_{r}\right)+\left(u_{r} \cdot \nabla \xi_{r}^{(2)}, \xi_{r}\right)=-\left(u_{r} \cdot \nabla \xi_{r}, \xi_{r}^{(2)}\right) .
$$

Applying Young's inequality $a b \leq C_{\epsilon} a^{2}+\epsilon b^{2}$ and the interpolation inequality $|\cdot|_{L^{4}} \lesssim|\cdot|_{1}$ yields

$$
\begin{aligned}
\left|\xi_{t}\right|_{0}^{2}+2 \vartheta \int_{0}^{t}\left|\nabla \xi_{r}\right|_{0}^{2} d r & =\left|\xi_{0}\right|_{0}^{2}+\int_{0}^{t}\left(u_{r} \cdot \nabla \xi_{r}, \xi_{r}^{(2)}\right) d r \\
& \leq\left|\xi_{0}\right|_{0}^{2}+\int_{0}^{t}\left|u_{r}\right|_{L^{4}}\left|\xi_{r}^{(2)}\right|_{L^{4}}\left|\nabla \xi_{r}\right|_{0} d r \\
& \lesssim\left|\xi_{0}\right|_{0}^{2}+\int_{0}^{t}\left|u_{r}\right|_{1}\left|\xi_{r}^{(2)}\right|_{1}\left|\nabla \xi_{r}\right|_{0} d r \\
& \leq\left|\xi_{0}\right|_{0}^{2}+C_{\epsilon} \int_{0}^{t}\left|u_{r}\right|_{1}^{2}\left|\xi_{r}^{(2)}\right|_{1}^{2} d r+\epsilon \int_{0}^{t}\left|\nabla \xi_{r}\right|_{0}^{2} d r
\end{aligned}
$$

For $\epsilon$ small enough depending only on $\vartheta$, we get

$$
\left|\xi_{t}\right|_{0}^{2}+\vartheta \int_{0}^{t}\left|\nabla \xi_{r}\right|_{0}^{2} d r \leq\left|\xi_{0}\right|_{0}^{2}+C_{\epsilon} \int_{0}^{t}\left|u_{r}\right|_{1}^{2}\left|\xi_{r}^{(2)}\right|_{1}^{2} d r
$$

Using (2.3) and Lemma 5.9, we find

$$
\left|u_{r}\right|_{1}^{2}=\left|\bar{u}_{r}+v_{r}\right|_{0}^{2}+\left|\nabla v_{r}\right|_{0}^{2} \lesssim\left|\bar{u}_{0}\right|^{2}+\sup _{\theta \leq r}\left|v_{\theta}\right|_{1}^{2} \lesssim\left|\bar{u}_{0}\right|^{2}+\sup _{\theta \leq r}\left|\xi_{\theta}\right|_{0}^{2},
$$

which gives

$$
\sup _{\theta \leq t}\left|\xi_{\theta}\right|_{0}^{2}+\vartheta \int_{0}^{t}\left|\nabla \xi_{r}\right|_{0}^{2} d r \leq C\left(\left|\xi_{0}\right|_{0}^{2}+\left|\bar{u}_{0}\right|^{2} \int_{0}^{t}\left|\xi_{r}^{(2)}\right|_{1}^{2} d r+\int_{0}^{t}\left|\xi_{r}^{(2)}\right|_{1}^{2} \sup _{\theta \leq r}\left|\xi_{\theta}\right|_{0}^{2} d r\right) .
$$


Gronwall's inequality then implies

$$
\begin{aligned}
\sup _{\theta \leq t}\left|\xi_{\theta}\right|_{0}^{2}+\vartheta \int_{0}^{t}\left|\nabla \xi_{r}\right|_{0}^{2} d r & \leq C\left(\left|\xi_{0}\right|_{0}^{2}+\left|\bar{u}_{0}\right|^{2} \int_{0}^{t}\left|\xi_{r}^{(2)}\right|_{1}^{2} d r\right) \exp \left\{C \int_{0}^{t}\left|\xi_{r}^{(2)}\right|_{1}^{2} d r\right\} \\
& \leq C\left(\left|\xi_{0}\right|_{0}^{2}+\left|\bar{u}_{0}\right|^{2}\left|\xi_{0}^{(2)}\right|_{0}^{2}\right) \exp \left\{C\left|\xi_{0}^{(2)}\right|_{0}^{2}\right\},
\end{aligned}
$$

where we have used Theorem 5.6 for $\xi^{(2)}$ in the last inequality, and the constant $C$ may vary from line to line. This proves (5.8).

To see that this implies uniqueness of (3.2), assume $u_{0}^{(1)}=u_{0}^{(2)}$. From (5.8) we obtain $\xi^{(1)}=\xi^{(2)}$, and hence from Lemma 5.9, we find that $u^{(1)}=u^{(2)}$.

5.3. Stability. In this section, we prove Corollary 3.12. Because it is similar to the proof of Theorem 6.1 (forthcoming), we only sketch the main steps here.

Proof of Corollary 3.12. Consider a sequence $\left\{\left(u_{0}^{n}, \sigma^{n}, \mathbf{Z}^{n}\right)\right\}_{n} \in \mathbf{H}^{1} \times\left(\mathbf{W}_{\text {div }}^{3, \infty}\right)^{K} \times$ $\mathcal{C}_{g}^{p \text {-var }}\left([0, T] ; \mathbf{R}^{K}\right)$ converging to some element $\left(u_{0}, \sigma, \mathbf{Z}\right)$ in this space. By Theorem 3.10 , we have

$$
\sup _{t \in[0, T]}\left|\xi_{t}^{n}\right|_{0}^{2}+2 \vartheta \int_{0}^{T}\left|\nabla \xi_{r}^{n}\right|_{0}^{2} d r=\left|\xi_{0}^{n}\right|_{0}^{2} .
$$

Moreover, by Lemma 4.5, we can deduce the boundedness of $\left|\bar{u}^{n}\right|$. As in the proof of Theorem 6.1, we deduce that $\left\{u^{n}\right\}$ is bounded uniformly in $L_{T}^{2} \mathbf{H}^{2} \cap L_{T}^{\infty} \mathbf{H}^{1} \cap C^{p-\operatorname{var}}\left([0, T] ; \mathbf{H}^{0}\right)$, and thus there exists a subsequence, $\left\{u^{n_{k}}\right\}$ converging strongly to some $u$ in $L_{T}^{2} \mathbf{H}^{1} \cap C_{T} \mathbf{H}^{0}$. Moreover, by the assumptions on $\left\{\sigma^{n}, \mathbf{Z}^{n}\right\}_{n}$, the corresponding unbounded rough drivers, denoted by $\left(\mathcal{A}^{n, 1}, \mathcal{A}^{n, 2}\right)$, converge to $\left(\mathcal{A}^{1}, \mathcal{A}^{2}\right)$ in the strong topology; that is, $\mathcal{A}^{n, i}$ converges to $\mathcal{A}^{i}$ in the strong topology of $\mathcal{L}\left(\mathbf{H}^{k}, \mathbf{H}^{k-i}\right)$ for $i \in\{1,2\}$. Taking the limit as $n \rightarrow \infty$ then gives that $u$ satisfies (3.7). By uniqueness of solutions in dimension two, Theorem 3.10, we get that the full sequence $\left\{u^{n}\right\}$ must converge, thus showing continuity of the solution map.

Suppose now that $B$ is a Brownian motion and let $\left\{B^{n}\right\}$ denote a piecewise linear approximation of $B$. It is well known that $\left\{\left(B^{n}, \mathbb{B}^{n}\right)\right\}$ converges $\mathbb{P}$-a.s. in the rough path topology to $(B, \mathbb{B})$ where $\mathbb{B}_{s t}^{i, j}:=\int_{s}^{t} B_{s r}^{i} \circ d B_{r}^{j}$ is the Stratonovich integral. For a fixed $\phi \in \mathbf{H}^{2}$, we have as in [16], Corollary 5.2, that the rough path integral $\int_{0}^{\cdot}\left(u_{r},\left(\nabla \sigma_{k}\right) \phi-\nabla \cdot\left(\sigma_{k} \phi\right)\right) d \mathbf{B}_{r}$ and the Stratonovich integral $\int_{0}\left(u_{r},\left(\nabla \sigma_{k}\right) \phi-\nabla \cdot\left(\sigma_{k} \phi\right)\right) \circ d B_{r}$ coincide on a set, $\Omega_{\phi}$, of full measure. Choosing a dense subset $\left\{\phi_{l}\right\}_{l \in \mathbb{N}}$ of $\mathbf{H}^{2}$ and letting $\Omega_{0}:=\bigcap_{l \in \mathbb{N}} \Omega_{\phi_{l}}$ we see that the solutions must agree on $\Omega_{0}$. From the above continuity, we obtain the claimed Wong-Zakai result.

6. Existence. In this section, we prove existence of a strong solution as formulated in Theorem 3.7. The proof relies on a Galerkin approximation together with a compactness argument. First, we construct approximate solutions solving a Galerkin approximation of (3.1). In view of the a priori estimates from Section 4, we deduce certain uniform bounds leading to the desired compactness. The precise result is stated in Theorem 6.1 below. The passage to the limit then follows by classical arguments for all the terms, except for the remainder. Since all the other terms in the equation converge, we obtain the convergence of the remainder as well, and the limit remains a remainder due to a uniform bound following from Lemma 4.1.

For $d \in\{2,3\}$, let $\left\{h_{n}\right\}_{n=0}^{\infty}$ be the smooth eigenfunctions of the Stokes operator $-P \Delta$ on $\mathbf{T}^{d}$ with corresponding eigenvalues $\left\{\lambda_{n}\right\}_{n=0}^{\infty}$ where $\lambda_{0}=0$ (corresponding to $h_{0} \equiv$ const) and $\lambda_{n}>0$ for $n \in \mathbf{N}$. We choose the eigenfunctions $\left\{h_{n}\right\}_{n=0}^{\infty}$ such that they form an orthonormal 
basis of $\mathbf{H}^{0}$ and an orthogonal basis of $\mathbf{H}^{1}$. For a given $n \in \mathbf{N}$, define $l_{n}=\lambda_{n}^{-\frac{1}{2}} \nabla \times h_{n}$. It can easily be verified that $\left(\nabla^{\perp} f, g\right)=-(f, \nabla \times g)$ in $d=2$ and $(\nabla \times f, g)=-(f, \nabla \times g)$ in $d=3$. Thus $\left\{l_{n}\right\}_{n=1}^{\infty}$ forms an orthonormal basis of $\dot{\mathbf{H}}^{0}$ and we have

$$
\left(f, h_{n}\right) \nabla \times h_{n}=\left(\nabla \times f, l_{n}\right) l_{n} .
$$

For a given $N \in \mathbf{N}$, let $\mathbf{H}_{N}=\operatorname{span}\left(\left\{h_{0}, h_{1}, \ldots, h_{N}\right\}\right)$ and $\mathbf{L}_{N}=\operatorname{span}\left(\left\{l_{1}, \ldots, l_{N}\right\}\right)$, and define $P_{N}: \mathbf{H}^{-1} \rightarrow \mathbf{H}_{N}$ and $L_{N}: \dot{\mathbf{H}}^{-1} \rightarrow \dot{\mathbf{L}}_{N}$ by

$$
P_{N} v:=\sum_{n=0}^{N}\left(v, h_{n}\right) h_{n}, \quad L_{N} v:=\sum_{n=1}^{N}\left(v, l_{n}\right) l_{n}, \quad v \in \mathbf{H}^{-1} .
$$

It follows from (6.1) that

$$
\nabla \times P_{N} v=L_{N}(\nabla \times v) \quad \forall v \in \mathbf{H}^{-1} .
$$

Since $\mathbf{Z} \in C_{g}^{p \text {-var }}\left([0, T] ; \mathbf{R}^{K}\right)$ is a geometric rough path, there is a sequence of $\mathbf{R}^{K}$-valued smooth paths $\left\{z^{N}\right\}_{N=1}^{\infty}$ such that their canonical lifts $\mathbf{Z}^{N}=\left(Z^{N}, \mathbb{Z}^{N}\right)$ converge to $\mathbf{Z}$ in the rough path topology. We assume that

$$
\left|Z_{s t}^{N}\right| \lesssim \omega_{Z}(s, t)^{\frac{1}{p}}, \quad\left|\mathbb{Z}_{s t}^{N}\right| \lesssim \omega_{Z}(s, t)^{\frac{2}{p}} \quad \forall(s, t) \in \Delta_{T} .
$$

Let us consider the following $N$ th order Galerkin approximation of (3.1) in $\mathbf{H}_{N}$ :

$$
\partial_{t} u^{N}+P_{N} B_{P}\left(u^{N}\right)=\vartheta P_{N} \Delta u^{N}+\sum_{k=1}^{K} P_{N} P\left[\left(\sigma_{k} \cdot \nabla\right) u^{N}+\left(\nabla \sigma_{k}\right) u^{N}\right] \dot{z}_{t}^{N, k},
$$

with initial condition $u^{N}(0)=P_{N} u_{0}$. This is a system of ODEs in $\mathbf{H}_{N}$ with locally Lipschitz coefficients, and consequently there exists a time $T_{N}>0$ and a unique solution $u^{N}$ of (6.4) on the interval $\left[0, T_{N}\right)$.

Integrating (6.4) over the interval $[s, t]$, we find

$$
\delta u_{s t}^{N}=\int_{s}^{t}\left(\vartheta P_{N} \Delta u_{r}^{N}-P_{N} B_{P}\left(u_{r}^{N}\right)\right) d r+\mathcal{A}_{s t}^{N, 1} u_{s}^{N}+\mathcal{A}_{s t}^{N, 2} u_{s}^{N}+u_{s t}^{N, \natural},
$$

where $\tilde{P}_{N}:=P_{N} P$, and $\mathcal{A}_{s t}^{N, i}$ and $u_{s t}^{N, \natural}$ are defined as in (3.4) and (3.5), respectively, with $P$ replaced by $\tilde{P}_{N}$ and $\mathbf{Z}$ replaced by $\mathbf{Z}^{N}$.

Owing to (2.2) and (6.3), we find that $\left(\mathcal{A}^{N, 1}, \mathcal{A}^{N, 2}\right)$ is uniformly bounded in $N$ as a family of unbounded rough drivers on the scale $\left(\mathbf{H}^{n}\right)_{n}$. That is, there exists a control $\omega_{\mathcal{A}^{N}}$ such that (2.7) holds and for all $(s, t) \in \Delta_{T}$,

$$
\omega_{\mathcal{A}^{N}}(s, t) \lesssim|\sigma|_{3, \infty} \omega_{Z}(s, t) .
$$

Estimating term-by-term and using (2.2), (2.4), and that $u^{N}$ is smooth in space, and $z^{N}$ is smooth in time, we find that $u^{N, \natural} \in C_{2}^{\frac{p}{3} \text {-var }}\left(\left[0, T_{N}\right) ; \mathbf{H}_{N}\right)$. Arguing as in Lemma 4.1, we get that there is an $L=L\left(p,|\sigma|_{3, \infty}\right)>0$ such that for all $(s, t) \in \Delta_{T_{N}}$ with $\omega_{Z}(s, t) \leq L$,

$$
\begin{aligned}
\omega_{N, \natural}(s, t) & :=\left|u^{N, \natural \mid}\right|_{\frac{p}{3}-\operatorname{var} ;[s, t] ; \mathbf{H}^{-2}}^{\frac{p}{3}} \\
& \lesssim p,|\sigma|_{3, \infty}\left|u^{N}\right|_{L_{T_{N}}^{\infty} \mathbf{H}^{1}}^{\frac{p}{3}} \omega_{Z}(s, t)+\left(1+\left|u^{N}\right|_{L_{T_{N}}^{\infty} \mathbf{H}^{1}}\right)^{\frac{2 p}{3}}(t-s)^{\frac{p}{3}} \omega_{Z}(s, t)^{\frac{1}{3}} .
\end{aligned}
$$

In order to obtain uniform energy bounds on $u^{N}$ independent of $N$, we will obtain a uniform bound on the vorticity $\xi^{N}:=\nabla \times u^{N}$ and the mean $\bar{u}^{N}:=\int_{\mathbf{T}^{d}} u^{N} d x$. Let

$$
v^{N}=u^{N}-\bar{u}^{N}=\mathcal{K} \xi^{N} \text {. }
$$


By an analogue of Lemma 4.5 applied to the Galerkin approximation and on the sub-interval $[0, t] \subset\left[0, T_{N}\right)$, there is a positive constant $C=C\left(p,|\sigma|_{3, \infty}, T_{N}, \omega_{Z}\right)$ such that

$$
\sup _{0 \leq r \leq t}\left|\bar{u}_{r}^{N}\right| \leq C \exp \left\{C\left(1+\sup _{0 \leq r \leq t}\left|v_{r}^{N}\right|_{\mathbf{H}^{1}}\right)^{p}\right\}\left(1+\left|\bar{u}_{0}\right|\right) .
$$

Using (2.3) and Poincare's inequality, we find $\left|v^{N}\right|_{0} \lesssim\left|\nabla v^{N}\right|_{0}=\left|\xi^{N}\right|_{0}$, and thus $\left|v^{N}\right|_{1} \lesssim$ $\left|\xi^{N}\right|_{0}$. Thus, if we obtain a uniform bound on $\left|\xi^{N}\right|_{L_{T_{N}}^{\infty} \mathbf{H}^{0}}$, then we can obtain a uniform bound on $\left|\bar{u}^{N}\right|_{L_{T_{N}}^{\infty} \mathbf{R}^{d}}$, which can then be used to derive a uniform bound on $u^{N}=v^{N}+\bar{u}^{N}$. We now proceed by obtaining uniform bounds on $\xi^{N}$.

Using properties of the curl operator in Section 2.1 and the relation (6.2), we get that $\xi^{N}$ satisfies

$$
\partial_{t} \xi^{N}+£_{u^{N}}^{N} \xi^{N}=\vartheta L_{N} \Delta \xi^{N}+£_{\sigma_{k}}^{N} \xi^{N} \dot{z}_{t}^{N, k}
$$

where

$$
\mathfrak{£}_{v} \phi=(v \cdot \nabla) \phi-\mathbf{1}_{d=3}(\phi \cdot \nabla) v, \quad £_{v}^{N}=L_{N} £_{v} .
$$

Deriving uniform bounds in dimension two is the simplest due to the conservative nature of the equation. However, this is no longer possible in dimension three because there is an additional stretching term in the drift and a lower order term in the noise that forces us to use a nonlinear version of the rough Gronwall's inequality, Lemma A.4.

We begin with the dimension two case. Testing (6.9) by $\xi^{N}$ and using (2.5), integration by parts and the fact that $\nabla \cdot \sigma_{k}=0$, for all $k \in\{1, \ldots, K\}$, we obtain that for all $t \in\left[0, T_{N}\right)$,

$$
\begin{aligned}
\left|\xi_{t}^{N}\right|_{0}^{2}+2 \vartheta \int_{0}^{t}\left|\nabla \xi_{s}^{N}\right|_{0}^{2} d s= & \left|L_{N} \xi_{0}\right|_{0}^{2}-2 \int_{0}^{t}\left(\left(u_{s}^{N} \cdot \nabla\right) \xi_{s}^{N}, \xi_{s}^{N}\right) d s \\
& +2 \int_{0}^{t}\left(\left(\sigma_{k} \cdot \nabla\right) \xi_{s}^{N}, \xi_{s}^{N}\right) d z_{s}^{N, k} \\
\leq & \left|\xi_{0}\right|_{0}^{2} .
\end{aligned}
$$
Thus,

Owing to the Poincaré inequality and (2.3) we have $\left|v^{N}\right|_{0} \lesssim\left|\xi^{N}\right|_{0}$ and $\left|\nabla^{2} v^{N}\right|_{0}=\left|\nabla \xi^{N}\right|_{0}$.

$$
\left|v_{t}^{N}\right|_{1}^{2}+2 \vartheta \int_{0}^{t}\left|\nabla^{2} u_{s}^{N}\right|_{0}^{2} d s \lesssim\left|\nabla u_{0}\right|_{0}^{2} \quad \forall t \in\left[0, T_{N}\right) .
$$

We now turn our attention to dimension three. As mentioned above, it is not possible to obtain an enstrophy bound independent of the noise approximation like we did in twodimensions because the noise is not conservative due to the presence of the stretching term $\left(\xi^{N} \cdot \nabla\right) \sigma_{k}$. Integrating (6.9) over the interval $[s, t]$, we obtain

$$
\delta \xi_{s t}^{N}=\delta \gamma_{s t}^{N}+\left[A_{s t}^{N, 1}+A_{s t}^{N, 2}\right] \xi_{s}^{N}+\xi_{s t}^{N, \natural}
$$

where

$$
\begin{aligned}
\gamma_{t}^{N} & :=\int_{0}^{t}\left(\vartheta L_{N} \Delta \xi_{r}^{N}-£_{u_{r}^{N}}^{N} \xi_{r}^{N}\right) d r, \quad A_{s t}^{N, 1} \phi:=£_{\sigma_{k}}^{N} \phi Z_{s t}^{N, k}, \quad A_{s t}^{N, 2} \phi:=£_{\sigma_{k}}^{N} £_{\sigma_{l}}^{N} \phi \mathbb{Z}_{s t}^{l, k}, \\
\xi_{s t}^{N, \natural} & :=\int_{s}^{t} £_{\sigma_{k}}^{N} \delta \gamma_{s r}^{N} d z_{r}^{N, k}+\int_{s}^{t} \int_{s}^{r} £_{\sigma_{k}}^{N} £_{\sigma_{l}}^{N}\left[\delta \gamma_{s r_{1}}^{N}+\int_{s}^{r} £_{\sigma_{m}}^{N} \xi_{r_{2}}^{N} d z_{r_{2}}^{N, m}\right] d z_{r_{1}}^{N, l} d z_{r}^{N, k} .
\end{aligned}
$$

In order to distinguish from the notation used for the doubling of variables in Section 5, we use $\otimes$ to denote the standard tensor product on $\mathbf{R}^{3}$. We proceed by deriving the equation for

$$
\Xi^{N}=\xi^{N} \underline{\xi^{N}}=\left[\xi^{N, i} \xi^{N, j}\right]_{1 \leq i, j \leq 3} \text {. }
$$


Defining the symmetric tensor $a \underline{\hat{\otimes}} b:=\frac{1}{2}(a \underline{\otimes} b+b \underline{\otimes} a)$, we have $\delta \Xi_{s t}^{N}=2 \xi_{s}^{N} \underline{\hat{\otimes}} \delta \xi_{s t}^{N}+$ $\left(\delta \xi_{s t}^{N}\right) \underline{\otimes 2}$, and hence

$$
\delta \Xi_{s t}^{N}=\delta \Pi_{s t}^{N}+\left[\Gamma_{s t}^{N, 1}+\Gamma_{s t}^{N, 2}\right] \Xi_{s}^{N}+\Xi_{s t}^{N, \natural}
$$

where

$$
\begin{aligned}
\Pi_{t}^{N}:= & 2 \int_{0}^{t} \xi_{r}^{N} \underline{\hat{\otimes}}\left(\vartheta L_{N} \Delta \xi_{r}^{N}-£_{u_{r}^{N}}^{N} \xi_{r}^{N}\right) d r, \\
\Gamma_{s t}^{N, 1} \Xi_{s}^{N}:= & \left(2 A_{s t}^{N, 1} \xi_{s}^{N}\right) \underline{\hat{\otimes}} \xi_{s}^{N}, \quad \Gamma_{s t}^{N, 2} \Xi_{s}^{N}:=\left(2 A_{s t}^{N, 2} \xi_{s}^{N}\right) \underline{\hat{\otimes}} \xi_{s}^{N}+\left(A_{s t}^{N, 1} \xi_{s}^{N}\right) \underline{\hat{\otimes}}\left(A_{s t}^{N, 1} \xi_{s}^{N}\right), \\
\Xi_{s t}^{N, \natural}= & 2 \xi_{s}^{N} \underline{\hat{\otimes}} \xi_{s t}^{N, \natural}-2 \int_{s}^{t} \delta \xi_{s r}^{N} \underline{\hat{\otimes}}\left(\vartheta L_{N} \Delta \xi_{r}^{N}-£_{u_{r}^{N}} \xi_{r}^{N}\right) d r+\left(\delta \xi_{s t}^{N}\right)^{\underline{\otimes} 2} \\
& -\left(A_{s t}^{N, 1} \xi_{s}^{N}\right) \underline{\hat{\otimes}}\left(A_{s t}^{N, 1} \xi_{s}^{N}\right) .
\end{aligned}
$$

By virtue of (2.1) and (6.3), $\left(\Gamma^{N, 1}, \Gamma^{N, 2}\right)$ is uniformly bounded in $N$ as a family of unbounded rough drivers on the scale $\left(\dot{\mathbf{W}}^{n, \infty}\left(\mathbf{T}^{3} ; \mathbf{R}^{3 \times 3}\right)\right)_{n}$. That is, there exists a control $\omega_{\Gamma^{N}}$ such that (2.7) holds and for all $(s, t) \in \Delta_{T}$,

$$
\omega_{\Gamma^{N}}(s, t) \lesssim|\sigma|_{3, \infty} \omega_{Z}(s, t) .
$$

For ease of notation, let $|\cdot|_{\infty}=|\cdot|_{0, \infty}$. To find a control for $\Pi^{N}$, we need to estimate

$$
\Pi_{s t}^{N}(\Phi)=2 \int_{s}^{t} \xi_{r}^{N} \underline{\hat{\otimes}} \vartheta L_{N} \Delta \xi_{r}^{N}(\Phi) d r-2 \int_{s}^{t} \xi_{r}^{N} \underline{\hat{\otimes}} \xi_{u_{r}^{N}}^{N} \xi_{r}^{N}(\Phi) d r=: I+I I .
$$

Applying Young's inequality, we find

$$
\begin{aligned}
I & =-2 \vartheta \int_{s}^{t} \int_{\mathbf{T}^{3}} \partial_{l} \xi_{r}^{N} \underline{\hat{\otimes}} \partial_{l} \xi_{r}^{N}(\Phi) d x d r-2 \vartheta \int_{s}^{t} \int_{\mathbf{T}^{3}} \xi_{r}^{N} \underline{\hat{\otimes}} \partial_{l} \xi_{r}^{N}\left(\partial_{l} \Phi\right) d x d r \\
& \leq 2 \vartheta|\Phi|_{1, \infty}\left(\int_{s}^{t}\left|\nabla \xi_{r}^{N}\right|_{0}^{2} d r+\int_{s}^{t} \sup _{0 \leq r^{\prime} \leq r}\left|\xi_{r^{\prime}}^{N}\right|_{0}^{2} d r\right) .
\end{aligned}
$$

We split $I I$ into two quantities $I I I$ and $I V$ and then estimate them separately:

$$
\begin{aligned}
-I I & =2 \int_{s}^{t} \int_{\mathbf{T}^{3}} \xi_{r}^{N} \underline{\hat{\otimes}}\left[\left(u_{r}^{N} \cdot \nabla\right) \xi_{r}^{N}\right](\Phi) d x d r+2 \int_{s}^{t} \int_{\mathbf{T}^{3}} \xi_{r}^{N} \underline{\hat{\otimes}}\left[\left(\nabla u_{r}^{N}\right) \xi_{r}^{N}\right](\Phi) d x d r \\
& =: I I I+I V .
\end{aligned}
$$

Using the interpolation inequality $|f|_{L^{4}} \leq C_{3}|f|_{0}^{\frac{1}{4}}|\nabla f|_{0}^{\frac{3}{4}}$ for $d=3$, Hölder's, Young's and Poincaré's inequality, (6.8), and (2.3), we get

$$
\begin{aligned}
I I I & \lesssim|\Phi|_{\infty} \int_{s}^{t}\left|\xi_{r}^{N}\right|_{0}^{\frac{1}{4}}\left|\nabla \xi_{r}^{N}\right|_{0}^{\frac{3}{4}}\left|u_{r}^{N}\right|_{0}^{\frac{1}{4}}\left|\nabla u_{r}^{N}\right|_{0}^{\frac{3}{4}}\left|\nabla \xi_{r}^{N}\right|_{0} d r \\
& \lesssim|\Phi|_{\infty} \int_{s}^{t}\left|\xi_{r}^{N}\right|_{0}\left|u_{r}^{N}\right|_{0}^{\frac{1}{4}}\left|\nabla \xi_{r}^{N}\right|_{0}^{7 / 4} d r \\
& \lesssim|\Phi|_{\infty}\left(\int_{s}^{t}\left|u_{r}^{N}\right|_{0}^{2}\left|\xi_{r}^{N}\right|_{0}^{8} d r+\int_{s}^{t}\left|\nabla \xi_{r}^{N}\right|_{0}^{2} d r\right) \\
& \lesssim|\Phi|_{\infty}\left(\int_{s}^{t}\left|\bar{u}_{r}^{N}\right|^{2}\left|\xi_{r}^{N}\right|_{0}^{8} d r+\int_{s}^{t}\left|\xi_{r}^{N}\right|_{0}^{10} d r+\int_{s}^{t}\left|\nabla \xi_{r}^{N}\right|_{0}^{2} d r\right) \\
& \lesssim|\Phi|_{\infty}\left(\int_{s}^{t} w_{1}\left(\sup _{0 \leq r^{\prime} \leq r}\left|\xi_{r^{\prime}}^{N}\right|_{0}\right) d r+\int_{s}^{t}\left|\nabla \xi_{r}^{N}\right|_{0}^{2} d r\right),
\end{aligned}
$$


where

$$
w_{1}(y):=\left(1+\left|\bar{u}_{0}\right|\right) \exp \left\{C(1+y)^{p}\right\} y^{8}+y^{10}
$$

Similarly, we find

$$
\begin{aligned}
I V & \lesssim|\Phi|_{\infty} \int_{s}^{t} \int_{\mathbf{T}^{3}}\left|\xi_{r}^{N}\right|_{0}^{\frac{1}{2}}\left|\nabla \xi_{r}^{N}\right|_{0}^{\frac{6}{4}}\left|\nabla u_{r}^{N}\right|_{0} d r d x \lesssim|\Phi|_{\infty} \int_{s}^{t}\left|\xi_{r}^{N}\right|_{0}^{\frac{3}{2}}\left|\nabla \xi_{r}^{N}\right|_{0}^{\frac{6}{4}} d r \\
& \lesssim|\Phi|_{\infty}\left(\int_{S}^{t} \sup _{0 \leq r^{\prime} \leq r}\left|\xi_{r^{\prime}}^{N}\right|_{0}^{6} d r+\int_{s}^{t}\left|\nabla \xi_{r}^{N}\right|_{0}^{2} d r\right) .
\end{aligned}
$$

Therefore,

$$
\left|\Pi_{s t}^{N}\right|_{-1, \infty} \lesssim \omega_{\Pi^{N}}(s, t):=\int_{s}^{t}\left|\nabla \xi_{r}^{N}\right|_{0}^{2} d r+\int_{s}^{t} w_{2}\left(\sup _{0 \leq r^{\prime} \leq r}\left|\xi_{r^{\prime}}^{N}\right|_{0}\right) d r
$$

where

$$
w_{2}(y):=\left(1+\left|\bar{u}_{0}\right|\right) \exp \left\{C(1+y)^{p}\right\} y^{8}+y^{10}+y^{6}+y^{2} .
$$

By Theorem 2.6, there is a constant $L=L\left(p,|\sigma|_{3, \infty}\right)>0$ such that for all $(s, t) \in \Delta_{T_{N}}$ with $\omega_{Z}(s, t) \leq L$,

$$
\begin{aligned}
\left|\Xi_{s t}^{N, \natural}\right|_{-3, \infty} \lesssim & \sup _{s \leq r \leq t}\left|\Xi_{r}^{N}\right|_{-0, \infty} \omega_{Z}(s, t)^{\frac{3}{p}}+\omega_{\Pi^{N}}(s, t) \omega_{Z}(s, t)^{\frac{1}{p}} \\
\lesssim & \sup _{s \leq r \leq t}\left|\xi_{r}^{N}\right|_{0}^{2} \omega_{Z}(s, t)^{\frac{3}{p}}+\omega_{Z}(s, t)^{\frac{1}{p}} \int_{s}^{t}\left|\nabla \xi_{r}^{N}\right|_{0}^{2} d r \\
& +\omega_{Z}(s, t)^{\frac{1}{p}} \int_{s}^{t} w_{2}\left(\sup _{0 \leq r^{\prime} \leq r}\left|\xi_{r^{\prime}}^{N}\right|_{0}\right) d r .
\end{aligned}
$$

Testing (6.11) against the $3 \times 3$ identity matrix $I_{3}$, we find

$$
\delta\left(\left|\xi^{N}\right|_{0}^{2}\right)_{s t}=\delta \Pi_{s t}^{N}\left(I_{3}\right)+\Xi_{s}^{N}\left(\left[\Gamma_{s t}^{N, 1, *}+\Gamma_{s t}^{N, 2, *}\right]\left(I_{3}\right)\right)+\Xi_{s t}^{N, \natural}\left(I_{3}\right) .
$$

It follows from (6.12) that

$$
\left|\Xi_{s}^{N}\left(\left[\Gamma_{s t}^{N, 1, *}+\Gamma_{s t}^{N, 2, *}\right]\left(I_{3}\right)\right)\right| \lesssim \sup _{s \leq r \leq t}\left|\xi_{r}^{N}\right|_{0}^{2} \omega_{Z}(s, t)^{\frac{1}{p}}
$$

Applying Hölder's inequality, the interpolation inequality $|f|_{L^{4}} \lesssim C_{3}|f|_{0}^{\frac{1}{4}}|\nabla f|_{0}^{\frac{3}{4}}$ for $d=3$ and Young's inequality $a b \leq C_{\epsilon} a^{4}+\epsilon b^{\frac{4}{3}}$ for $\epsilon \in(0,1)$ to be determined later, we get

$$
\begin{aligned}
\delta \Pi_{s t}^{N}\left(I_{3}\right) & =-2 \vartheta \int_{s}^{t}\left|\nabla \xi_{r}^{N}\right|_{0}^{2} d r+\int_{s}^{t} \int_{\mathbf{T}^{3}} \xi_{r}^{N, i} \partial_{i} u_{r}^{N, j} \xi_{r}^{N, j} d x d r \\
& \leq-2 \vartheta \int_{s}^{t}\left|\nabla \xi_{r}^{N}\right|_{0}^{2} d r+\int_{s}^{t}\left|\xi_{r}^{N}\right|_{L^{4}}^{2}\left|\nabla u_{r}^{N}\right|_{0} d r \\
& \leq-2 \vartheta \int_{s}^{t}\left|\nabla \xi_{r}^{N}\right|_{0}^{2} d r+C_{3} \int_{s}^{t}\left|\xi_{r}^{N}\right|_{0}^{\frac{3}{2}}\left|\nabla \xi_{r}^{N}\right|_{0}^{\frac{3}{2}} d r \\
& \leq-\left(2 \vartheta-C_{3} \epsilon\right) \int_{s}^{t}\left|\nabla \xi_{r}^{N}\right|_{0}^{2} d r+C_{\epsilon} C_{3} \int_{s}^{t} \sup _{0 \leq r^{\prime} \leq r}\left|\xi_{r^{\prime}}^{N}\right|_{0}^{6} d r .
\end{aligned}
$$

Putting it all together, we arrive at

$$
\begin{aligned}
\delta\left(\left|\xi^{N}\right|_{0}^{2}\right)_{s t} \leq & {\left[C \omega_{Z}(s, t)^{\frac{1}{p}}-\left(2 \vartheta-C_{3} \epsilon\right)\right] \int_{s}^{t}\left|\nabla \xi_{r}^{N}\right|_{0}^{2} d r } \\
& +C \int_{s}^{t} w\left(\sup _{0 \leq r^{\prime} \leq r}\left|\xi_{r^{\prime}}^{N}\right|_{0}\right) d r+C \sup _{s \leq r \leq t}\left|\xi_{r}\right|_{0}^{2} \omega_{Z}(s, t)^{\frac{1}{p}}
\end{aligned}
$$


where

$$
w(y)=\left(1+\left|\bar{u}_{0}\right|\right) \exp \left\{C(1+y)^{p}\right\} y^{8}+y^{10}+\left(1+C_{3} C_{\epsilon}\right) y^{6}+y^{2} .
$$

If we let $\epsilon=\frac{\vartheta}{C_{3}}$, then for all $(s, t) \in \Delta_{T_{N}}$ such that $C \omega_{Z}(s, t)^{\frac{1}{p}} \leq 2 \vartheta=\vartheta+C_{3} \epsilon$ and $\omega_{Z}(s, t) \leq L$, we get $C \omega_{Z}(s, t)^{\frac{1}{p}}-\left(2 \vartheta-C_{3} \epsilon\right) \leq-\vartheta$, which gives

$$
\delta\left(\left|\xi^{N}\right|_{0}^{2}\right)_{s t}+\vartheta \int_{s}^{t}\left|\nabla \xi_{r}^{N}\right|_{0}^{2} d r \leq C \int_{s}^{t} w\left(\sup _{0 \leq r^{\prime} \leq r}\left|\xi_{r^{\prime}}^{N}\right|_{0}\right) d r+\sup _{s \leq r \leq t}\left|\xi_{r}\right|_{0}^{2} C \omega_{Z}(s, t)^{\frac{1}{p}} .
$$

Applying Lemma A.4 on $\left[0, T_{N}\right)$, we obtain

$$
\sup _{0 \leq r \leq T^{*} \wedge T_{N}}\left|\xi_{r}^{N}\right|_{0}^{2} \leq W^{-1}\left(W\left(q\left|\xi_{0}^{N}\right|_{0}^{2}\right)+T^{*} C K q\right)
$$

where $T^{*}=T^{*}\left(p, \omega_{Z},|\sigma|_{3, \infty},\left|u_{0}\right|_{1}\right)>0$ is such that

$$
W\left(q\left|\xi_{0}^{N}\right|_{0}^{2}\right)+T^{*} C K q \in \operatorname{Dom}\left(W^{-1}\right)
$$

and $W, q$ and $K$ are specified in Lemma A.4. We emphasize that $T^{*}$ can be chosen independently of $N$. It follows from Poincaré inequality and (2.3) that there is a continuous increasing function $\tilde{F}: \mathbf{R}_{+} \rightarrow \mathbf{R}_{+}$such that

$$
\sup _{t \in\left[0, T^{*} \wedge T_{N}\right]}\left|v_{t}^{N}\right|_{1}^{2}+\vartheta \int_{0}^{T^{*} \wedge T_{N}}\left|\nabla^{2} u_{s}^{N}\right|_{0}^{2} d s \leq \tilde{F}\left(\left|\nabla u_{0}\right|_{0}\right) .
$$

We now conclude with uniform bounds on $u^{N}$ for $d \in\{2,3\}$. In dimension two, by (6.8) and (6.10), there is a continuous increasing function $F: \mathbf{R}_{+} \rightarrow \mathbf{R}_{+}$such that

$$
\sup _{t \in\left[0, T_{N}\right]}\left|u_{t}^{N}\right|_{1}^{2}+\int_{0}^{T_{N}}\left|\nabla^{2} u_{r}^{N}\right|_{0}^{2} d r \leq F\left(\left|u_{0}\right|_{1}\right), \quad d=2
$$

and hence the solution $u^{N}$ can be extended to the interval $[0, T]$ in a standard way. In dimension three, by (6.8) and (6.13), there is a continuous increasing function $F: \mathbf{R}_{+} \rightarrow \mathbf{R}_{+}$such that

$$
\sup _{t \in\left[0, T^{*} \wedge T_{N}\right]}\left|u_{t}^{N}\right|_{1}^{2}+\int_{0}^{T^{*} \wedge T_{N}}\left|\nabla^{2} u_{r}^{N}\right|_{0}^{2} d r \leq F\left(\left|u_{0}\right|_{1}\right), \quad d=3,
$$

and hence the solution $u^{N}$ can be extended to the interval [0, $\left.T^{*}\right]$.

We are now ready to let $N \rightarrow \infty$ in (6.5), which will complete the proof of Theorem 3.7. The details are given in the following theorem. Throughout the rest of this section, let $T^{*}=T$ if $d=2$ and $T^{*}>0$ be the final time obtained by means of Lemma A. 4 above if $d=3$

THEOREM 6.1. There exists a subsequence of $\left\{u^{N}\right\}$ that converges weakly in $L_{T^{*}}^{2} \mathbf{H}^{2}$, weak-* in $L_{T^{*}}^{\infty} \mathbf{H}^{1}$, and strongly in $L_{T^{*}}^{2} \mathbf{H}^{1} \cap C_{T^{*}} \mathbf{H}^{0}$ to a solution of (3.7) that is weakly continuous in $\mathbf{H}^{1}$.

PROOF. We have shown above that

$$
\sup _{t \in\left[0, T^{*}\right]}\left|u_{t}^{N}\right|_{1}^{2}+\int_{0}^{T^{*}}\left|\nabla^{2} u_{r}^{N}\right|_{0}^{2} d r \leq F\left(\left|u_{0}\right|_{1}\right)
$$

for a continuous increasing function $F: \mathbf{R}_{+} \rightarrow \mathbf{R}_{+}$. Thus, $\left\{u^{N}\right\}$ remains in a bounded set in $L_{T^{*}}^{2} \mathbf{H}^{2} \cap L_{T^{*}}^{\infty} \mathbf{H}^{1}$. The Banach-Alaoglu theorem yields a subsequence, which we relabel 
as $\left\{u^{N}\right\}$, that converges weakly in $L_{T^{*}}^{2} \mathbf{H}^{2}$ and weak-* in $L_{T^{*}}^{\infty} \mathbf{H}^{1}$. Moreover, by (6.7), for all $(s, t) \in \Delta_{T_{N}}$ with $\omega_{Z}(s, t) \leq L$,

$$
\omega_{N, \sharp}(s, t) \lesssim_{p,|\sigma|_{3, \infty}} \tilde{F}\left(\left|u_{0}\right|_{1}\right)\left(\omega_{Z}(s, t)+(t-s)^{\frac{p}{3}} \omega_{Z}(s, t)^{\frac{1}{3}}\right),
$$

for some continuous increasing function $\tilde{F}: \mathbf{R}_{+} \rightarrow \mathbf{R}_{+}$.

We will obtain a further subsequence that converges strongly in $L_{T^{*}}^{2} \mathbf{H}^{1} \cap C_{T^{*}} \mathbf{H}^{0}$ by applying a rough version of the Aubin-Lions compactness lemma. We refer the reader to [21], Lemma A.2, and note that we actually use the lemma shifted up by one. By (6.6), (6.14) and Lemma 4.3 , there is a positive constant $\tilde{L}=\tilde{L}\left(p,|\sigma|_{3, \infty}\right)<L$ such that for all $(s, t) \in \Delta_{T^{*}}$ with $(t-s)+\omega_{Z}(s, t) \leq \tilde{L}$, it holds that

$$
\left|\delta u_{s t}^{N}\right|_{0} \lesssim_{p,|\sigma|_{3, \infty}} \tilde{F}\left(\left|u_{0}\right|_{1}\right)\left((t-s)+\omega_{Z}(s, t)+(t-s)^{\frac{p}{3}} \omega_{Z}(s, t)^{\frac{1}{3}}\right)^{\frac{1}{p}},
$$

for some continuous increasing function $\tilde{F}: \mathbf{R}_{+} \rightarrow \mathbf{R}_{+}$. It then follows from the aforementioned Aubin-Lions compactness result that there is a subsequence of $\left\{u^{N}\right\}$, which we continue to denote by $\left\{u^{N}\right\}$, converging strongly to an element $u$ in $C_{T^{*}} \mathbf{H}^{0} \cap L_{T^{*}}^{2} \mathbf{H}^{1}$. Furthermore, owing to Lemma [21], Lemma A.3 (shifted one space up), we know that $u$ is continuous with values in $\mathbf{H}_{w}^{1}$ (i.e., $\mathbf{H}^{1}$ equipped with the weak topology).

Our goal now is to pass to the limit as $N \rightarrow \infty$ of (6.5) tested against some $\phi \in \mathbf{H}^{2}$. Using the definition of $\mathcal{A}_{s t}^{N, 1}$ and $\mathcal{A}_{s t}^{1}$, we find

$$
\begin{aligned}
\mid \mathcal{A}_{s t}^{N, 1} & \phi-\left.\mathcal{A}_{s t}^{1} \phi\right|_{0} \\
\leq & \left|P_{N} P\left[\left(\sigma_{k} \cdot \nabla\right) \phi\right] Z_{s t}^{N, k}-P\left[\left(\sigma_{k} \cdot \nabla\right) \phi\right] Z_{s t}^{k}\right|_{0} \\
& +\left|P_{N} P\left[\left(\nabla \sigma_{k}\right) \phi\right] Z_{s t}^{N, k}-P\left[\left(\nabla \sigma_{k}\right) \phi\right] Z_{s t}^{k}\right|_{0} \\
\leq & \left|P_{N} P\left[\left(\sigma_{k} \cdot \nabla\right) \phi\right]\right|_{0}\left|Z_{s t}^{N, k}-Z_{s t}^{k}\right|+\left|P_{N} P\left[\left(\nabla \sigma_{k}\right) \phi\right]\right|_{0}\left|Z_{s t}^{N, k}-Z_{s t}^{k}\right| \\
& +\left|\left(I-P_{N}\right) P\left[\left(\sigma_{k} \cdot \nabla\right) \phi\right]\right|_{0}\left|Z_{s t}^{k}\right|+\left|\left(I-P_{N}\right) P\left[\left(\nabla \sigma_{k}\right) \phi\right]\right|_{0}\left|Z_{s t}^{k}\right| .
\end{aligned}
$$

From (2.2), we obtain

$$
\begin{gathered}
\left|P_{N} P\left(\sigma_{k} \cdot \nabla\right) \phi\right|_{0}\left|Z_{s t}^{N, k}-Z_{s t}^{k}\right| \lesssim_{N_{0}}|\phi|_{1}\left|Z_{s t}^{N}-Z_{s t}\right|, \\
\left|P_{N} P\left(\nabla \sigma_{k}\right) \phi\right|_{0}\left|Z_{s t}^{N, k}-Z_{s t}^{k}\right| \lesssim N_{0}|\phi|_{0}\left|Z_{s t}^{N}-Z_{s t}\right|,
\end{gathered}
$$

which both converge to 0 as $N \rightarrow \infty$. Making use of (2.2) again, the last two terms of (6.16) tend to 0 as $N \rightarrow \infty$ because $P_{N}$ converges to the identity $I$ in the strong topology on $\mathcal{L}\left(\mathbf{H}^{0}, \mathbf{H}^{0}\right)$. Thus, for all $\phi \in \mathbf{H}^{2}, \lim _{N \rightarrow \infty}\left|\mathcal{A}_{s t}^{N, 1} \phi-\mathcal{A}_{s t}^{1} \phi\right|_{0}=0$. In a similar way, we deduce that $\lim _{N \rightarrow \infty}\left|\mathcal{A}_{s t}^{N, 2} \phi-\mathcal{A}_{s t}^{2} \phi\right|_{0}=0$ for all $\phi \in \mathbf{H}^{2}$. Therefore,

$$
\begin{aligned}
\left|\left(u_{s}^{N}, \mathcal{A}_{s t}^{N, i, *} \phi\right)-\left(u_{s}, \mathcal{A}_{s t}^{P, i, *} \phi\right)\right| & \leq_{N_{0}}\left|\left(u_{s}^{N}-u_{s}, \mathcal{A}_{s t}^{N, i, *} \phi\right)-\left(u_{s},\left(\mathcal{A}_{s t}^{P, i, *}-\mathcal{A}_{s t}^{N, i, *}\right) \phi\right)\right| \\
& \lesssim_{N_{0}}\left|u_{s}^{N}-u_{s}\right|_{0}|\phi|_{2}+\left|u_{s}\right|_{0}\left|\left(\mathcal{A}_{s t}^{P, i, *}-\mathcal{A}_{s t}^{N, i, *}\right) \phi\right|_{0} \rightarrow 0
\end{aligned}
$$

as $N \rightarrow \infty$. Finally, using the strong convergence in $L_{T^{*}}^{2} \mathbf{H}^{1}$ of $\left\{u^{N}\right\}$ and (2.4) and the convergence of $P_{N}$ to the identity, we find

$$
\begin{aligned}
& \mid \int_{s}^{t} {\left[B_{P}\left(u_{r}\right)(\phi)-P_{N} B_{P}\left(u_{r}^{N}\right)(\phi)\right] d r \mid } \\
& \leq \int_{s}^{t}\left|B_{P}\left(u_{r}-u_{r}^{N}, u_{r}\right)(\phi)\right| d r+\int_{s}^{t}\left|B_{P}\left(u_{r}^{N}, u_{r}-u_{r}^{N}\right)(\phi)\right| d r \\
& \quad+\int_{s}^{t}\left|B_{P}\left(u_{r}^{N}\right)\left(\left(I-P_{N}\right) \phi\right)\right| d r \rightarrow 0
\end{aligned}
$$

as $N \rightarrow \infty$. 
Since all of the terms in equation (6.5) converge when applied to $\phi \in \mathbf{H}^{2}$, the remainder $u_{s t}^{N, \natural}(\phi)$ converges to some limit $u_{s t}^{\natural}(\phi)$. By virtue of (6.15), we obtain that the limit $u^{\natural} \in$ $C_{2, \omega_{Z}, L}^{\frac{p}{3} \text {-var }}\left(\left[0, T^{*}\right] ; \mathbf{H}^{-2}\right)$ for some positive constant $L=L\left(p,|\sigma|_{3, \infty}\right)>0$, which proves that $u$ is a strong solution to (3.1).

\section{APPENDIX: ROUGH GRONWALL LEMMA}

In this section, we formulate two Gronwall inequalities involving controls. The first one is a slight generalization of the Gronwall inequalities proved in [12] and [18], and can be proved by the same reasoning. The second inequality is a corollary of the first inequality and the classical Bihari-LaSalle inequality.

LEMmA A.2. Assume that $G:[0, T] \rightarrow \mathbf{R}_{+}$is such that there exists constants $L>0$ and $\kappa>0$, and a control $\omega$ such that for every $(s, t) \in \Delta_{T}$ with $\omega(s, t) \leq L$,

$$
\delta G_{s t} \leq \omega(s, t)^{\frac{1}{\kappa}} \sup _{0 \leq r \leq t} G_{t}+\phi(s, t)
$$

where $\phi: \Delta_{T} \rightarrow \mathbf{R}_{+}$is such that $\phi(s, t) \leq \phi(0, T)$. Then there exists a constant $K>0$ depending only on $\omega$ such that

$$
\sup _{0 \leq t \leq T} G_{t} \leq 2 \exp \left\{\frac{\omega(0, T)}{L \alpha}\right\}\left(G_{0}+K \phi(0, T)\right),
$$

where $\alpha:=1 \vee L^{-1}\left(2 e^{2}\right)^{-\kappa}$.

REMARK A.3. The only difference between this version and the version in [12] is that we do not require $\phi$ to be a control, or even superadditive, as in [18].

LEMMA A.4. Assume that $w: \mathbf{R}_{+} \rightarrow \mathbf{R}_{+}$is a nondecreasing continuous function with $w>0$ on $(0, \infty)$. Moreover, assume that $G:[0, T] \rightarrow \mathbf{R}_{+}$is such that there exists constants $L>0$ and $\kappa>0$, and a control $\omega$ such that for every $(s, t) \in \Delta_{T}$ with $\omega(s, t) \leq L$, we have

$$
\delta G_{s t} \leq C \int_{s}^{t} w\left(\sup _{0 \leq r^{\prime} \leq r} G_{r^{\prime}}\right) d r+\omega(s, t)^{\frac{1}{\kappa}} \sup _{0 \leq r \leq t} G_{r} .
$$

Then there exists a constant $K>0$ depending only on $\omega$ such that

$$
\sup _{0 \leq t \leq T^{*}} G_{t} \leq W^{-1}\left(W\left(q G_{0}\right)+T^{*} C K q\right),
$$

where

$$
q:=2 \exp \left\{\frac{\omega(0, T)}{L \alpha}\right\}, \quad \alpha:=1 \vee L^{-1}\left(2 e^{2}\right)^{-\kappa},
$$

$W$ is chosen such that $W^{\prime}(x)=(w(x))^{-1}$, and $T^{*}>0$ is such that

$$
W\left(q G_{0}\right)+T^{*} C K q \in \operatorname{Dom}\left(W^{-1}\right) .
$$

Proof. Define $\phi(s, t)=C \int_{s}^{t} w\left(\sup _{0 \leq r^{\prime} \leq r} G_{r^{\prime}}\right) d r$. By Lemma A.2 we obtain

$$
\sup _{0 \leq r \leq t} G_{r} \leq q G_{0}+q C K \int_{0}^{t} w\left(\sup _{0 \leq r^{\prime} \leq r} G_{r^{\prime}}\right) d r \leq q G_{0}+q C K \int_{0}^{t} w\left(\sup _{0 \leq r^{\prime} \leq r} G_{r^{\prime}}\right) d r,
$$

and the result now follows from the classical Bihari-LaSalle inequality. 
Acknowledgments. We are enormously grateful for helpful and inspiring discussions with Dan Crisan, Darryl Holm, Peter Friz and Remigijus Mikulevicius.

Moreover, we would like to thank the anonymous referees, whose comments and corrections helped improve the readability and quality of the paper.

The first and third authors were supported by the DFG via Research Unit FOR 2402.

\section{REFERENCES}

[1] Abraham, R., Marsden, J. E. and Ratiu, T. (2012). Manifolds, Tensor Analysis, and Applications, 2nd ed. Applied Mathematical Sciences 75. Springer, New York. MR0960687 https://doi.org/10.1007/ 978-1-4612-1029-0

[2] Bailleul, I. and Gubinelli, M. (2017). Unbounded rough drivers. Ann. Fac. Sci. Toulouse Math. (6) 26 795-830. MR3746643 https://doi.org/10.5802/afst.1553

[3] Bailleul, I., Riedel, S. and Scheutzow, M. (2017). Random dynamical systems, rough paths and rough flows. J. Differential Equations 262 5792-5823. MR3624539 https://doi.org/10.1016/j.jde.2017. 02.014

[4] BesSe, N. and FrISCH, U. (2017). Geometric formulation of the Cauchy invariants for incompressible Euler flow in flat and curved spaces. J. Fluid Mech. 825 412-478. MR3692802 https://doi.org/10. $1017 / \mathrm{jfm} .2017 .402$

[5] Brzeźniak, Z., CAPIŃski, M. and Flandoli, F. (1991). Stochastic partial differential equations and turbulence. Math. Models Methods Appl. Sci. 1 41-59. MR1105007 https://doi.org/10.1142/ S0218202591000046

[6] BrZeźniak, Z., CAPiński, M. and Flandoli, F. (1992). Stochastic Navier-Stokes equations with multiplicative noise. Stoch. Anal. Appl. 10 523-532. MR1185046 https://doi.org/10.1080/ 07362999208809288

[7] Brzeźniak, Z., Flandoli, F. and Maurelli, M. (2016). Existence and uniqueness for stochastic 2D Euler flows with bounded vorticity. Arch. Ration. Mech. Anal. 221 107-142. MR3483892 https://doi.org/10.1007/s00205-015-0957-8

[8] Cotter, C., Crisan, D., Holm, D. D., Pan, W. and Shevchenko, I. (2019). Numerically modeling stochastic Lie transport in fluid dynamics. Multiscale Model. Simul. 17 192-232. MR3904409 https://doi.org/10.1137/18M1167929

[9] Cotter, C. J., GotTwald, G. A. and Holm, D. D. (2017). Stochastic partial differential fluid equations as a diffusive limit of deterministic Lagrangian multi-time dynamics. Proc. R. Soc. Lond. Ser. A Math. Phys. Eng. Sci. 473 20170388, 10. MR3710332 https://doi.org/10.1098/rspa.2017.0388

[10] Crisan, D., Flandoli, F. and Holm, D. D. (2019). Solution properties of a 3D stochastic Euler fluid equation. J. Nonlinear Sci. 29 813-870. MR3948949 https://doi.org/10.1007/s00332-018-9506-6

[11] DAviE, A. M. (2008). Differential equations driven by rough paths: An approach via discrete approximation. Appl. Math. Res. Express. AMRX 2008 Art. ID abm009, 40. MR2387018

[12] Deya, A., Gubinelli, M., HofmanovÁ, M. and Tindel, S. (2019). A priori estimates for rough PDEs with application to rough conservation laws. J. Funct. Anal. 276 3577-3645. MR3957994 https://doi.org/10.1016/j.jfa.2019.03.008

[13] DiPerna, R. J. and Lions, P.-L. (1989). Ordinary differential equations, transport theory and Sobolev spaces. Invent. Math. 98 511-547. MR1022305 https://doi.org/10.1007/BF01393835

[14] Faranda, D., Pons, F. M. E., Dubrulle, B., Daviaud, F., Saint-Michel, B., Herbert, É. and CORTET, P.-P. (2014). Modelling and analysis of turbulent datasets using auto regressive moving average processes. Phys. Fluids 26105101.

[15] Flandoli, F. and GatareK, D. (1995). Martingale and stationary solutions for stochastic NavierStokes equations. Probab. Theory Related Fields 102 367-391. MR1339739 https://doi.org/10.1007/ BF01192467

[16] Friz, P. K. and Hairer, M. (2014). A Course on Rough Paths: With an Introduction to Regularity Structures. Universitext. Springer, Cham. MR3289027 https://doi.org/10.1007/978-3-319-08332-2

[17] FrIZ, P. K. and VICTOIR, N. B. (2010). Multidimensional Stochastic Processes as Rough Paths: Theory and Applications. Cambridge Studies in Advanced Mathematics 120. Cambridge Univ. Press, Cambridge. MR2604669 https://doi.org/10.1017/CBO9780511845079

[18] Hocquet, A. and Hofmanová, M. (2018). An energy method for rough partial differential equations. J. Differential Equations 265 1407-1466. MR3797622 https://doi.org/10.1016/j.jde.2018.04.006

[19] Hocquet, A. and Nilssen, T. (2021). An Itô formula for rough partial differential equations. Application to the maximum principle. Potential Anal. 54 331-386. MR4202743 https://doi.org/doi.org/10.1007/ s11118-020-09830-y 
[20] Hocquet, A., Nilssen, T. and Stannat, W. (2020). Generalized Burgers equation with rough transport noise. Stochastic Process. Appl. 130 2159-2184. MR4074697 https://doi.org/10.1016/j.spa.2019.06. 014

[21] Hofmanová, M., Leahy, J.-M. and Nilssen, T. (2019). On the Navier-Stokes equation perturbed by rough transport noise. J. Evol. Equ. 19 203-247. MR3918521 https://doi.org/10.1007/ s00028-018-0473-z

[22] Holm, D. D. (2015). Variational principles for stochastic fluid dynamics. Proc. R. Soc. Lond. Ser. A Math. Phys. Eng. Sci. 471 20140963, 19. MR3325187 https://doi.org/10.1098/rspa.2014.0963

[23] Lilly, J. M., SYKulski, A. M., EARly, J. J., Olhede, S. C. and MAY, M. E. (2017). Fractional Brownian motion, the Matérn process, and stochastic modeling of turbulent dispersion. Nonlinear Process. Geophys. 24 481-514.

[24] LIU, W. and RöCKNeR, M. (2015). Stochastic Partial Differential Equations: An Introduction. Universitext. Springer, Cham. MR3410409 https://doi.org/10.1007/978-3-319-22354-4

[25] Lyons, T. J., Caruana, M. and LÉvy, T. (2007). Differential Equations Driven by Rough Paths. Lecture Notes in Math. 1908. Springer, Berlin. MR2314753

[26] Majda, A. J., Timofeyev, I. and Vanden-Eijnden, E. (2003). Systematic strategies for stochastic mode reduction in climate. J. Atmos. Sci. 60 1705-1722. MR2030132 https://doi.org/10.1175/ 1520-0469(2003)060<1705:SSFSMR >2.0.CO;2

[27] Mikulevicius, R. (2002). On the Cauchy problem for stochastic Stokes equations. SIAM J. Math. Anal. 34 121-141. MR1950829 https://doi.org/10.1137/S0036141001390312

[28] Mikulevicius, R. and Rozovskit, B. L. (2004). Stochastic Navier-Stokes equations for turbulent flows. SIAM J. Math. Anal. 35 1250-1310. MR2050201 https://doi.org/10.1137/S0036141002409167

[29] Mikulevicius, R. and RozovskiI, B. L. (2005). Global $L_{2}$-solutions of stochastic Navier-Stokes equations. Ann. Probab. 33 137-176. MR2118862 https://doi.org/10.1214/009117904000000630

[30] TAO, T. (2016). Finite time blowup for Lagrangian modifications of the three-dimensional Euler equation. Ann. Partial Differ. Equ. 2 Art. 9, 79. MR3595455 https://doi.org/10.1007/s40818-016-0019-z

[31] TaYlor, M. E. (2011). Partial Differential Equations III. Nonlinear Equations, 2nd ed. Applied Mathematical Sciences 117. Springer, New York. MR2744149 https://doi.org/10.1007/978-1-4419-7049-7

[32] Temam, R. (1983). Navier-Stokes Equations and Nonlinear Functional Analysis. CBMS-NSF Regional Conference Series in Applied Mathematics 41. SIAM, Philadelphia, PA. MR0764933

[33] ZHU, R. and ZHU, X. (2015). Three-dimensional Navier-Stokes equations driven by space-time white noise. J. Differential Equations 259 4443-4508. MR3373412 https://doi.org/10.1016/j.jde.2015.06. 002 